\title{
Experimental and Numerical Investigations of Confluent Round Jets
}

Klas Svensson

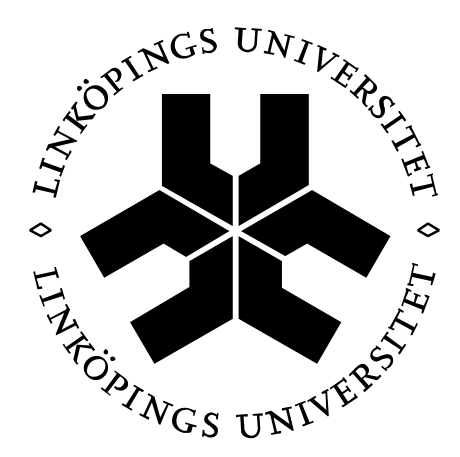

\section{Linköping University}

INSTITUTE OF TECHNOLOGY

Division of Energy Systems

Department of Management and Engineering

Linköping University, Sweden

Linköping 2015 
Experimental and Numerical Investigations of Confluent Round Jets

(C)Klas Svensson, 2015

Printed in Sweden by LiU-Tryck, Linköping, Sweden, 2015

ISBN: 978-91-7519-086-0

ISSN: 0345-7524 


\section{Abstract}

Unconfined multiple interacting confluent round jets are interesting from a purely scientific point of view, as interaction between neighboring jets brings additional complexity to the flow field. Unconfined confluent round jets also exist in various engineering applications, such as ventilation supply devices, sewage disposal systems, combustion burners, chemical mixing or chimney stacks. Even so, little scientific attention has been paid to unconfined confluent round jets, and detailed investigations on the complete three-dimensional turbulent flow field of confluent jet configurations issuing into an unconfined stagnant environment are scarce.

The present work uses both advanced measurement techniques and computational models to provide deeper understanding of the turbulent flow field development of unconfined confluent round jets. Both Laser Doppler Anemometry (LDA) and Particle Image Velocimetry (PIV) have been used to measure mean velocity and turbulence properties within two setups, consisting of a single row of $1 \times 6$ jets and a square in-line array of $6 \times 6$ confluent jets. LDA was used for measurements very close to the nozzle inlets and to verify the main findings made with PIV. A comparison between LDA and PIV results showed that the two measurement techniques produced results that are in good agreement with each other.

Simulations using Computational Fluid Dynamics (CFD) of the $6 \times 6$ setup were conducted using three different Reynolds Averaged Navier-Stokes (RANS) turbulence models: the standard $k-\varepsilon$, the RNG $k-\varepsilon$ and the Reynolds Stress Model (RSM). The results from the CFD simulations were compared with the experimental PIV data. The employed RANS turbulence models were all capable of accurately predicting mean velocities and turbulent properties in the investigated confluent jet array. In general the RSM and the standard $k-\varepsilon$ model provided smaller deviations between numerical and experimental results than the RNG $k-\varepsilon$ model. In terms of mean velocity the second-order closure model (RSM) was not found to be superior to the less complex standard $k-\varepsilon$ model.

The validated CFD results provide data at all locations in the solution domain and the computational results were used to further analyze the investigated confluent jet array. The validated CFD model was also employed in a parametrical investigation, including five independent variables: inlet velocity, nozzle diameter, nozzle edge-toedge spacing, nozzle height and the number of jets in the array. The parametrical investigations made use of statistical methods in the form of Response Surface Methodology and Box-Behnken Design of Experiments. The derived response surface models provided information on the principal influence and relative importance of the investigated parameters within the design limits. 
The positions of the jets within the array strongly influence both mean velocity and turbulence. In all investigated setups the jets experience merging and combining. Square arrays also include considerable jet convergence, which was not present in the $1 \times 6$ jet array. Due to the jet convergence in square arrays the turbulent flow field, especially for jets far away from the array center, is affected by mean flow curvature.

Jets located along the sides of square jet arrays experience strong jet-to-jet interactions that result in considerable jet deformation, shorter potential core, higher turbulent kinetic energy and faster velocity decay compared to other jets. Jets located at the corners of the array do not interact as strongly with neighboring jets as do the jets along the sides. In twin or multiple jet configurations, locations of merging and combined points are important characteristics, as also in the investigated confluent round jet arrays. The locations of merging and combined points differ considerably between different jets and different jet configurations.

As the jets combine a zone with uniform stream-wise velocity and low turbulence intensity forms in the center of square jet arrays. This zone has been called Confluent Core Zone (CCZ) due to its similarities with the potential core zone of a single jet. Within the CCZ the appropriate scaling length changes from nozzle diameter to the effective source diameter.

The parametrical investigation showed that nozzle diameter and edge-to-edge nozzle spacing were the most important of the investigated parameters, reflecting a strong dependence on dimensionless jet spacing, $S / d_{0}$. Higher $S / d_{0}$ delays both merging and combining of the jets and leads to a CCZ with lower velocity and longer downstream extension. Increasing the array size leads to a reduced combined point distance, stronger sub-atmospheric pressure between the jets and a stronger inwards displacement of jets in the outer part of the array. Larger array size also extends the CCZ, reduces the turbulence intensity in the CCZ and reduces the entrainment near the nozzles. A higher inlet velocity was found to increase the jet convergence in the investigated square confluent jet arrays. Nozzle height generally has minor impact on the investigated response variables. 


\section{Sammanfattning}

Matriser av interagerande och samverkande runda jetstrålar, benämnda som confluent jets, är intressanta ur ett rent vetenskapligt perspektiv, eftersom interaktioner mellan angränsande strålar, bidrar till ett förändrat och mer komplext flödesfält. Friströmmande samverkande jetstrålar har också en rad olika tekniska tillämpningar, såsom tilluftsdon $\mathrm{i}$ ventilationssystem, system för bortföring av avloppsvatten, förbränningskammare, omrörning eller blandning av flytande eller gasformiga material, eller industriella skorstenar. Detta till trots har friströmmande samverkande jetstrålar hittills endast fått begränsad vetenskaplig uppmärksamhet och bara ett fåtal detaljerade undersökningar av det turbulenta flödesfältet hos den typen av jetkonfiguration finns rapporterade.

I denna studie används både avancerade mättekniker och numeriska modeller för att skapa en djupare förståelse för utvecklingen av det turbulenta hastighetsfältet hos friströmmande samverkande jetstrålar. Laser Doppler Anemometri (LDA) och Particle Image Velocimetry (PIV) har använts för att mäta medelhastigheter och turbulens $\mathrm{i}$ två olika jetkonfigurationer. De två uppställningarna som undersökts experimentellt består av en enda rad av $1 \times 6$ strålar och en matris med $6 \times 6$ strålar. LDA används för mätningar mycket nära inloppen och för att bekräfta viktiga aspekter uppmärksammade i PIV mätningarna. En jämförelse mellan LDA- och PIVresultat visade på god överenstämmelse mellan de två mätteknikerna.

Numeriska simuleringar, i form av Computational Fluid Dynamics (CFD), utfördes för uppställningen med $6 \times 6$ samverkande jetstrålar. CFD-simuleringarna genomfördes med tre olika turbulensmodeller; standard $k-\varepsilon$, RNG $k-\varepsilon$ och Reynolds Stress Model (RSM). Resultaten från CFD-simuleringarna jämfördes med mätdata från PIV-mätningar. Samtliga använda turbulensmodeller uppvisade god överenstämmelse med mätdata för den undersökta jetkonfigurationen. I allmänhet uppvisade CFD-simuleringar med RSM och standard $k-\varepsilon$ modellen mindre avvikelser jämfört med mätdata än RNG $k-\varepsilon$ modellen. Vad gäller prediktering av hastighetsfält visade sig RSM inte vara överlägsen den mindre komplicerade standard $k-\varepsilon$ modellen.

Den validerade CFD-modellen tillhandahåller information om strömningsbilden $\mathrm{i}$ samtliga positioner inom beräkningsdomänen. Inom detta arbete används därför de numeriska resultaten för att ytterligare analysera den aktuella konfigurationen av jetstrålar, utöver den experimentella analysen. Den validerade CFD-modellen användes också $\mathrm{i}$ en parameterstudie som inkluderade fem oberoende variabler: inloppshastighet, inloppsdiameter, kant-till-kantavstånd mellan olika inlopp, inloppsmunstyckets höjd och antalet jetstrålar i matrisen. 
Parameterstudien utfördes med hjälp statistiska metoder i form av Response Surface Methodology och Box-Behnken Design of Experiments. De resulterande funktionsytorna beskriver olika parametrars principiella inverkan samt deras relativa betydelse i jämförelse med varandra.

Strålens position inom matrisen visade sig ha stor inverkan på utvecklingen av både hastighetsfält och turbulens. I samtliga undersökta konfigurationer interagerar strålarna efter ett tag med varandra, och går sedan samman till en gemensam jetstråle. Kvadratiska matriser av $6 \times 6,8 \times 8$ eller $10 \times 10$ jetstrålar uppvisar också en betydande avböjning, eller konvergens, av strålarna mot matrisens centrum. Detta kunde inte observeras i den enkla raden av $1 \times 6$ jetstrålar. På grund av strålarnas avböjning mot centrum, kommer jetstrålarnas skjuvlager att påverkas av kurvaturen hos hastighetsfältet. Detta gäller i synnerhet jetstrålar med stort avstånd till matrisens centrum.

Jetstrålar placerade längs sidorna av kvadratiska matriser interagerar kraftigt med omgivande strålar. Detta resulterar i betydande deformation av jetstrålen, en kortare kärnzon, högre turbulent kinetisk energi och snabbare avklingning av maxhastigheten jämfört med andra jetstrålar. Jetstrålar belägna i hörnen av matrisen interagerar inte lika kraftigt med omgivande jetstrålar. I andra, mer grundläggande konfigurationer av interagerande jetsstålar, används positionerna där interaktionerna mellan strålar påbörjas och fullbordas, för att separera utvecklingen i olika regioner. Denna kategorisering kan användas även för jetstrålarna i denna studie. Resultaten här visar att positionen där interaktioner mellan jetstålar påbörjas och fullbordas skiljer sig avsevärt mellan olika positioner i matrisen och olika konfigurationer.

Efter att jetstrålarna hos kvadratiska matriser gått samman till en gemensam stråle uppstår en zon med en enhetlig hastighet i strömningsriktningen och låg turbulensintensitet. Denna zon har döpts till Confluent Core Zone (CCZ) på grund av dess likheter med kärnzonen hos en enskild stråle. Inom CCZ:n ändras lämplig skalparameter för längd från inloppsdiameter till den effektiva diameterna hos matrisen som helhet.

Parameterstudien visade att inloppsdiameter och kant-till-kantavståndet mellan inloppen är de mest betydelsefulla av de undersökta parametrarna, vilket återspeglar ett starkt beroende på det dimensionslösa avståndet mellan jetstrålar, $S / d_{0}$. Högre $S / d_{0}$ fördröjer både start och fullbordan av interaktioner mellan strålar och leder till en CCZ med lägre hastighet och längre nedströms förlängning. Ökad matrisstorlek leder till snabbare sammanslagning till en genomensam stråle, ett starkare undertryck mellan strålarna och en kraftigare avböjning mot matrisens centrum hos jetstrålarna i matrisenes utkanter. Större matrisstorlek förlänger också CCZ:n, minskar turbulensintensiteten i CCZ:n och minskar inträngningen av omgivande luft nära inloppen. En högre inloppshastighet visade sig öka jetstrålarnas avböjning mot matrisens centrum i de undersökta kvadratiska matriserna. Inloppsmunstyckenas höjd har i allmänhet föga inverkan på utvecklingen. 
Till Stina 



\section{Acknowledgements}

First of all I would like to thank my supervisor Prof. Bahram Moshfegh for giving me the opportunity to getting to know the world of research and for all the support over the years. I have really enjoined discussing both problems and opportunities with you. Your comments always take the work forward.

Moreover, I would like to say thank you to my co-supervisor Dr. Patrik Rohdin. Thank you for your patience all the hundreds of times I have knocked on your door with whatever questions on turbulence models, convergences problems, Fluent debugging, article writing or thesis structure.

I would like to thank the former $\mathrm{PhD}$ student Shahriar Ghahremanian for the fruitful co-operation during the experimental campaign and when writing the resulting papers. It has been a pleasure working with you. Congratulations to your degree!

Furthermore, I earn a great deal of gratefulness to Dr. Mark Tummers, Delft University of Technology, for all the guidance during the experimental work and for invaluable comments on the resulting papers. I would also like to send a special thank you to the laboratory personnel at Delft University of Technology, especially Mr. Bart Hoek and Mr. Erwin de Beus for their help with the experimental setup.

I would like to thank the Swedish Research Council and Linköping University for the financial support, the National Supercomputer Centre for providing computational resources and finally Stravent Oy (Finland) and Mr. Timo Karkulahti for taking the initiative to the project and providing prototypes for the experimental investigation.

Thank you to all my colleagues at the Division of Energy Systems for making my work days so much more fun, through all the fika-discussions on small and big topics, like politics or how to behave when exercising.

"Du kan ju àtminstone pröva ett halvår och se hur det går? Det är ju inte värre än att du slutar om du inte klarar de första tentorna". Tack mamma och pappa för att ni stod på er när jag tvivlade på att universitetsstudier var något för mig och för allt ert stöd genom åren. Ni har hjälpt mig med allt från flytt och studiebidrag till nattliga tur-och-retur-färder med bortglömda legitimationer. Ni har alltid varit så förstående mot mig. Tack.

Stina, mitt eviga stöd och blivande fru. Hur ska jag någonsin kunna återgälda dig? Att du fortfarande överhuvudtaget orkar lyssna på mig är ett mirakel. Ditt stöd den senaste tiden har varit ovärderligt. Jag hade inte klarat mig utan dig. Tack för att du vill dela ditt liv med mig. 



\section{Nomenclature}

Symbol

$A_{I}$

$A_{\text {inlet }}$

$b$

$C_{i j}$

$C_{1 \varepsilon}, C_{2 \varepsilon}$

$C_{\mu}$

$D_{i j, L}, D_{i j, T}$

$D_{i j, T}^{*}$

do

$d_{f}$

$d_{p}$

$d_{s}$

$d_{\tau}$

E

$F_{I}$

$F_{o}$

$f^{\#}$

$f_{D}$

$H$

K

k

$L$
Description

\section{Unit}

interrogation area

$\mathrm{m}^{2}$

total inlet area of jets

$\mathrm{m}^{2}$

jet spreading rate

convection term

$\mathrm{m}^{2} \mathrm{~s}^{-3}$

turbulence model coefficients

turbulent-viscosity constant

molecular and turbulent diffusion terms

$\mathrm{m}^{2} \mathrm{~s}^{-3}$

turbulent diffusion, including fluctuating pressure

$\mathrm{m}^{2} \mathrm{~s}^{-3}$

nozzle diameter

$\mathrm{m}$

fringe spacing

$\mathrm{m}$

tracer particle diameter

$\mathrm{m}$

effective source diameter, $d_{s}=\sqrt{4 A_{\text {inlet }} / \pi}$,

$\mathrm{m}$

or diffraction-limited spot diameter (Chapter 3)

particle image diameter

$\mathrm{m}$

Nozzle edge-to-edge spacing

$\mathrm{m}$

in-plane loss of correlation

out-of-plane loss of correlation

lens relative aperture ( $f^{\#}$-number)

measured Doppler frequency

$\mathrm{s}^{-1}$

height of computational domain

$\mathrm{m}$

velocity decay constant

turbulent kinetic energy or

$\mathrm{m}^{2} \mathrm{~s}^{-2}$

number of factors (Chapter 5)

length of computational domain

$\mathrm{m}$

turbulent length scale $\mathrm{m}$ 


\begin{tabular}{|c|c|c|}
\hline$M_{0}$ & image magnification factor & - \\
\hline$N_{I}$ & image density & - \\
\hline$P_{i j}$ & production term & $\mathrm{m}^{2} \mathrm{~s}$ \\
\hline$p, P, p^{\prime}$ & instantaneous, mean and fluctuating static pressure & $\mathrm{Pa}$ \\
\hline Q & volumetric flow rate & $\mathrm{m}^{3} \mathrm{~s}$ \\
\hline$Q_{0}$ & volumetric flow rate, inlet & $\mathrm{m}^{3} \mathrm{~s}-$ \\
\hline$Q_{c p, a}$ & $\begin{array}{l}\text { volumetric flow rate of jets at adjusted } \\
\text { combined point }\end{array}$ & $\mathrm{m}^{3} \mathrm{~s}^{-}$ \\
\hline$Q_{i}$ & $y$-axis interceptor, flow rate of combined jet & $\mathrm{m}^{3} \mathrm{~s}$ \\
\hline$R_{D}$ & displacement correlation peak & $\mathrm{J}^{2} \mathrm{~m}^{-}$ \\
\hline$R e_{d}, R e_{w}$ & $\begin{array}{l}\text { Reynolds number, based on nozzle diameter or } \\
\text { slot width }\end{array}$ & - \\
\hline$r_{0.5}$ & jet half-width & $\mathrm{m}$ \\
\hline$S$ & nozzle center-to-center spacing & $\mathrm{m}$ \\
\hline$S_{i j}$ & rate of strain tensor & $\mathrm{s}^{-1}$ \\
\hline$\Delta t$ & time delay, PIV exposures & $\mathrm{s}$ \\
\hline$T_{U}$ & $\begin{array}{l}\text { turbulence intensity in stream-wise direction, } \\
T_{U}=\frac{\sqrt{2 / 3 k}}{|U|}\end{array}$ & - \\
\hline$\overline{u_{i}^{\prime} u_{j}^{\prime}}$ & Reynolds stresses component (Chapter 4) & $\mathrm{m}^{2} \mathrm{~s}$ \\
\hline$\overline{u^{\prime 2}}, \overline{v^{\prime 2}}, \overline{w^{\prime 2}}$ & normal Reynolds stresses & $\mathrm{m}^{2} \mathrm{~s}$ \\
\hline$\overline{u^{\prime} v^{\prime}}, \overline{u^{\prime} w^{\prime}}, \overline{v^{\prime} w^{\prime}}$ & shear Reynolds stresses & $\mathrm{m}^{2} \mathrm{~s}^{-}$ \\
\hline$U, u^{\prime}$ & $\begin{array}{l}\text { mean and fluctuating velocity component, } \\
\text { stream-wise direction }\end{array}$ & $\mathrm{ms}^{-1}$ \\
\hline$U_{b}$ & inlet bulk velocity, $U_{b}=Q_{0} / A_{\text {inlet }}$ & $\mathrm{ms}^{-1}$ \\
\hline$U_{c l}$ & stream-wise velocity at jet geometrical centerline & $\mathrm{ms}^{-1}$ \\
\hline$U_{i}$ & mean velocity component in $x_{i}$ direction & $\mathrm{ms}^{-1}$ \\
\hline$U_{m}$ & overall maximum stream-wise velocity of all jets & $\mathrm{ms}^{-1}$ \\
\hline$U_{\max }$ & maximum stream-wise velocity of all jets & $\mathrm{ms}^{-1}$ \\
\hline$U_{\max , J}, U_{\min , J}$ & $\begin{array}{l}\text { maximum and minimum stream-wise velocity of } \\
\text { individual jet }\end{array}$ & $\mathrm{ms}^{-1}$ \\
\hline$U_{\max , \mathrm{Z}}$ & $\begin{array}{l}\text { maximum stream-wise velocity in } \\
\text { PIV measurement plane ( } z=\text { constant) }\end{array}$ & $\mathrm{ms}^{-1}$ \\
\hline
\end{tabular}




\begin{tabular}{|c|c|c|}
\hline$V, W, v^{\prime}, w^{\prime}$ & $\begin{array}{l}\text { mean and fluctuating velocity components, } \\
\text { span-wise (horizontal and vertical) directions }\end{array}$ & $\mathrm{ms}^{-1}$ \\
\hline$x$ & stream-wise coordinate & $\mathrm{m}$ \\
\hline$x_{0}, x_{0, b}$ & virtual origin & $\mathrm{m}$ \\
\hline$x_{m p}, x_{c p}, x_{c p, a}$ & $\begin{array}{l}\text { merging, combined, and adjusted combined } \\
\text { point distance }\end{array}$ & $\mathrm{m}$ \\
\hline$x_{i}=(x, y, z)$ & Cartesian coordinates & $\mathrm{m}$ \\
\hline$(\triangle X, \triangle Y)$ & particle displacement vector in image plane & $\mathrm{m}$ \\
\hline$(\triangle \tilde{x}, \triangle \tilde{y})$ & particle displacement vector in light sheet plane & $\mathrm{m}$ \\
\hline$y, z$ & span-wise (horizontal and vertical) coordinates & $\mathrm{m}$ \\
\hline$y_{c}, z_{c}$ & $\begin{array}{l}\text { coordinates jet geometrical centerline (horizontal } \\
\text { and vertical) }\end{array}$ & $\mathrm{m}$ \\
\hline$y^{\prime}, z^{\prime}$ & $\begin{array}{l}\text { local span-wise coordinate from jet } \\
\text { geometrical center line, }\left[y^{\prime}, z^{\prime}\right]=[y, z]-\left[y_{c}, z_{c}\right]\end{array}$ & $\mathrm{m}$ \\
\hline $\boldsymbol{X}$ & image plane coordinate system, PIV measurements & $\mathrm{m}$ \\
\hline$y^{+}, y^{*}$ & dimensionless distance from wall & - \\
\hline$w$ & slot width, plane twin jets & $\mathrm{m}$ \\
\hline$\triangle \tilde{z}_{0}$ & light sheet thickness & $\mathrm{m}$ \\
\hline \multicolumn{3}{|l|}{ Greeks } \\
\hline$\alpha, \alpha_{k}, \alpha_{\varepsilon}$ & inverse effective turbulent Prandtl number & - \\
\hline$\delta_{i j}$ & Kronecker delta & - \\
\hline$\varepsilon$ & rate of dissipation of turbulent kinetic energy & $\mathrm{m}^{2} \mathrm{~s}^{-3}$ \\
\hline$\varepsilon_{i j}$ & dissipation tensor & $m^{2} s^{-3}$ \\
\hline$\lambda$ & wave length, laser light & $\mathrm{m}$ \\
\hline$\mu$ & fluid dynamic viscosity & $\mathrm{kg} \mathrm{m}^{-1} \mathrm{~s}^{-1}$ \\
\hline$\Pi_{i j}$ & pressure-velocity gradient tensor & $\mathrm{m}^{2} \mathrm{~s}^{-3}$ \\
\hline$v$ & fluid kinematic viscosity & $\mathrm{m}^{2} \mathrm{~s}^{-1}$ \\
\hline$v_{t}$ & turbulent viscosity & $\mathrm{m}^{2} \mathrm{~s}^{-1}$ \\
\hline$\rho$ & fluid density & $\mathrm{kg} \mathrm{m}^{-3}$ \\
\hline$\sigma_{k}, \sigma_{\varepsilon}$ & turbulent Prandtl number & - \\
\hline$\Phi_{i j}, \Phi_{i j, 1}, \Phi_{i j, 2}, \Phi_{i j, w}$ & total, slow, rapid and wall pressure-strain terms & $m^{2} s^{-3}$ \\
\hline$\phi$ & conserved flow variable or scalar & \\
\hline
\end{tabular}


Abbreviations

BBD Box-Behnken Design

CCD Charged-Coupled Device or Central Composite Design (Chapter 5)

CCZ Confluent Core Zone

CFD Computational Fluid Dynamics

CJ Central Jet

CMOS Complementary Metal Oxide Semiconductor

CoJ Corner Jet

CVP Counter-Rotating Vortices Pair

FVM Finite Volume Method

LDA Laser Doppler Anemometry

PIV Particle Image Velocimetry

RS Response Surface

RSM Reynolds Stress Model

SJ Side Jet 


\section{Table of Contents}

1 Introduction 1

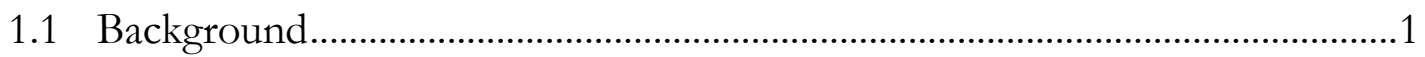

1.2 Motivation to the research .............................................................................

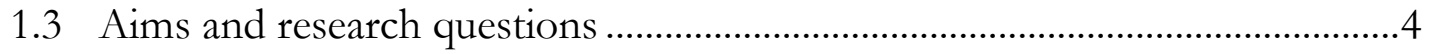

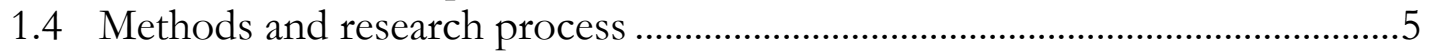

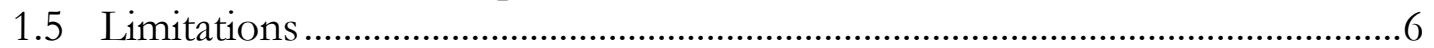

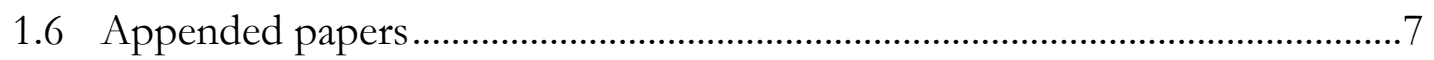

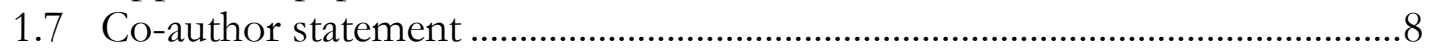

2 Theoretical background and literature review 9

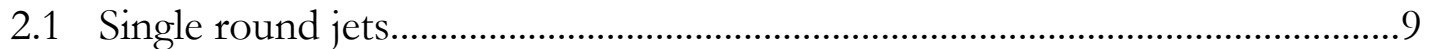

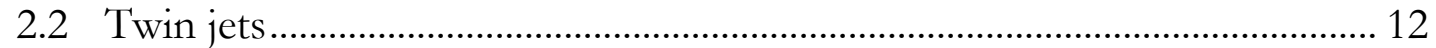

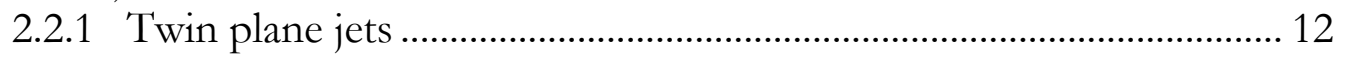

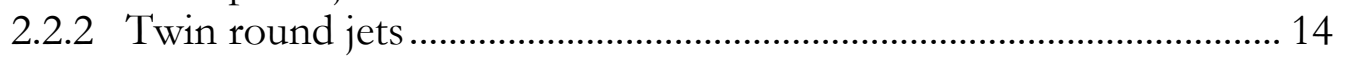

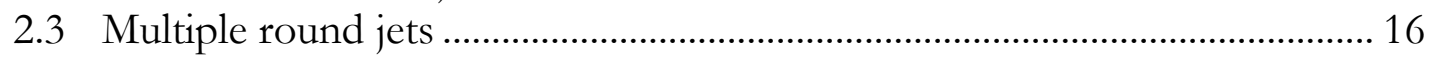

3 Measurement techniques 19

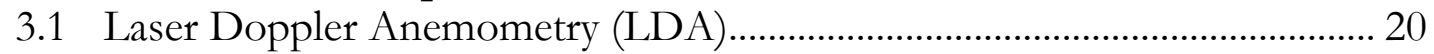

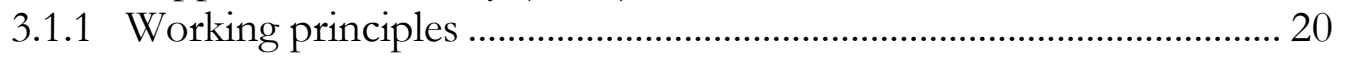

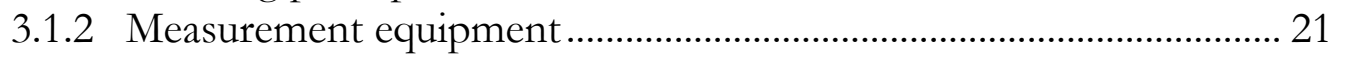

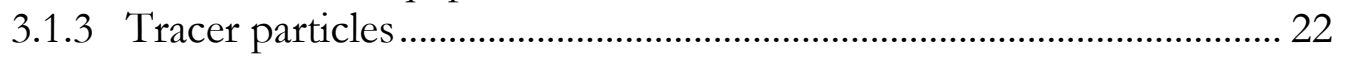

3.1.4 Post-processing and velocity bias ........................................................ 24

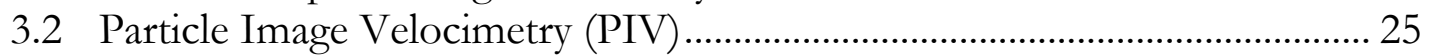

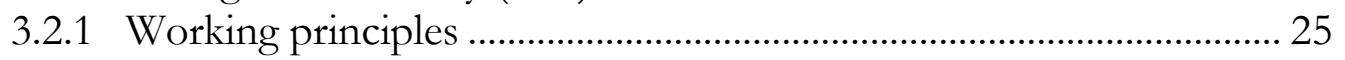

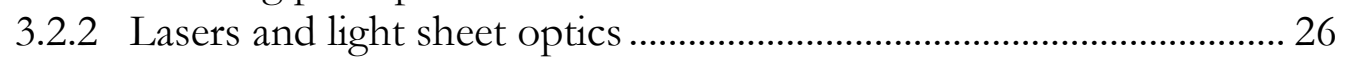

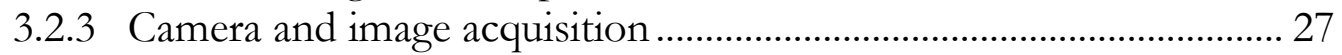

3.2.4 Vector calculation and post-processing .......................................... 29

3.3 Comparison of measurement techniques ....................................................... 33

4 Computational Fluid Dynamics $\mathbf{3 5}$

4.1 Turbulent flow and modeling strategies ......................................................... 36

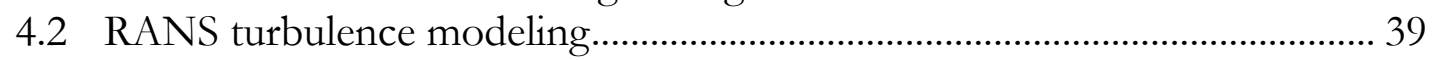

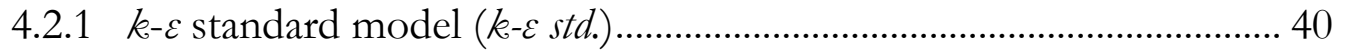

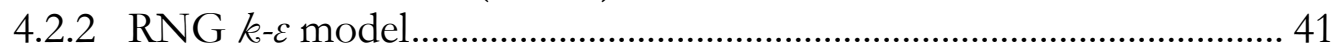

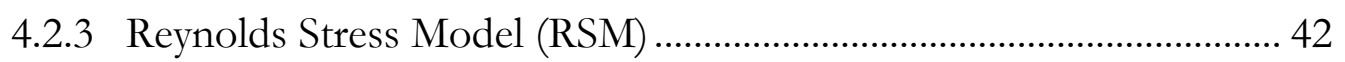

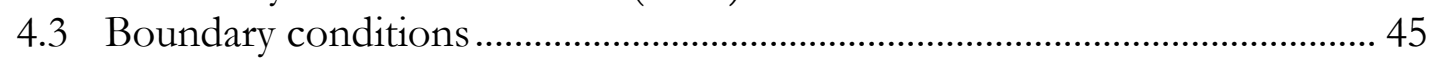


4.4 Numerical aspects.

$5 \quad$ Statistical methods $\quad 51$

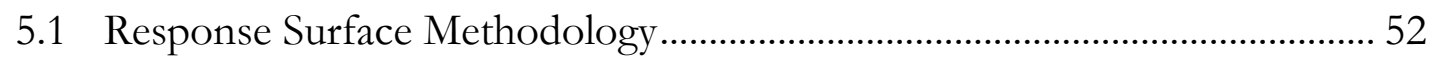

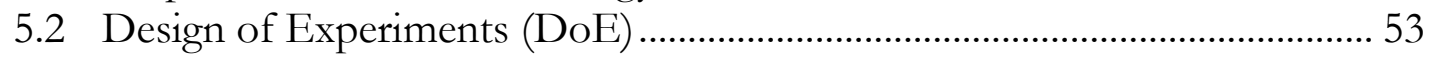

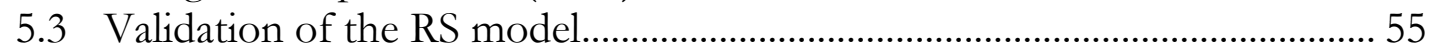

$6 \quad$ Experimental case studies $\quad 57$

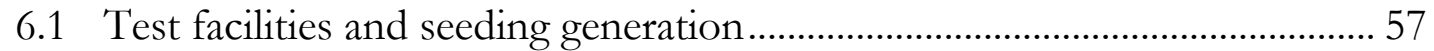

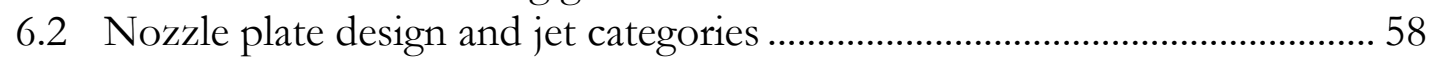

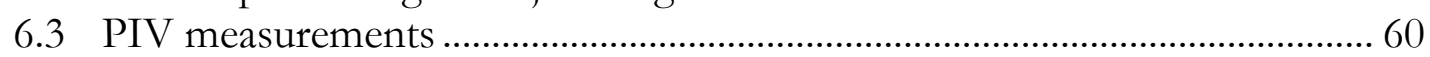

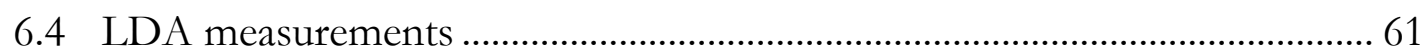

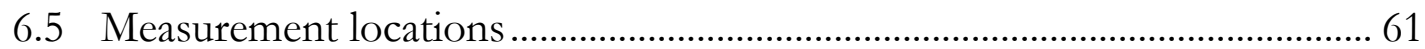

$7 \quad$ Computational model $\quad \mathbf{6 3}$

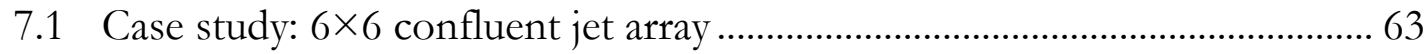

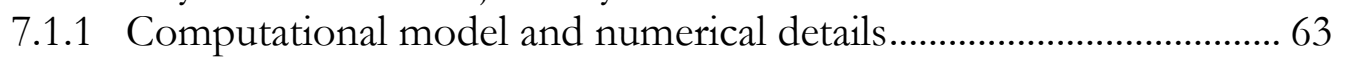

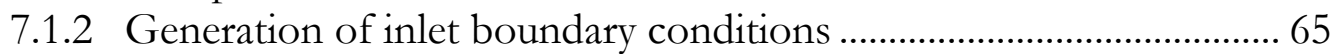

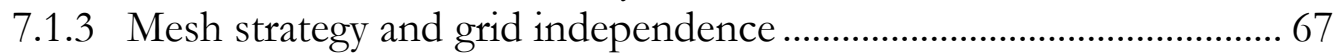

7.2 Parametrical investigation: CFD models and statistical approximation...... 68

8 Results $\quad 71$

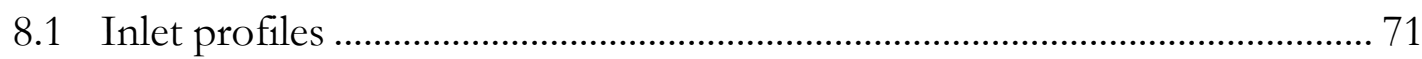

8.2 Comparison of measurement techniques .................................................... 72

8.3 Comparison between single row of confluent jets and square confluent

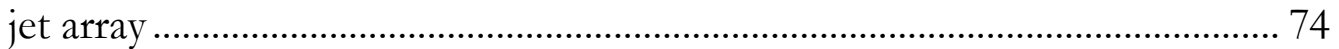

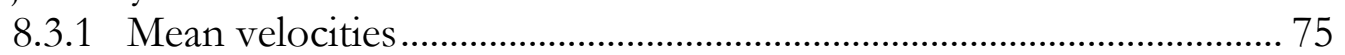

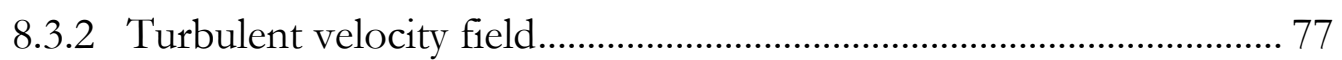

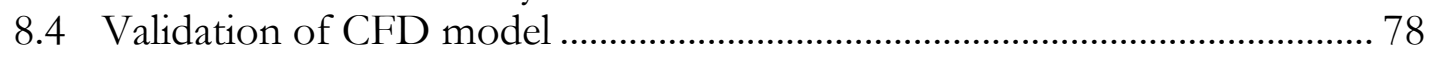

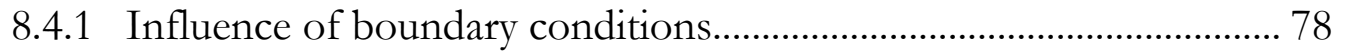

8.4.2 Influence of turbulence model ............................................................... 80

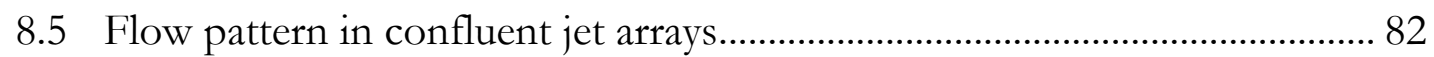

8.5.1 Development upstream of combined region..................................... 82

8.5.2 Combined jet development and Confluent Core Zone (CCZ) ........ 84

8.5.3 Characteristic points and regions of development............................. 88

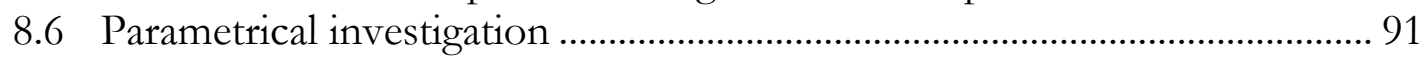

8.6.1 Accuracy of RS models ...................................................................... 92

8.6.2 Merging and combining characteristics............................................... 93

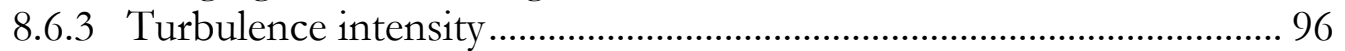

8.6.4 Confluent Core Zone and maximum velocity decay ........................... 97

8.6.5 Volumetric flow rate .............................................................................. 98

9 Conclusions $\quad 99$

$\begin{array}{lll}10 & \text { Future work } & 103\end{array}$

$\begin{array}{ll}\text { References } & 105\end{array}$ 


\section{Introduction}

This chapter starts with a brief background to the research, followed by the motivation of the work. Next, the aim of the study is presented and research questions are posed. Further on, the chapter brings a short discussion on research methods and limitations. The chapter ends with a list on appended papers and co-author statement.

\subsection{Background}

Turbulent jets in various configurations are one of the most scientifically investigated features of turbulent flow. They exist in nature, in the form of volcanoes, thermal plumes or where rivers exit into the ocean. Similar, they exist in numerous engineering applications, such as indoor ventilation, cooling towers, sewage disposal systems, chimney stacks, chemical mixing systems and combustion nozzles, to mention a few.

Consequently, as turbulent jets are interesting from both a purely scientific point of view and have numerous engineering applications, they have also been subject to extensive research. Scientific investigations on turbulent jets include mean flow properties, turbulent Reynolds stresses, scales of turbulence, turbulent production and anisotropy, dissipation or destruction of turbulence, mixing characteristics and vortex dynamics.

There are also a large number of different jet configurations to which scientific attention has been drawn. Scientific investigations on single turbulent jets include for example single free jets, impinging jets and wall jets. The jet shape may be either plane, axisymmetric or square shaped. Free jets may be considered as confined, partially unconfined or unconfined depending on the presence of solid objects. The jet may also be exposed to either co-flow or cross-flow. Research on turbulent jets includes both isothermal and non-isothermal jets.

Research on turbulent jets is not limited to single jets, as scientific work also includes multiple jets. Similar to single jets, multiple jets may have different inlet shapes, be confined or unconfined, and be considered free, impinging or wall jets. Multiple jets may also interact with each other, i.e., multiple interacting jets. Examples of interacting multiple jets are twin plane or twin round jets, but interacting multiple jets may also consist of a larger number of jets. Multiple interacting jets may also be exerted in the same direction, inclined, or directly opposed to each other. 
One example of multiple interacting jets is confluent jets, which consist of a number of closely spaced jets that issue in the same direction. After a certain downstream distance they will start to merge and eventually combine to a single jet (Awbi, 2003). The interaction between neighboring jets brings additional complexity to the flow field. The confluence will influence development of turbulence, mean velocity and volumetric flow rate. Therefore the development of turbulent confluent jets cannot be directly derived based on results from investigations on, for example, single or twin jets. Confluent jets exist in various applications. In Figure 1 two examples, a ventilation air supply device in an airport arrival hall, and a number of in-line industrial chimney stacks are shown to illustrate situations with multiple interacting confluent jets.

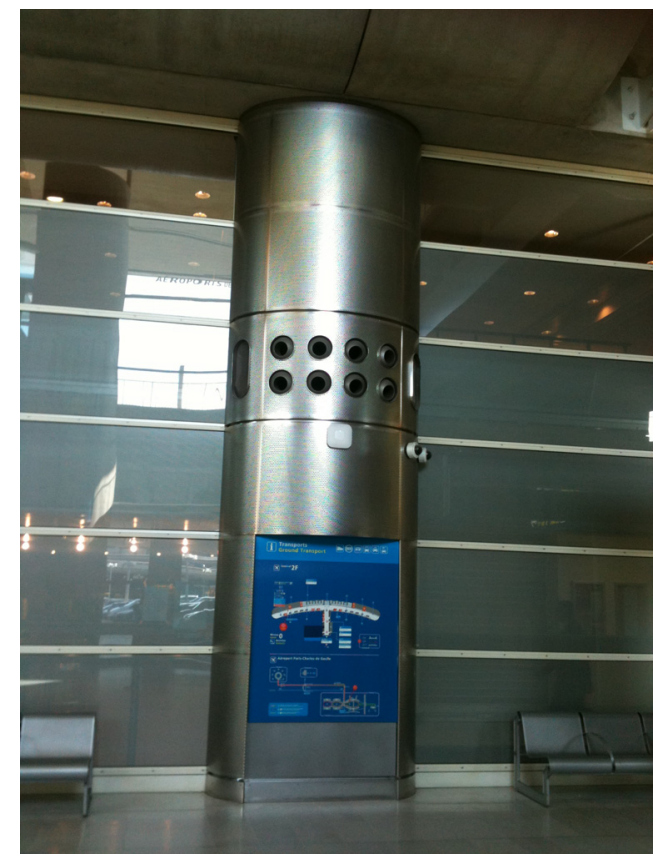

Ventilation supply device, airport terminal

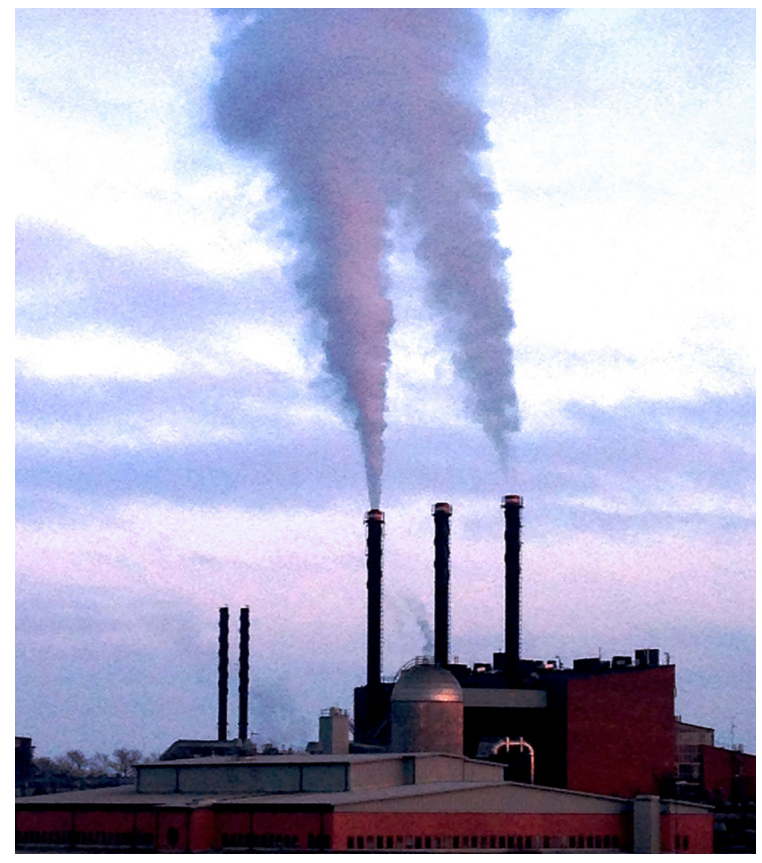

Industrial chimney stack, CHP plant in Linköping

Figure 1 Examples of confluent jets in engineering applications.

In recent decades, scientific tools for the investigation of turbulent flow features have undergone rapid development. Large advancements have been made in measurement techniques, and today e.g. by whole-field, directional sensitive measurements, deep insight can be gained in the turbulent behavior of fluids. The possibility to conduct accurate measurements in complex setups has led to an extension of experimental investigations to numerous industry-specific problems.

Perhaps even more scientific contributions to investigation of turbulent flow features are accomplished by the rise of advanced numerical methods in the form of Computational Fluid Dynamics (CFD). CFD has been made possible through the development of high performance computers, and has proven to be a leading scientific tool supported by limited experimental exploration.

The abovementioned development has provided tools for studying more complex jet configurations. Even so, little attention has been paid to unconfined multiple interacting confluent jets. 


\subsection{Motivation to the research}

There is a need for more detailed understanding and scientific description of unconfined confluent jets in various fields of engineering. A specific example is ventilation supply devices, which may be based on a number of confluent jets issuing from the same device. Shown in Figure 2 is the S11 supply device from Stravent Oy, which consists of a large number of confluent jets located on a cylinder. Research on thermal comfort and ventilation performance using confluent jet ventilation, both in an industrial and office environment, has been conducted by Janbakhsh et al. (2010) and Janbakhsh and Moshfegh (2014).

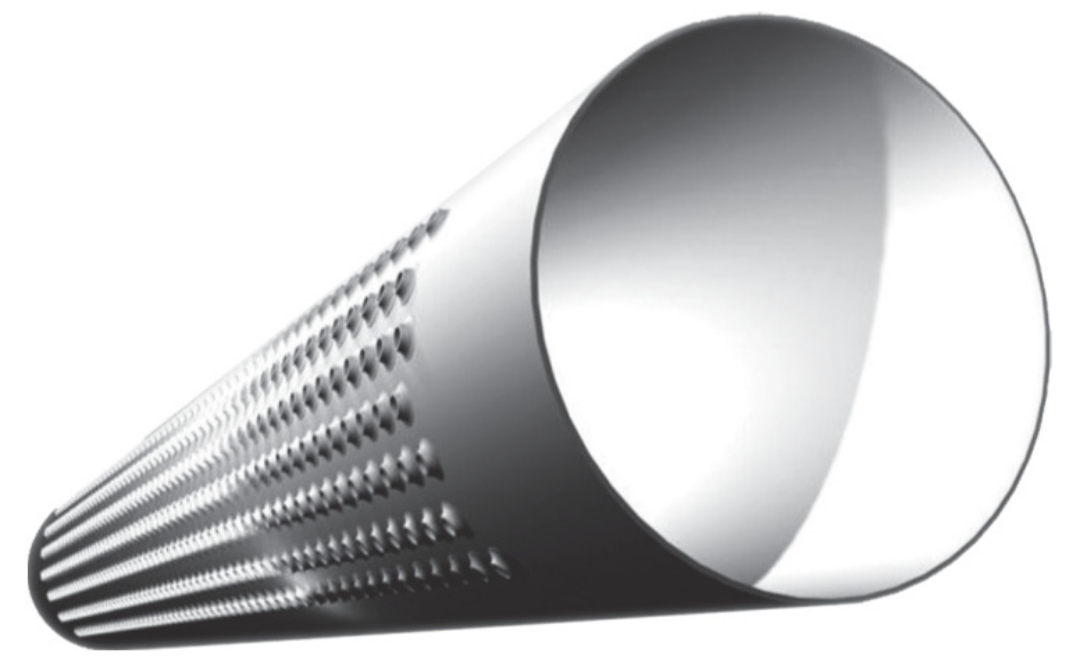

Figure 2 Ventilation supply device, model S11 by Stravent Oy (Stravent).

Especially the region close to the nozzles, where the jets transform from individual jets to a single combined jet through merging and combining, brings increasing complexity to the turbulent flow field. To be able to master the merging and combining of the confluent jets is a key to controlling the development of confluent jet arrays.

There are a number of contributions on multiple interacting jets, many of them conducted within the area of combustion research. However, previous studies mainly deal with what can be considered confined jets, meaning that their development is affected by the presence of side walls in the direction of the flow which limit or prevent entrainment of surrounding fluid. The investigations are also often conducted on custom-made arrangements.

Detailed investigations on complete three-dimensional, multiple interacting confluent jet configurations of a general character issuing into an unconfined stagnant environment, i.e, without being affected by solid side walls, are scarce. Therefore, little is known regarding arrays of unconfined confluent jets, for example in terms of jet-to-jet interactions, regions of development, entrainment of surrounding fluid and decay of maximum velocity. At the same time confluent jets that are practically unconfined are common and a scientific description that provides such knowledge is important for numerous engineering design problems. This includes optimization of ventilation performance, energy efficiency and thermal comfort for ventilation supply 
devices based on multiple jets, proper design of chimney stacks or waste water disposal systems that prevent human exposure to polluted air or water, optimization of chemical mixing and minimizing emissions in combustion systems based on confluent jets.

\subsection{Aims and research questions}

A deeper understanding of the turbulent flow field development of unconfined confluent round jets can be achieved by using both advance measurement techniques and CFD simulations. This is the primary target of the presented work. A secondary target, but still important, is the validation process of the three used steady-state RANS turbulence models, namely the standard $k-\varepsilon$ model, the RNG $k-\varepsilon$ model and the Reynolds Stress Model (RSM), applied to confluent jet configurations.

The present work aims to present important aspects of unconfined confluent round jets, such as development of mean velocity, turbulence and volumetric flow rate. Different regions of development are to be identified, and when feasible comparison with basic jet configurations, such as a single round jet, round or plane twin jets, or a single row of round jets will be conducted. Another objective of the work is to evaluate the performance of the abovementioned steady-state RANS turbulence models, through comparisons with experimental data, with the target of providing a validated computational model. Finally, in order to help design engineers master the development of confluent jets, the present work aims to provide information on the influence of different parameters on the development of unconfined confluent jets through parametrical investigations.

The above research aims may be formalized in the following research questions, around which the thesis is focused, for the studied cases.

Q1. What important aspects characterize the development of mean velocity, turbulent flow field, volumetric flow rate and jet merging and combining in different parts of the investigated confluent jet arrays, from the nozzle exit up to a maximum stream-wise distance of 150 inlet diameters $\left(d_{0}\right)$ ?

Q2. To what extent are the employed steady-state RANS turbulence models, i.e., standard $k-\varepsilon$, RNG $k-\varepsilon$ and RSM, capable of accurately predicting mean velocities and turbulence properties, such as Reynolds stresses, turbulent kinetic energy and turbulence intensity, of the investigated confluent jet array?

Q3. How do the parameters inlet velocity, nozzle diameter, nozzle spacing, nozzle beight and the number of jets (array size) influence mean velocity, regions of merging and combining, volumetric flow rate and development of turbulent flow field for the investigated unconfined confluent jets? 


\subsection{Methods and research process}

The present work employs a combination of experimental, computational (CFD) and statistical methods to investigate the development of unconfined confluent round jets arranged as a square in-line array or single row of jets. The research methods of the present investigations can be described in the following three steps:

\section{- Experimental study}

The experimental study makes use of two different advanced measurement techniques: Particle Image Velocimetry (PIV) and Laser Doppler Velocimetry (LDA). All measurements reported on in the present work are performed in a controlled lab environment at Department of Process and Energy, Faculty Mechanical, Maritime and Materials Engineering, Delft University of Technology, The Netherlands. The two experimental techniques are described in Chapter 3.

Two different confluent jet arrays, consisting of $6 \times 6$ and $1 \times 6$ jets were investigated in the experimental study. The measurements were carried out at three different Reynolds numbers $\left(2025,3290\right.$ and 4555) based on the nozzle diameter $\left(d_{0}=5.8 \mathrm{~mm}\right)$. Further details on the experimental setup are found in Chapter 6.

The experimental results were used to analyze the behavior of the investigated confluent jets and provide information on Reynolds number dependence. The experimental results are also used both when constructing and validating the CFD simulations in the computational study, explained below.

\section{- Computational study}

The computational study makes use of CFD simulations to simulate the same $6 \times 6$ confluent jet array as in the experimental study at Reynolds number 3290. The results from the experimental study were used both to create a proper mesh design and to provide inlet boundary conditions to the CFD simulations. The CFD simulations are performed using three different RANS turbulence models, two two-equation models (standard $k-\varepsilon$ and RNG $k-\varepsilon$ ) and the second-order closure Reynolds Stress Model (RSM). The three used turbulence models were chosen as they represent models of different levels of complexity, computational resource demand and provide different level of description of the turbulent flow field. The turbulence models are referred to below only as $k-\varepsilon s t d$., RNG $k-\varepsilon$ and RSM. The results from the CFD simulations are compared to experimental data in terms of both mean velocity and turbulent properties. The validated CFD results provide data at all locations in the solution domain and the computational results are therefore used to further analyze the investigated confluent jet array.

The basis of CFD, turbulence models that have been employed, and other numerical aspects are presented in Chapter 4. Information on the CFD model in the present work and the use of experimental data as inlet boundary conditions are presented in Chapter 7. 


\section{- Parametrical study}

In addition to the investigation of Reynolds number dependency in the experimental study an extensive parametrical study was carried out to investigate the influence of array size (number of jets), jet spacing, nozzle diameter, inlet velocity and nozzle height. The parametrical investigation makes use of CFD simulations and statistical approximation methods, through Response Surface Methodology and Box-Behnken factorial designs. The statistical methods are described in Chapter 5.

The result provides information on how the investigated parameters influence a number of important aspects related to confluent jet behavior. The resulting Response Surface (RS) models may also be used to provide estimates of the investigated properties for any custom combination of the input parameters within the investigated solution domain. The accuracy of the RS models was evaluated by its capability to reproduce the results of validated CFD simulations in the computational study.

\subsection{Limitations}

The present work consists of computational, experimental and statistical investigations. The experimental investigations are limited to the region from the nozzle exit up to a maximum downstream distance of $29 d_{0}$. The computational investigations extend up to a maximum downstream distance of $150 d 0$. Far-field behavior beyond $150 d_{0}$ is not investigated. All setups, both experimental and computational, are isothermal, and at low Reynolds numbers. Computational results make use of Reynolds averaging and experimental results are reported as ensemble averages. Unsteady, time-dependent phenomena have not been considered. The study is limited to an in-line array of nozzles; other configurations, such as hexagonal or staggered arrays, have not been investigated.

As for the experiments they are limited to PIV and LDA measurements, which both bring a degree of measurement uncertainty. The PIV measurements are limited to measurements of two instantaneous velocity components and the LDA measurements to the stream-wise velocity component only. The size and optical access to the test chamber also brings certain limitations to possible measurement locations. The PIV post-processing is performed using the commercial software $\mathrm{DaVis}$, which brings a limited number of processing options.

The CFD simulations are limited to steady-state RANS modeling using $k-\varepsilon$ std., RNG $k-\varepsilon$ and RSM for turbulence modeling. The CFD simulations are limited to the use of the commercial finite volume code ANSYS Fluent. The use of a commercial code brings a limited number of modeling options both in terms of turbulence modeling and numerical aspects, such as discretization and meshing strategies. Additionally, CFD simulations requires large computational resources, which sets limits on size and mesh density of the computational models.

The statistical approximation methods are limited to Response Surface Methodology and full quadratic Box-Behnken factorial designs. 


\subsection{Appended papers}

A total of six papers are included in the thesis. A list of the appended papers is found at the end of this section. Papers I, II and IV have been peer-reviewed and published in international journals. Paper III has been peer-reviewed and presented at an international conference. Paper $\mathrm{V}$ has been peer-reviewed and is accepted for journal publication. Paper VI has been submitted for journal publication.

It is worth mentioning that the papers do not appear in chronological order. Instead they are ordered in accordance with the research process described in Section 1.4. Papers I and II report on the results from the experimental study. The computational study is focused around Papers III and IV, which contains validation of computational models through comparison with experimental data. Paper IV in particular also uses the computational result for further analysis of confluent jet properties. Papers V and VI are almost completely based on CFD simulations and contain the results from parametrical investigations. The connection between the research questions stated in Section 1.3 and the appended papers are visualized by Table 1.

Table 1 Research questions treated in each appended paper.

\begin{tabular}{ccccccc}
\hline $\begin{array}{c}\text { Research } \\
\text { question }\end{array}$ & I & II & III & IV & V & VI \\
\hline Q1 & - & - & - & - & - & - \\
Q2 & & & - & - & - & - \\
Q3 & - & - & & & - & - \\
\hline
\end{tabular}

\section{Paper I}

Ghahremanian S., Svensson K., Tummers M., Moshfegh B. 2014. Near-field development of a row of round jets at low Reynolds numbers. Experiments in Fluids, $55,1-18$.

\section{Paper II}

Ghahremanian S., Svensson K., Tummers M., Moshfegh B. 2014. Near-field mixing of jets issuing from an array of round nozzles. International Journal of Heat Fluid Flow, 47, 84-100.

\section{Paper III}

Svensson, K., Ghahremanian, S., Moshfegh, B., Tummers, M., 2012. Numerical and experimental investigation of flow behavior in a confluent jet ventilation system for industrial premises. Proceedings of the 10th International Conference on Industrial Ventilation, Paris.

\section{Paper IV}

Svensson K., Rohdin P., Moshfegh B., Tummers M. 2014. Numerical and experimental investigation of the near zone flow field in an array of confluent round jets. International Journal of Heat Fluid Flow, 46, 127-146. 


\section{Paper V}

Svensson K., Rohdin P., Moshfegh B. 2015. A computational parametric study on the development of confluent round jet arrays. Accepted for publication in European Journal of Mechanics / Part B, Fluids.

\section{Paper VI}

Svensson K., Rohdin P., Moshfegh B. 2015. On the influence of array size and jet spacing on jet interactions and confluence in round jet arrays. Submitted for journal publication.

\subsection{Co-author statement}

The experimental studies were planned by $\mathrm{PhD}$ student Shahriar Ghahremanian and by Prof. Bahram Moshfegh. The measurements were carried out in close collaboration between the author (Klas Svensson) and $\mathrm{PhD}$ student Ghahremanian. The author carried out the majority of LDA measurements, while the majority of PIV measurements were done by PhD student Ghahremanian. Both PIV and LDA measurements were performed under supervision of Dr. Ir. Mark Tummers. The measurement results were analyzed, interpreted and put together by the author and PhD student Ghahremanian under the supervision of Prof. Moshfegh and Dr. Tummers. As a result, Paper I and II were written by the author and PhD student Ghahremanian. However, valuable comments and advices were received from Prof. Moshfegh and Dr. Tummers.

The author exclusively conducted all numerical simulations in the thesis, under the supervision of Prof. Moshfegh and Dr. Patrik Rohdin. Papers III-VI have been written entirely by the author. However, valuable comments and advice have been received from the co-authors throughout the whole process from planning the investigations, interpreting the results, and finally improvements on the disposition and contents of the papers. 


\section{Theoretical background and literature review}

This chapter contains a summary of fundamental features previously reported in the literature for single round jets and twin jet configurations. The summary focuses on aspects that are relevant for the present study. The chapter also contains a literature review on multiple round jets.

The present chapter serves to provide a literature review and theoretical background for jet configurations related to the present work. This includes single round jets, twin plane or round jets and multiple round jets.

A single round jet, often referred to as an axisymmetric jet, is considered one of the fundamental free shear flows, and has drawn considerable scientific attention over the years. Investigations on single round jets include aspects such as decay of maximum velocity, spreading rate, regions of development and entrainment of surrounding air.

As the confluent jets in the present study consist of round jets, although interacting, a brief review on the development of a single round jet issuing to a stagnant environment is of interest to the present work.

Among multiple interacting jets much attention has been paid to twin jets, especially plane twin jets, for which three characteristic regions of development have been identified. A review of the implications of jet interactions and the definitions of different regions of development in twin jets are also relevant to the present work.

Finally, scientific contributions also include multiple round jets. While there are differences in geometrical configurations and many of the reported investigations relate to confined jet configurations, a review of relevant literature is still important.

\subsection{Single round jets}

For single round jets three regions of development have been identified. The regions of development are visualized in Figure 3. Next to the inlet is the core region. This region is dominated by the potential core, which has low turbulence and constant velocity.

Depending on the inlet shape, an increase of stream-wise velocity resulting in a saddle-backed velocity profile can occur very close to the inlet. This behavior is 
known as the vena contracta effect and arises due to streamline curvature within the nozzle contraction, appearing in finite-length contractions when the contraction rate is not too small (Morel, 1975). The influence of nozzle shape on the near-zone development of a single round jet has been investigated by Quinn (2006) and Mi et al. (2001).

Initial instability modes created by the jet at its source will produce structures in the jet shear-layer and resulting vortices will experience roll-up and then pair up as the jet evolves (Ball et al., 2012). The turbulent vortices will move downstream within the shear layer, and at the same time grow in size (Todde et al., 2009). The potential core therefore has a conical shape. Eventually the turbulent structures reach to the jet center line, which occurs at the tip of the potential core in Figure 3. At this position the velocity at the geometrical centerline of the jet will start to decrease and the downstream distance to this point is referred to as the potential core length. The length of the potential core is generally a few diameters. As the Reynolds number of the jet increases, the length of the potential core will be reduced (Fellouah et al., 2009; Kwon and Seo, 2005).

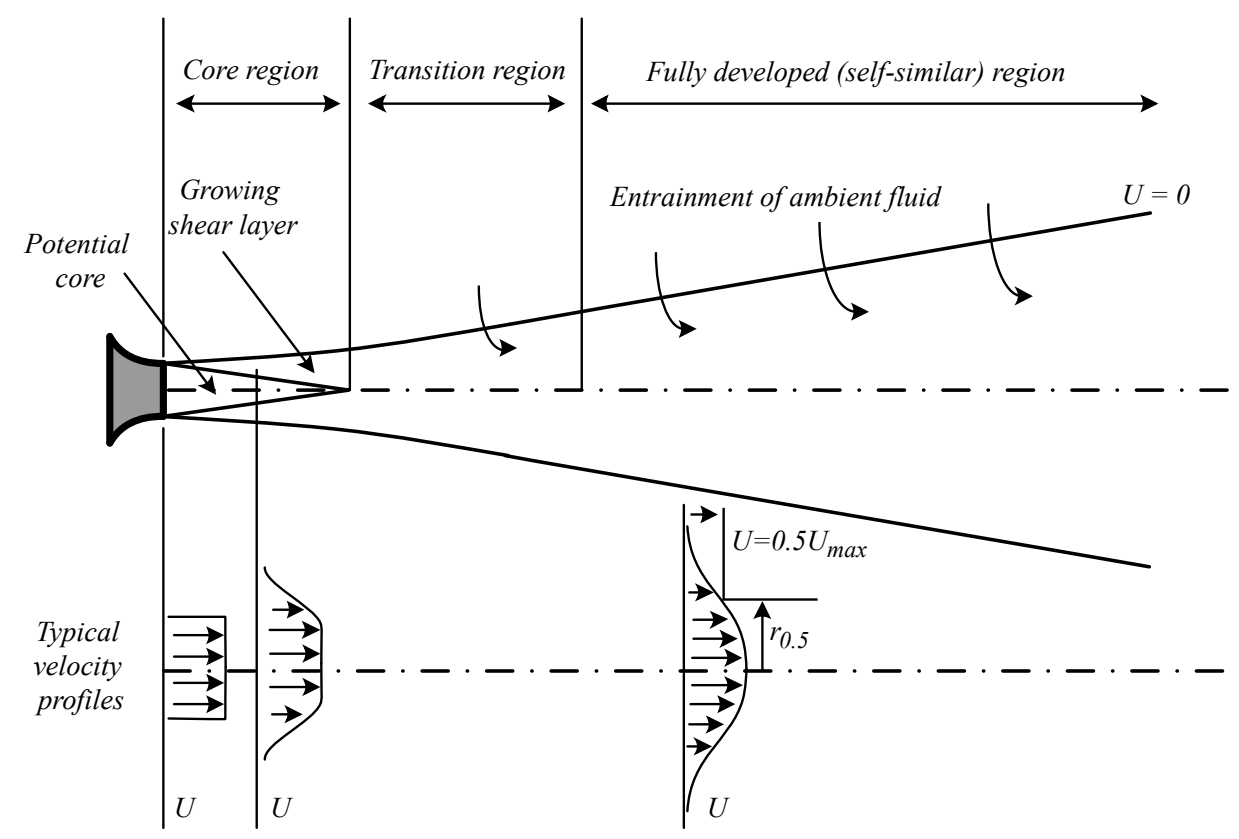

Figure 3 Illustration of an unconfined single round jet.

The second region is called the transition region. In this region the large structures generated in the shear layers surrounding the potential core will break down into smaller structures, of random appearance. Hence the jet will become fully turbulent. A discussion of the formation and breakdown of large-scale vortices close to the inlet is found in Todde et al. (2009). In the transition region the centerline velocity will gradually start to decay.

Eventually the jet become fully turbulent, which is the third region of development, often referred to as the fully developed region. In this region the jet is self-similar and it is this region that is subject to theoretical investigation. As shown by the theoretical reasoning by for example Rajaratnam (1976) the decay of maximum (centerline) velocity $\left(U_{\max }\right)$ is proportional to the inverse of stream-wise distance from the jet inlet 
$(x)$ in this region. In the fully developed region the decay of maximum stream-wise velocity is therefore often described by Eq. (1).

$$
\frac{U_{b}}{U_{\max }}=\frac{1}{K}\left(\frac{x}{d_{0}}-\frac{x_{0}}{d_{0}}\right)
$$

where $K$ is known as the velocity decay coefficient, $U_{b}$ the inlet bulk velocity and $x_{0}$ the virtual origin.

There are numerous experimental investigations which report on the velocity decay coefficient for single round jets $(K)$, where the contributions by Hussein et al. (1994) and Wygnanski and Fiedler (1969) are among the most famous.

The reported values on $K$ commonly span between $4.0-6.7$ (see for example the review articles by Lipari and Stansby (2011) and Abdel-Rahman (2010)), although Malmström et al. (1997) reported values below 4 for jets with very low inlet velocity. For the virtual origin $\left(x_{0}\right)$ reported values in the literature show significant spread. Values between -2.6 and 4.4 are often reported, although Todde et al. (2009) and Wygnanski and Fiedler (1969) reported maximum values of 6.06 and 7 respectively.

Numerical investigations of single round jets have been conducted by Boersma et al. (1998); Ghahremanian and Moshfegh (2014); Olsson and Fuchs (1996); Pope (1978) and Magi et al. (2001).

As the jet interacts with surrounding fluid, not only will the maximum velocity of the jet decrease, the width of the jet will also increase. Usually the half-width $\left(r_{0.5}\right)$, which is computed as the radial distance where the stream-wise velocity is half of its maximum, i.e., $U=0.5 \cdot U_{\max }$, is used as measurement of the jet width. It can be shown theoretically that in the fully developed region a single jet will spread linearly according to Eq. (2).

$\frac{r_{0.5}}{d_{0}}=b\left(\frac{x}{d_{0}}-\frac{x_{0, b}}{d_{0}}\right)$

where $b$ is the spreading rate and $x_{0, b}$ is the virtual origin.

The spreading rate of a single round jet is in the order of 0.1 (see for example Malmström et al. (1997)).

The turbulent structures that the jet develops will entrain fluid from the stagnant surrounding. The volumetric flow rate of an axisymmetric round jet, made dimensionless with the inlet flow rate $\left(Q_{0}\right)$, is computed according to Eq. (3).

$\frac{Q}{Q_{0}}=\frac{2 \pi}{Q_{0}} \cdot \int_{0}^{R} U r d r$

where $R$ is radial location of the jet boundary. 
In theory the stream-wise velocity profile shall be integrating to $R \rightarrow \infty$, in order to consider the whole jet. In practice, some finite criteria of the jet boundary must be employed.

Dimensional analysis shows that in the fully developed region the mass flow rate of the jet increases proportional to the downstream distance (see Rajaratnam (1976)). This means that the entrainment rate is constant when the jet is fully developed and that the flow rate can be computed according to Eq. (4).

$\frac{Q}{Q_{0}}=C_{Q} \frac{x}{d_{0}}$

where $C_{Q}$ is the entrainment coefficient.

The value of $C_{Q}$ needs to be determined experimentally (Rajaratnam, 1976). Ricou and Spalding (1961) reported a value of $C_{Q}=0.32$ for a single round jet, and this observation was supported by Hill (1972) and Han and Mungal (2001). Crow and Champagne (1971) reported a somewhat lower value of $C_{Q}=0.292$.

The above values are for the fully developed region. In the region $x / d_{0}<2$ Crow and Champagne (1971) reported a value of $C_{Q}=0.136$ and Hill (1972) and Han and Mungal (2001) also reported lower values of the entrainment rate close to the nozzles.

\subsection{Twin jets}

Twin jets occur when two parallel jets are issued close to each other. As the jets move downstream they will interact with each other and eventually form a single jet. Most attention has been paid to twin plane jets, as the twin plane jet is favorable for experimental investigation, due to its two-dimensionality.

\subsubsection{Twin plane jets}

Twin plane jets consist of two parallel slot inlets with the same width (w), separated by a certain center-to-center distance $(S)$ (see Figure 4). The development of twin plane jets are often divided into three main regions: converging, merging and combined region. The general developments of twin plane jets are illustrated in Figure 4.

Close to the nozzles mutual entrainment leads to a low (sub-atmospheric) pressure in the space between the jets. The low pressure causes the twin jets to deflect towards each other and between the jets a Counter-Rotating Vortices Pair (CVP) is created (Miller and Comings, 1960; Tanaka, 1970). The CVP causes recirculation and considerable flow reversal on the symmetry plane between the jets. Therefore the area between the jets close to the nozzles is known as the recirculation region (Ko and Lau, 1989) or recirculation zone (Nasr and Lai, 1997).

As the jets on each side of the recirculation zone bend towards the bisecting plane of symmetry, the first region of development is called the converging region. The converging region extends from the inlet up to the location of the free stagnation 
point that occurs at the symmetry plane, at the end of the recirculation zone. The converging region includes both the potential core of the two jets and the recirculation zone (see Figure 4).

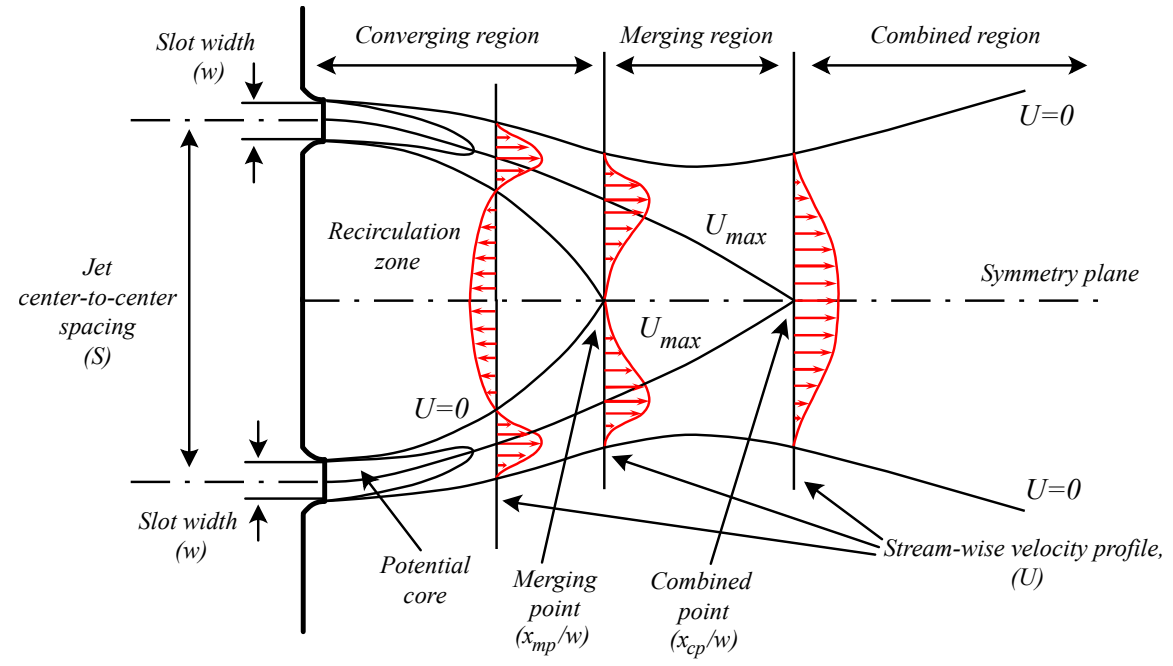

Figure 4 Illustration of twin plane jets (based on Nasr and Lai (1997)).

As the jets curve towards the symmetry plane, due to the asymmetrical mixing, the outer edge of the jet experiences larger stream-wise turbulence than the inner edge due to centrifugal forces and deflection of the jets (Nasr and Lai, 1997; Tanaka, 1970). The free stagnation point at the symmetry plane is referred to as the merging point and is considered the beginning of the merging region. In the merging region the interactions between the two jets increase and the velocity at the symmetry plane increases until it reaches a maximum.

The location where the velocity at the symmetry plane reaches its maximum is called the combined point and denotes the end of the merging region and the beginning of the combined region. For twin plane jets this coincides with the stream-wise location at which the maximum stream-wise velocity occurs on the symmetry plane (opposed to two peaks) (Anderson and Spall, 2001). Some authors also used an extrapolation of the jet central streamline to the symmetry plane as combined point location (Fujisawa et al., 2004; Murai et al., 1976). In the combined region the jets form a combined jet which resembles a single jet.

The locations of merging $\left(x_{m p} / w\right)$ and combined points $\left(x_{c p} / w\right)$ are of considerable interest when studying twin plane jets. The dimensionless jet spacing, defined as $S / w$ (see Figure 4), is an important parameter for twin jet flows and the effects of jet spacing on velocity decay, jet spreading, momentum and volumetric flow rates, etc. has been studied by Nasr and Lai (2000). There are also several experimental investigations reporting the locations of merging and combined point, at different jet spacing.

CFD simulations of twin plane jets has been conducted by Durve et al. (2012) who used the results together with experimental data in the literature to predict the location of merging and combined points based on nozzle spacing and inlet turbulence intensity $\left(T_{U}\right)$. Durve et al. (2012) also concluded that the CFD simulations 
compared well with the experimental data. Numerical simulations on twin plane jets have also been conducted by Anderson and Spall (2001) who reported that both the $k-\varepsilon s t$. and RSM were able to accurately predict the location of merging and combined points. Three different turbulence models: the $k-\varepsilon s t d$., RNG $k-\varepsilon$, and RSM model were evaluated by Lai and Nasr (1998). They found that the main features of twin plane jets were captured by all models.

In Table 2 reported values and empirical correlations for the two characteristic points, found in the literature, are summarized. The review of data is limited to twin plane jets with closely spaced jets $(S / w \leq 8)$, since only these are relevant to the present work. A summary of reported values for twin plane jets with larger jet spacing can be found in Nasr and Lai (1997).

Table 2 Location of merging and combined points of twin plane jets reported in the literature.

\begin{tabular}{|c|c|c|c|c|}
\hline Author & $\begin{array}{l}\text { Spacing } \\
S / w\end{array}$ & $\begin{array}{c}\text { Reynolds } \\
\text { number } \\
R e_{w}\end{array}$ & $\begin{array}{c}\text { Merging point } \\
\text { location } \\
x_{m p} / w\end{array}$ & $\begin{array}{c}\text { Combined point } \\
\text { location } \\
x_{c p} / w\end{array}$ \\
\hline $\begin{array}{l}\text { Miller and Comings } \\
\qquad(1960)\end{array}$ & 6 & 17,800 & 6.8 & 12.4 \\
\hline Murai et al. (1976) & 4.1 & $3,350-18,600$ & - & 9.8 \\
\hline Ko and Lau (1989) & 2.5 & 2,900 & 1.75 & 10.5 \\
\hline Nasr and Lai (1997) & 4.25 & 11,000 & 4 & 8 \\
\hline Nasr and Lai (2000) & & & $1.049(S / w)^{0.948}$ & $3.514(S / w)^{0.673}$ \\
\hline $\begin{array}{l}\text { Fujisawa, Nakamura et al. } \\
\text { (2004) }\end{array}$ & 3 & 1,200 & 3 & 6 \\
\hline Durve et al. (2012) (CFD) & 3.5 & 6,000 & 2.7 & 7.1 \\
\hline Durve et al. (2012) & & & $\begin{array}{c}0.721 S / w+ \\
2.06 T_{u}-2.453\end{array}$ & $\begin{array}{c}1.231 \mathrm{~S} / w+ \\
2.06 T_{u}-2.453\end{array}$ \\
\hline
\end{tabular}

\subsubsection{Twin round jets}

Twin round jets are similar to twin plane jets, however, the two slots having a width of $w$ are replaced with two round inlets with diameter, $d_{0}$. In a twin round jet setup the recirculation zone of trapped fluid between the jets, which is characteristic for twin plane jets, will be much weaker or not occur at all (Baratian-Ghorghi et al., 2012; Vouros and Panidis, 2008). This requires another definition of the merging point in twin round jets, compared to twin plane jets, as the free stagnation point at the bisecting symmetry plane might not exist. Attempts to define a criterion for the merging point in twin round jets has been made by Vouros and Panidis (2008), who suggested defining the merging point as the location where the minimum stream-wise velocity between the jets exceeds $10 \%$ of the maximum stream-wise velocity in the flow field, i.e., $0.1 \cdot \max (U)$

The experimental investigation by Okamoto et al. (1985) showed that, similar to twin plane jets, twin round jets interact and join to a single jet as the position of maximum velocity shifts from the nozzle axis to the symmetry plane between the jets. An important difference between twin round jets and twin plane jets is that for twin 
round jets the maximum velocity at the symmetry plane occurs upstream of the position where the twin round jets share a common maximum in stream-wise velocity, opposed to two off-centered peaks on each side of the symmetry plane. This behavior was clearly shown in the experimental investigation by Harima (2001).

In Table 3 the location of merging point, maximum velocity at the symmetry plane and combined point (a shared maximum of stream-wise velocity at the symmetry plane) reported in the investigation by Harima (2001) are shown. Twin round jets have also been investigated by Meslem et al. (2010) and reported values of the combined point location is added in Table 3.

Table 3 Location of merging and combined points reported for twin round jets.

Merging point location is extracted as the location where the velocity at the symmetry plane is equal to $0.1 \cdot U_{b} \approx 0.1 \cdot \max (U)$.

\begin{tabular}{cccccc}
\hline Author & $\begin{array}{c}\text { Spacing } \\
S / d_{0}\end{array}$ & $\begin{array}{c}\text { Reynolds } \\
\text { number } \\
R e_{d}\end{array}$ & $\begin{array}{c}\text { Merging point } \\
\text { location } \\
x_{m p} / d_{0}\end{array}$ & $\begin{array}{c}\text { Maximum } \\
\text { velocity at } \\
\text { symmetry plane }\end{array}$ & $\begin{array}{c}\text { Combined } \\
\text { point location } \\
x_{c p} / d_{0}\end{array}$ \\
\hline Harima (2001) & 2 & & 2 & 12 & 25 \\
\hline $\begin{array}{c}\text { Meslem et al. } \\
(2010)\end{array}$ & 25 & 25,000 & 9 & 22 & 52 \\
\hline
\end{tabular}

The combined point is located further downstream in twin round jets compared to twin plane jets. Even the location of maximum velocity at the symmetry plane, which occurs substantially upstream of the combined point in twin round jets, is located at larger downstream distance compared to the combined point of twin plane jets. There is rather good agreement in terms of combined point location in the two investigations on twin round jets, although the value reported by Meslem et al. (2010) is somewhat lower.

For the merging point location there is an important difference in the results by Harima (2001) and Meslem et al. (2010). In the study by Meslem et al. (2010) the velocity at the plane of symmetry is always higher than $0.1 \cdot \max (U)$ for $x / d_{0} \geq 2$. This means that merging is present already at $x / d_{0}=2$, which is the most upstream reported measurement location.

In the study by Harima (2001) the merging point of twin round jets occurs further downstream in comparison with twin plane jets, i.e., the mixing layer interactions will start further downstream in twin round jets with the same dimensionless jet spacing $\left(S / d_{0}\right.$ or $\left.S / w\right)$. It shall be noted that the definition of merging point slightly differs between the two configurations, as the merging point location is computed as $U=0$ in twin plane jets, and as $U=0.1 \cdot \max (U)$ for twin round jets. However, as shown by the results in Nasr and Lai (1997) and Nasr and Lai (1997), the velocity between twin plane jets will increase rapidly closely downstream of the merging point. Therefore, the slightly different definition of the merging point for the twin jets will not change the observation described above. 
Harima (2001) concluded that downstream of the combined point the maximum stream-wise velocity of twin round jets was higher compared to a single round jet, and decreasing nozzle spacing leads to higher jet velocity. In the merging region Meslem et al. (2010) reported lower values of maximum velocity for the twin round jets compared to a single jet. Harima (2001) concluded that the entrainment close to the nozzles $(S / w<20)$ is lower for twin round jets compared to a single round jet and decreases when $S / w$ decreases. In the downstream region $(S / w \geq 20)$ the entrainment rate was similar between single round jets and twin round jets. A similar trend was reported by Meslem et al. (2010) where the entrainment in twin round jets was lower compared to a single jet upstream of $x / d_{0}=4$. Downstream of this location the entrainment increased and attained larger values compared to a single round jet.

Further investigation on development of turbulence intensity and Reynolds stresses in twin round jets has been conducted by Harima et al. (2005), which revealed a switching tendency between major and minor axis of stream-wise turbulence intensity contours for twin round jets with small jet spacing. It was also shown that the development of turbulence intensity at each circular jet center is suppressed by the interference between the jets (Harima et al., 2005). Allen and Smith (2011) performed CFD simulations using the $k-\varepsilon$ std. model, comparing their results with the experimental data by Harima et al. (2005). The results showed that the $k-\varepsilon$ std. model could accurately predict the development of turbulence intensity.

Yin et al. (2007) investigated the influence of Reynolds number on development of twin round jets and found that increasing Reynolds numbers leads to stronger jet interactions and higher level of turbulent kinetic energy.

\subsection{Multiple round jets}

Multiple jets in various configurations, both confined and unconfined multiple jets, have previously been subject to scientific investigations. Early contributions on a single row of jets, consisting of either slot, round or rectangular jets, have been reported by Corrsin (1944); Knystautas (1962); Pani and Dash (1983) and Marsters (1979). These investigations resulted in two main findings: the combining of jets into a single jet and the deflection of side jets towards the center of the row.

Knystautas (1962) studied a row of practically infinite number of circular jets. In Table 4 the locations of maximum velocity at the symmetry plane between jets and the combined point are presented. A comparison with the reported values for twin round jets (see Table 3) shows that the locations of these characteristic points are of similar magnitude. 
Table 4 Location of combined points reported by Knystautas (1962) for infinite row of round jets.

\begin{tabular}{cccccc}
\hline Author & $\begin{array}{c}\text { Spacing } \\
S / d_{0}\end{array}$ & $\begin{array}{c}\text { Reynolds } \\
\text { number } \\
R e_{d}\end{array}$ & $\begin{array}{c}\text { Merging point } \\
\text { location } \\
x_{m p} / d_{0}\end{array}$ & $\begin{array}{c}\text { Maximum } \\
\text { velocity at } \\
\text { symmetry plane }\end{array}$ & $\begin{array}{c}\text { Combined } \\
\text { point location } \\
x_{c p} / d_{0}\end{array}$ \\
\hline $\begin{array}{c}\text { Knystautas } \\
(1962)\end{array}$ & 1.5 & $292,000-$ & - & 7 & 20 \\
& 2 & $2,530,000$ & - & 11 & 28 \\
\hline
\end{tabular}

Knystautas (1962) also concluded that a combined jet behaves as a two-dimensional slot jet, which means that maximum stream-wise velocity will be inversely proportional to the square root of the downstream distance $\left(U_{\max } \propto 1 / \sqrt{x}\right)$ instead of $U_{\max } \propto 1 / x$, which is the relation for single round jets. Pani and Dash (1983) studied a limited number of round jets in a row and showed how the maximum velocity in the center of the row gradually started to decay according to $1 / \sqrt{x}$ as the number jets increased.

As reported in the two previous sections, jet convergence is an important aspect of twin jet flow. Jet convergences have also been shown in a multiple round jet configuration by Yimer et al. (1996) who studied a multi-jet burner with a ring of either four or six jets surrounding a central port. The result showed that the outer jets curve towards the center jet, while merging with each other and the jet in the center. Yimer et al. (1996) concluded that this overall contraction is a result of the entrainment action of each jet, which draws in fluid from its surroundings and therefore produces suction on its neighbors.

A detailed analysis of multiple jets emerging through a perforated plate was conducted by Villermaux and Hopfinger (1994). Contrary to the investigation by Yimer et al. (1996) there was no entrainment of ambient air from the sides in this setup as the perforated plate was extended all the way to the walls of the wind tunnel. Therefore the merging of the multiple jets, still present in this configuration, is due to jet spreading rather than the deflection of jets towards the center. The results showed that the merging length of the jets was both oscillating and Reynolds numberdependent below a Reynolds number of 3,000 - 4,000. An unstable behavior was also reported for multiple plane jets by Corrsin (1944).

Further analysis on multiple jets has been conducted within research related to combustion or chemical mixing in Tatsumi et al. (2010) and Boutazakhti et al. (2007) and for ventilation supply devices by Meslem et al. (2010). The study by Meslem et al. (2010) showed that for an array of round jets the entrainment rate was higher close to the inlet and reducing in the downstream direction, contrary to twin round jets.

Closely spaced jets in an opposed jet burner issuing through turbulence-generating plates have been investigated experimentally by Böhm et al. (2010) and computationally using LES by Stein et al. (2011) and using both LES and DNS by Rieth et al. (2014). In both CFD simulations and experiments three regions were found for the jets in the turbulent opposed burners. Close to the nozzle plate is an 
initial region with constant jet velocity and low velocity between jets. The region that follows is dominated by strong jet-to-jet interactions and decay in jet velocity. Then, at the final stage, turbulence decays and individual jets can no longer be seen (Böhm et al., 2010; Stein et al., 2011).

Multiple round jets impinging on a flat plate have been studied experimentally by Geers et al. (2006) and Geers et al. (2005) and numerically by Thielen et al. (2005) and Thielen et al. (2003). The results show both strong jet-to-jet interactions and symmetry-breaking phenomena within multiple impinging jet configurations. 


\section{Measurement techniques}

This chapter provides information on the measurement techniques, Laser Doppler Anemometry (LDA) and Particle Image V elocimetry (PIV), used in the experimental part of the present work. Working principles, main components of measurement equipment, calibration procedure and postprocessing options are described. In the last section of the chapter a short comparison of the two techniques is provided.

Techniques for measuring mean velocity and turbulent fluctuations may be classified as intrusive or non-intrusive and as point or whole-field measurement techniques. With intrusive measurement techniques equipment needs to be placed at the measurement location. This causes disturbances to the flow, which in some cases might affect the measurement results. With non-intrusive measurement techniques no measuring equipment needs to be placed at the measuring location. Measurements of velocity in air flows commonly rely on one of the three following principles: pressure measurement, thermal anemometry or tracer particles.

Measurements based on pressure or thermal (hot-wire) anemometry are both intrusive and point measurement techniques. Measuring techniques related to pressure rely on measurement of both static and total pressure, using pressure probes (Pitot tubes), in conjunction with the Bernoulli equation. This measurement technique is perhaps the most commonly used means of determining fluid velocity in a steady, mid-to-high velocity flow (Foss et al., 2007).

Thermal anemometers measure fluid velocity by sensing the changes in heat transfer from a small, electrically heated sensor exposed to the fluid motion (Goldstein, 1996). Measurements with thermal anemometers have been employed in a widespread number of flow problems. The main advantage with thermal anemometers is that measurements can be made with high temporal resolution that enable spectral analysis of turbulence. However, stationary thermal anemometers cannot distinguish flow reversal and thermal anemometers require calibration towards known velocities.

When tracer particles are used, non-intrusive measurements can be performed with for example Laser Doppler Anemometry (LDA), Laser Transit Velocimetry (LTV) and Laser Phase Doppler (LPD). All of the above are point measurement techniques. Particle Image Velocimetry (PIV), Particle Streak Velocimetry (PSV) or Doppler Global Velocimetry (DGV) are examples of non-intrusive whole-field measurement techniques. Except for PSV all the above methods can detect flow reversals. 
The present work uses non-intrusive measurement techniques, either as point measurement with LDA or whole-field field measurements with planar PIV. The use of non-intrusive measurement techniques is beneficial to the present investigations as the geometrical size of the experimental setup is relatively small (see Chapter 6), making it difficult to conduct measurements in the central part of the jet array without sensors or mountings disturbing the surrounding environment. The two employed measurement techniques are presented in the following sections.

\subsection{Laser Doppler Anemometry (LDA)}

In a dual beam LDA system the velocity of individual particles travelling with the flow are determined by measuring the Doppler-shift frequency of the scattered light as the particle passes through two intersecting beams of coherent light with known wavelength. LDA can be applied to transparent or semi-transparent flows and is known for its high accuracy. A more extensive description of LDA principles may be found in Absil (1995); Albrecht (2003) and Boutier (2013).

\subsubsection{Working principles}

The working principle of a dual beam LDA system may be explained with the fringe model. The fringe model states that when two beams of coherent light, with the same wavelength, are crossed an interference pattern is generated in the probe volume, created in the intersection region of the two beams. A sketch of the wave front propagation and interference pattern in the probe volume is illustrated in Figure 5.

The distance between two intensity maxima in the probe volume interference pattern $\left(d_{f}\right)$ can be determined by Eq. (5).

$d_{f}=\frac{\lambda}{2 \sin (\theta / 2)}$

where $\lambda$ is the wavelength of the (laser) light and $\theta$ is the angle between the intersecting laser beams.

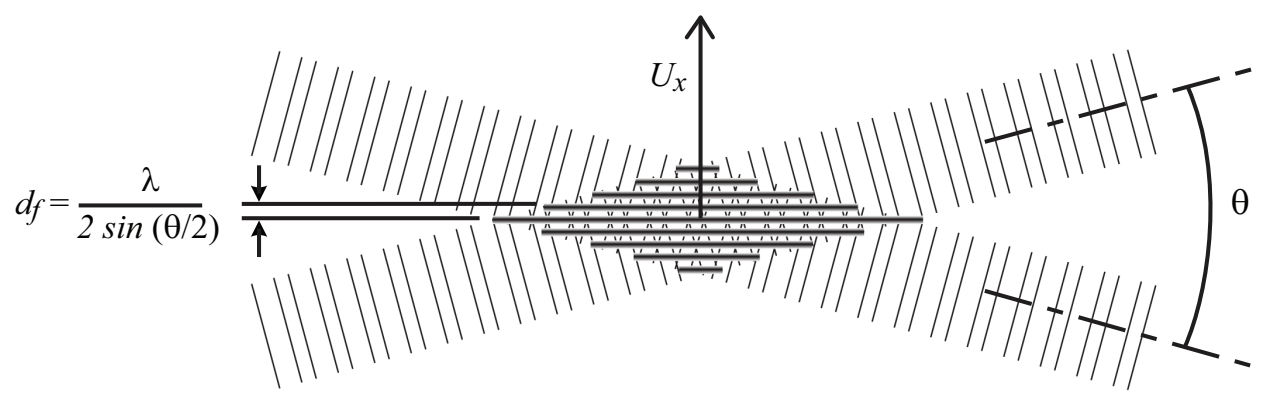

Figure 5 Fringe model, two intersecting laser beams and resulting interference pattern.

When a tracer particle passes the probe volume it will scatter the laser light. The scattered light is recorded on a photo detector, which results in a typical Doppler burst signal shown in Figure 6. After filtering the Doppler burst signal, removing the pedestal, a clean Doppler signal is held. The measured Doppler frequency, $f_{D}$, 
represents the inverse of the time it takes for the tracer particle to travel between two intensity maxima in the fringe pattern. Consequently, the velocity component normal to the fringe pattern $\left(U_{x}\right)$ may be computed according to Eq. (6).

$U_{x}=d_{f} \cdot f_{D}$

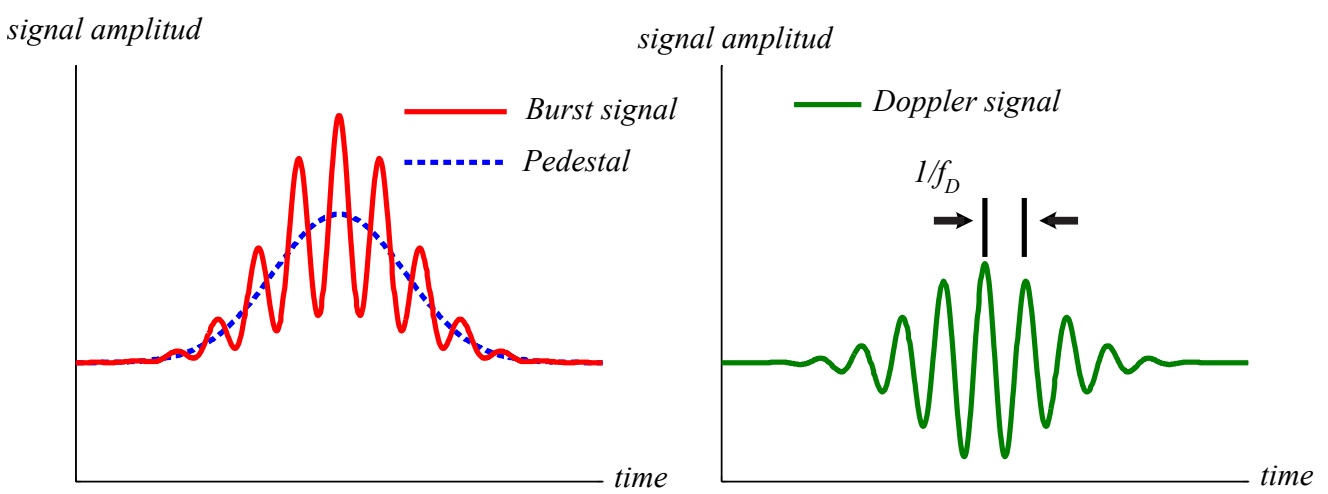

Figure 6 Typical burst signal with pedestal (left), filtered Doppler signal (right).

\subsubsection{Measurement equipment}

The main components of a dual beam LDA system are illustrated in Figure 7. The light source consists of a laser which emits a continuous beam of laser light. A beam splitter spits the beam in two beams of equal light intensity. After the beam splitter, one of the laser beams is frequency shifted in a Bragg cell. A Bragg cell is an opticacoustic cell in which the laser beam is passed through a transparent medium, i.e., a glass block, to which a crystal controlled oscillator has been employed to generate a traveling acoustic wave (Absil, 1995). The Doppler effect will shift the frequency of the laser light passing the Bragg cell. A typical value of the frequency pre-shift $\left(f_{\text {shift }}\right)$ is $40 \mathrm{MHz}$, which was also used in the present work.

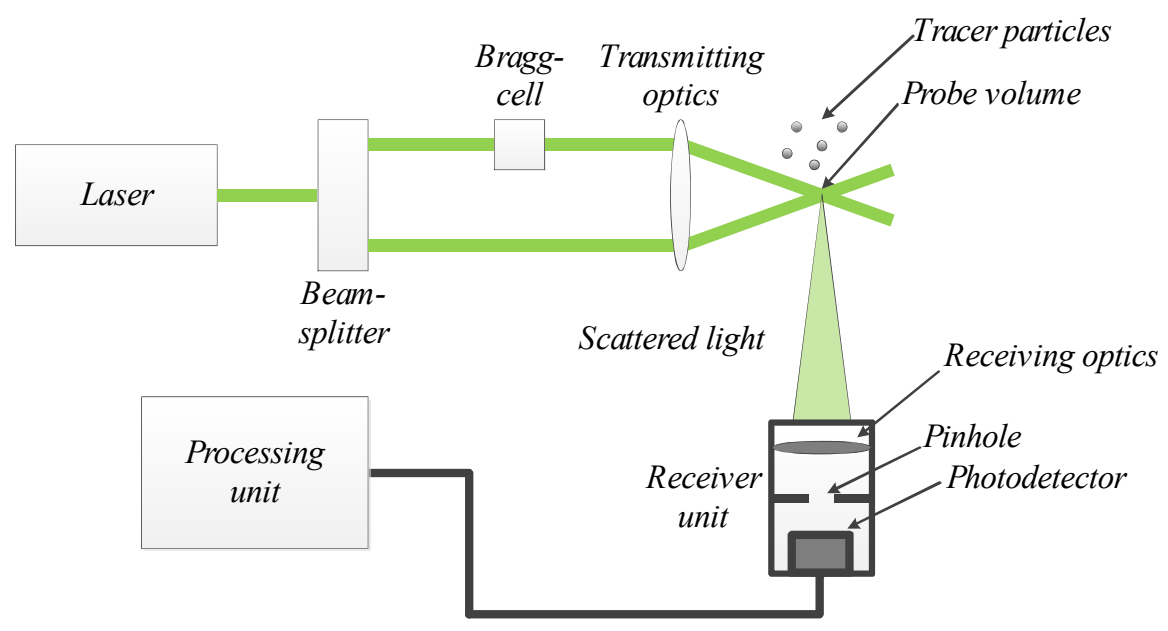

Figure 7 Components in an LDA system.

When one of the beams is pre-shifted this results in a shift in the linear relationship between the measured Doppler frequency, $f_{D}$, and the velocity, $U_{x}$. In the fringe model this represents a moving fringe pattern, with velocity $d_{f} \cdot f_{\text {shift }}$. Particles 
moving against the fringe patterns result in measured Doppler frequency higher than the pre-shift frequency $\left(f_{\text {shift }}\right)$. For particles moving in the same direction as the fringe pattern the measured Doppler signal will be lower than $f_{\text {shift }}$. Thus, the frequency pre-shift is used to determine the direction of the tracer particles, which otherwise would not be possible. The velocity of a particle passing the probe volume is then calculated according to Eq. (7).

$U_{x}=d_{f} \cdot\left(f_{D}-f_{\text {shift }}\right)$

After the Bragg cell the laser beams are transferred, often using fiber cables, to the laser head(s) where the transmitting optics focus the laser beams towards the probe volume. It is important that the beam waists, i.e., focus point of the laser beams, coincide with the probe volume. If the laser beams are not properly focused to the probe volume, wave front curvature will cause variations of spatial frequency, which reduces measurement accuracy (Albrecht, 2003).

Detection of scattered light is achieved using photodetectors. The photodetector converts the amount of collected light to a voltage signal. The receiver unit is also equipped with optics focusing the scattered light to the detector. Often a pinhole is placed in front of the photodetector to reduce the effective size of the measurement volume, by blocking light from the outer part of the probe volume. In the present work light was collected at approximately $90^{\circ}$ off axis, i.e., side scatter. A pinhole was then used to reduce the effective measurement volume length from approximately 1 $\mathrm{mm}$ to $0.15 \mathrm{~mm}$.

With usual Bragg cells the signal processor would need to process signals with frequencies higher than $40 \mathrm{MHz}$, which is usually processed with reduced accuracy compared to lower frequencies (Boutier, 2013). To circumvent this problem the output signal is electronically down-mixed before the photodetector signal is processed.

Although the present work only includes 1D LDA measurements, it is worth mentioning that two- and three-component LDA measurements may be accomplished to crossing two or three pairs of laser beams with different light wavelength (colors) at the same location and collecting light of different colors on separate photodetectors.

\subsubsection{Tracer particles}

The use of LDA requires particles of sufficient resolution present in the flow. The particles should both follow the flow movement accurately and scatter sufficient laser light, to yield a high signal-to-noise ratio of the recorded Doppler signal.

In order to track fluid movement in a precise manner, tracer particles must have properties that are close to those of the fluid, and should be as small as possible (Boutier, 2013). Using Stokes law the tracer particle behavior under acceleration may be derived according to Eq. (8). 
$U_{s}=d_{p}^{2} \frac{\left(\rho_{p}-\rho\right)}{18 \mu} a$

where $U_{s}$ is the velocity lag of a particle, $d_{p}$ is the particle diameter, $\mu$ the dynamic viscosity of the fluid, $\rho$ and and $\rho_{p}$ the density of the fluid and tracer particles and $a$ the fluid acceleration (Raffel et al., 2007).

A primary source of error is the influence of gravitational forces if the densities of the fluid $\rho$ and the tracer particles $\rho_{p}$ do not match (Raffel et al., 2007). Additionally it is also seen that if the particle diameter is increased the velocity lag will also increase.

As mentioned above, the particles must not only follow the fluid movements. They should also scatter light with sufficient intensity. The fact that light scattering improves with increasing particle size, calls for the need of a compromise regarding tracer particle size (Albrecht, 2003).

For experiments in air flows where natural seeding is not sufficient, oil droplets generated with a Laskin nozzle seeding generator may be used as tracer particles. In a Laskin nozzle seeding generator pressurized air is injected to liquid oil in a closed container through a number of small nozzles. This will create aerosol particles that are carried within air bubbles towards the liquid oil surface. An impactor plate with a small opening along the container sides prevents large particles from escaping the container while small oil droplets are able to leave the container through the exhaust. Oil droplets generated by Laskin nozzle seeding generators using vegetable oils, as were used in the present work, generally lead particles with mean diameter of about 1 $\mu m$ (Raffel et al., 2007). A schematic illustration of a Laskin nozzle seeding generator is found in Figure 8.

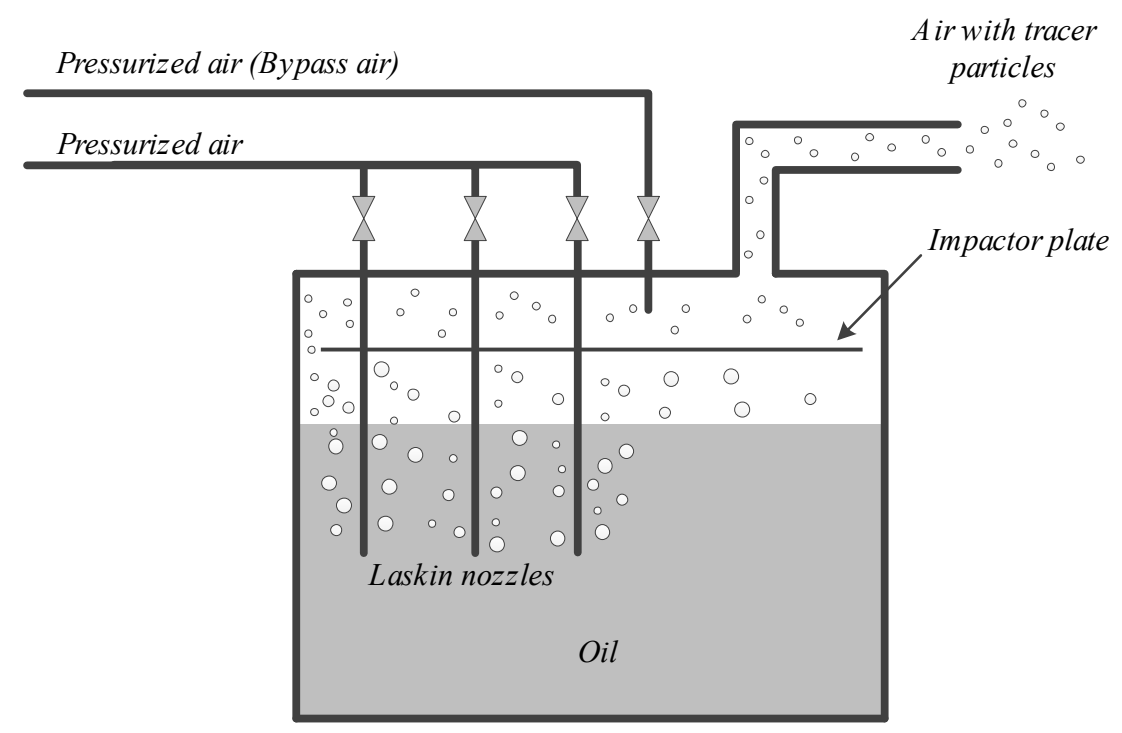

Figure 8 Schematic of Laskin nozzle seeding generator.

The light scattering of small particles depends on the ratio of the refractive index between particle and surrounding fluid, particle size, shape and orientation, but also on polarization and observation angle (Raffel et al., 2007). The highest light intensity 
is found in forward scatter, which is the most beneficial positioning of the LDA receiving unit in terms of light scattering. Backward scatter also has relatively high light intensity and is often used in LDA systems as it simplifies simultaneous traversing of transmitting and receiving units. Backward scatter also enables compact LDA probes containing both transmitting and receiving units. When collecting light at offset axis angles that approach $90^{\circ}$, i.e., side scatter, the effective probe volume length may be minimized, but with the major drawback that the scattered light intensity becomes lower (Boutier, 2013).

\subsubsection{Post-processing and velocity bias}

As an LDA system measures the velocity of individual tracer particles, the output from an LDA measurement will not consist of a continuous signal, with constant sampling frequency. Instead the sampling frequency of LDA data is irregular and depends on the arrival of tracer particles in the measurement volume. Additionally, LDA data is not randomly sampled over time, as generally there is also a correlation between short-term rate of random particle arrivals and the measured flow quantity (Albrecht, 2003). In other words, there is a higher probability to detect particles with high velocity compared to particles with lower velocity, as the rate at which particles pass the measurement volume increases when the velocity is higher. For this reason computing velocity statistics, such as mean velocity and variance, using arithmetic mean may lead to incorrect results. There are two principal different methods for removing velocity bias in LDA data: sampling techniques and weighting factors.

Sampling techniques attempt either to reconstruct the original velocity signal or sample the velocity at approximately equidistant times, thereby eliminating the correlation between the sampling process and the instantaneous velocity (Tummers, 1999). Velocity statistics from the processed signal are then calculated using arithmetic mean. However, the use of sampling techniques requires high sampling rates.

Instead of sampling techniques, weight factors of the velocity samples can be used. A correct weight factor must weight each sample with a factor, $\omega_{p}$, that is inversely proportional to the conditional probability density of a particle arrival at time, $t_{p}$, given the particle velocity $u_{p}$ (Albrecht, 2003). Eq. (9) shows how a weight factor $\omega_{p}$ is used to estimate mean velocity $U$ from velocity measurements of tracer particles with velocity $u_{p}$.

$U=\frac{\sum_{p=1}^{N} u_{p} \omega_{p}}{\sum_{p=1}^{N} \omega_{p}}$

The weighting factor may be based on the inverse of particle velocity, transit time, i.e., the time the particle is located in the measurement volume or the interarrival time, which is the time between two consecutive samples. For the 1D LDA measurements in the present study the use of inverse velocity as weighting factor is not suitable as it requires $3 \mathrm{D}$ LDA measurements to determine the velocity magnitude. According to Albrecht (2003), inverse velocity as weighting factor, based on a single velocity component, is not recommended. Interarrival time weight factors may only be used at 
high data rate. Transit time weighting factors do not require measurements of all velocity components and can also be employed at low data rates. Transit time weighting is the recommended method for situations with spatially homogeneous particle seeding (Albrecht, 2003). For the above reasons transit time weighting according to Eq. (10) is used in the present study.

$\omega_{p}=t_{p}$

where $t_{p}$ is the time the tracer particle remains in the measurement volume.

\subsection{Particle Image Velocimetry (PIV)}

The basic idea in PIV is to illuminate the tracer particles twice and at the same time acquire two images of the particles separated by a certain time interval, $\Delta t$. The displacement of the particles between the images can then be used to evaluate the instantaneous velocity.

PIV is a whole-field measurement technique where the two in-plane instantaneous velocity components are acquired at a large number of locations located on a thin laser sheet plane. The PIV technique may be extended to Stereo PIV, including the out-of-plane velocity component, by using two cameras or even full volumetric PIV (tomography) using at least four cameras. Single-exposure, double-frame, planar PIV, where two consecutive images are recorded on two separate frames using a single camera, has been used in the present work and is explained below.

\subsubsection{Working principles}

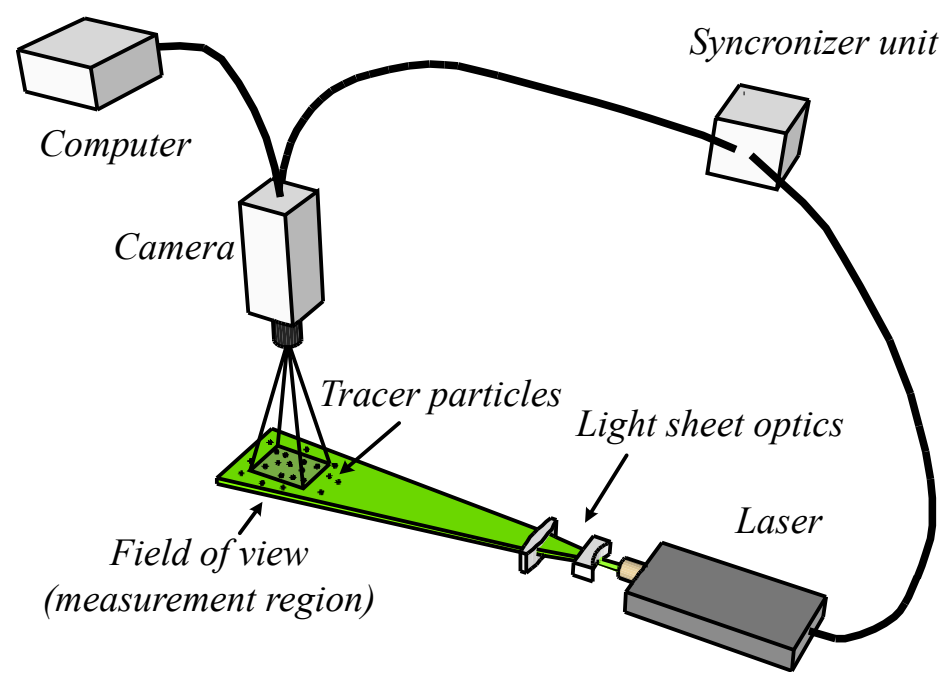

Figure 9 Schematic drawing of PIV setup.

A typical PIV setup is illustrated in Figure 9. A dual-cavity laser is used to generate two laser light pulses, with a certain time delay $(\triangle t)$. Using a combination of lenses, often referred to as light sheet optics, the output laser beam is transformed to a laser light sheet, with a typical thickness of $1 \mathrm{~mm}$ or less. A digital camera is mounted perpendicular to the laser sheet. A synchronizing unit is used to trigger both laser pulses and image acquisition of the camera, thereby capturing images of the 
illuminated particles. The resulting images are transferred to a computer for postprocessing. Using cross-correlation techniques, the particle displacement between the first and second image is computed in a large set of small regions of the images, called interrogation areas. The two in-plane instantaneous velocity components are then derived from the known displacement and time delay between exposures according to:

$u, v=\frac{(\triangle \tilde{x}, \triangle \tilde{y})}{\triangle t}=\frac{(\triangle X, \triangle Y)}{M_{0} \triangle t}$

where $(\triangle \tilde{x}, \triangle \tilde{y})$ is the (physical) particle displacement, $(\triangle X, \triangle Y)$ the particle displacement on the image plane, and $M_{0}$ the magnification factor.

\subsubsection{Lasers and light sheet optics}

In general, when performing PIV measurement, the duration of each illumination (laser pulse) is short relative to the delay between exposures. Therefore it is more efficient to use pulsed lasers compared to a continuous laser. A common laser type used in PIV systems is the frequency doubled Nd:YAG laser. This laser type is used in the present work and is explained below.

An Nd:YAG laser can deliver a pulse energy of $10-10^{3} \mathrm{~mJ}$ and a pulse duration of 510 ns (Adrian and Westerweel, 2010). A single cavity of an Nd:YAG laser can be operated at a repetition rate of $10-30 \mathrm{~Hz}$, which matches the frame rate of common dual frame PIV cameras (see Section 3.2.3) (Adrian and Westerweel, 2010). However, as the desired pulse separation is often in the order of microseconds, one must use a dual cavity Nd:YAG laser for PIV systems, by which two separate laser cavities are used to generate the first and second laser pulse. With a dual cavity system the time delay between pulses can be chosen arbitrarily.

A sketch of the main components of a dual cavity Nd:YAG laser is found in Figure 10. Each pumping cavity consists of a laser crystal rod and a linear flash lamp, sealed and surrounded by ceramic reflectors (Raffel et al., 2007). In order to achieve a highenergy pulse with short duration each cavity is equipped with a Q-switch mounted on its backward facing end. The Q-switch consists of a Pockels cell, a $\lambda / 4$ retardation plate, a reflective mirror and a polarizer. Initially the Pockels cell is operated so that light which has been reflected towards the back mirror and passed though the Pockels cell and the $\lambda / 4$ retardation plate twice is rotated $90^{\circ}$ when it returns to the polarizer and therefore rejected by the polarizer (Raffel et al., 2007). At the time of maximum energy storage in the laser rod the voltage to Pockels cell is changed so that it provides a rotation which results in reflected light having the same polarization when it returns to the polarizer. Reflected light then re-enters the pumping cavity, which initiates a strong laser pulse.

In front of the laser cavities is a semi-transparent mirror, followed by a set of mirrors which direct the laser beam on dielectric polarizer that combines the laser beams. The emitted infrared light from the Nd:YAG laser $(1064 \mathrm{~nm})$ is then frequency doubled to visible green light $(532 \mathrm{~nm})$. Additional dichronic mirrors, which have a maximum 
reflectivity at a certain wavelength, are then used to remove light of unwanted frequencies, before the laser light is emitted through the transmitting optics.

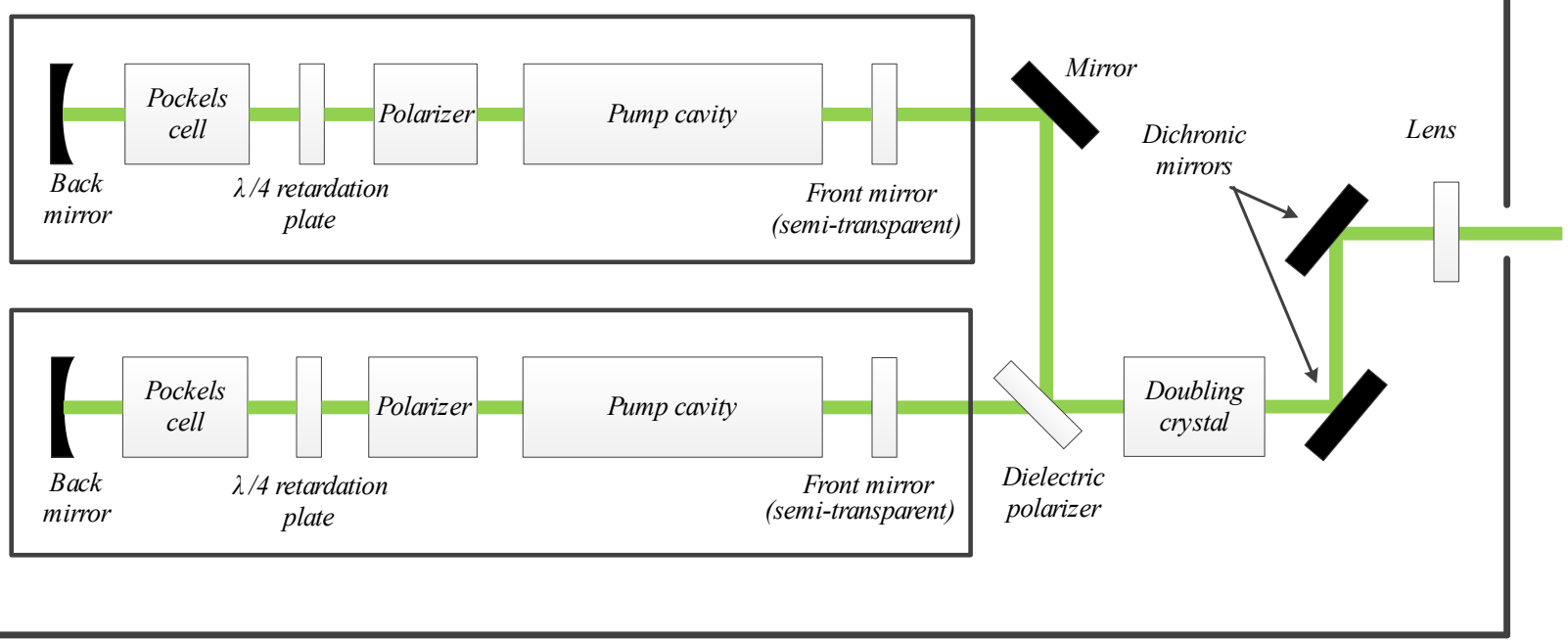

Figure 10 Sketch of main components of dual cavity Nd:YAG laser for PIV.

Then the laser beam is transformed to a laser sheet using a combination of lenses. Most systems use a cylindrical lens with short focal length to expand the beam in one direction (Adrian and Westerweel, 2010). In order to achieve a suitable light sheet thickness a spherical lens may be used after the cylindrical lens. The spherical lens often has a rather long focal length. If a constant width of the light sheet should be obtained, a third cylindrical lens may be employed.

When generating light sheets two aspects are important. First, reflections directed back towards the laser head should be avoided in order not to damage the laser. Secondly, focal points should be avoided as high-intensity laser pulses might ionize the air or burn dust particles at focal points. An example of light sheet optic configuration is found in Figure 11. A description of different setups for light sheet optics may be found in (Raffel et al., 2007).

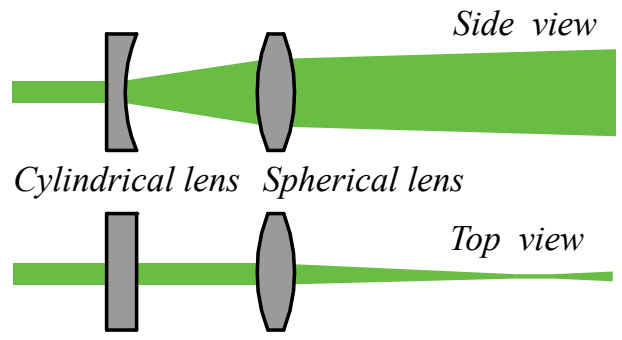

Figure 11 Example of layout of light sheet optics.

\subsubsection{Camera and image acquisition}

Nowadays, digital cameras are most frequently used to capture images in PIV systems. The main component in a digital camera is the imaging sensor, in which the incoming light flux is converted to an electrical signal. For measurements in fluid mechanics based on imaging techniques the imaging sensor is often a CCD (Charged- 
Coupled Device) or a CMOS (Complementary Metal Oxide Semiconductor) (Boutier, 2013).

The imaging sensor of a CCD or CMOS camera consists of an array of small elements, commonly referred to as pixels. Each pixel collects the incoming light and converts it to an electrical signal, proportional to the light flux during the exposure. Modern PIV cameras can contain from 1 million to over 20 million pixels. As the image sensor is read-out, i.e., drained of its electrical change, the amount of collected light in each pixel is reflected as the gray level of that pixel. Tracer particles that are illuminated by the laser sheet, and thereby scatter light, will then appear as bright spots on the resulting image.

The main difference between CCD and CMOS sensors is the read-out procedure. In CMOS cameras each pixel can be controlled and read-out separately. The read-out procedure of a CMOS sensor is fast and the maximum frame rate can be several $\mathrm{kHz}$.

In CCD sensors each pixel stores an electrical charge, corresponding to the received light flux. After the exposure the electrical change of each pixel is successively moved and read-out in sequence on an off-board frame grabber. This read-out procedure results in a typical maximum frame rate of CCD cameras in the order of 2-30 Hz. Generally, higher resolution leads to longer read-out time. Single-exposure, doubleframe, planar PIV requires two separate images to be acquired within a very short time interval. Therefore, special CCD chips, which include a temporary storage of the electrical charge in each pixel from the first exposure, are used in dual frame PIV cameras.

The high frame rate of CMOS sensors makes them suitable for high speed PIV cameras. The downside of CMOS sensors is that they generally have higher noise level and are less sensitive compared to CCD cameras. As transient behavior is not investigated in the present work, a CCD sensor was used in the present investigation.

Tracer particles for PIV measurements may be generated in the same way as for LDA measurements (see Section 3.1.3). However, when the small tracer particles are imaged on an imaging sensor they will appear larger than their physical size due to diffraction and lens abbreviations. If the tracer particle diameter is denoted as $d_{p}$, the resulting diameter on the image $\left(d_{\tau}\right)$ can be approximated according to Eq. (12).

$d_{\tau} \cong \sqrt{M_{0}^{2} d_{p}^{2}+d_{s}^{2}+d_{a}^{2}}$

where $M_{0}$ is the magnification factor, $d_{s}$ the diffraction-limited spot diameter and $d_{a}$ is the aberrated image of a point source.

The diffraction-limited diameter $\left(d_{s}\right)$, which is often the larger term in Eq. (12), (see Adrian and Westerweel (2010) for details), may be calculated according to Eq. (13).

$d_{s}=2.44\left(1+M_{0}\right) f^{\#} \lambda$

$f^{\#}$ is the relative aperture, also called $f^{\#}$-number, of the lens. 
In Eq. (11) a constant magnification factor, $M_{0}$, was used for transforming displacement in the image plane to velocities in the physical plane. However, optical distortions cause inaccuracy by introducing variable magnifications (Soloff et al., 1997). In PIV measurements it is therefore common to use a general mapping function, in combination with one or more calibration images, in order to compensate for variations in magnification at different locations within the field of view.

A calibration image consists of an image taken by the PIV camera, when a plate with markers of known distances is placed in the measurement location, i.e., light sheet location. By knowledge of the distances between the markers a mapping function between image and light sheet plane is calculated. An image mapping function has the general appearance according to Eq. (14).

$X=F(\mathbb{x})$

where $\mathbb{x}=(\tilde{x}, \tilde{y}, \tilde{z})$ is the coordinates in the physical coordinate system, and $\boldsymbol{X}$ is the coordinates in the imaging plane.

A common model for image mapping functions, $\boldsymbol{F}(\mathbb{x})$, proposed by Soloff et al. (1997), is to perform a regression fit to a polynomial with cubic dependence on inplane coordinates and quadratic dependence on the out-of-plane component.

In planar PIV the perspective error due to out-of-plane motion is neglected. A discussion on the effects of possible influence of perspective errors may be found in Raffel et al. (2007). When using a single PIV camera only a single calibration image at $\tilde{z}=0$, i.e., the location of the light sheet is used in the calibration procedure. The present work used the above method, i.e., a cubic mapping function based on a single calibration image.

\subsubsection{Vector calculation and post-processing}

From the two frames of a single PIV recording the instantaneous velocity field is calculated. First the complete field of view is divided into several smaller subdomains, called interrogation areas $\left(A_{I}\right)$. For each interrogation area a cross-correlation is performed which results in a spatial cross-correlation function $R(s)$ of the light intensity between the first and second exposure, according to Eq. (15).

$R(s)=\int_{A_{I}} I_{1}(X) I_{2}(X+s) d X$

where $I_{1}(X)$ is the light intensity of the first exposure, $I_{1}(X+s)$, the light intensity of the second exposure. $s$ is a displacement vector between the two exposures.

A sharp peak in the correlation function, denoted as $R_{d}$, will occur when the second image is translated in such a manner that it overlaps the first. Locations with tracer particles will then coincide and the integrand in Eq. (15) will attain a large value. The values of the displacement vector in which the peak occurs, $s_{D}=\left(S_{X, D}, S_{Y, D}\right)$, correspond to the average particle displacement, $(\triangle X, \triangle Y)$, in the current interrogation area. 
A computationally effective way to compute the cross-correlation function in Eq. (15) is to use the correlation theorem and Fast Fourier Transformation (FFT). This method includes Fourier transformation of the interrogation area in both images. Then a multiplication between the transformation of the first image, $\widehat{I_{1}}$, is multiplied by the complex conjugate of the transformed second image, $\widehat{I}_{2}^{*}$. Finally the crosscorrelation function is held by an inverse transformation of results from the above calculation. Figure 12 shows a sketch of the principal steps of the vector calculation procedure in single-exposure dual frame PIV measurements.

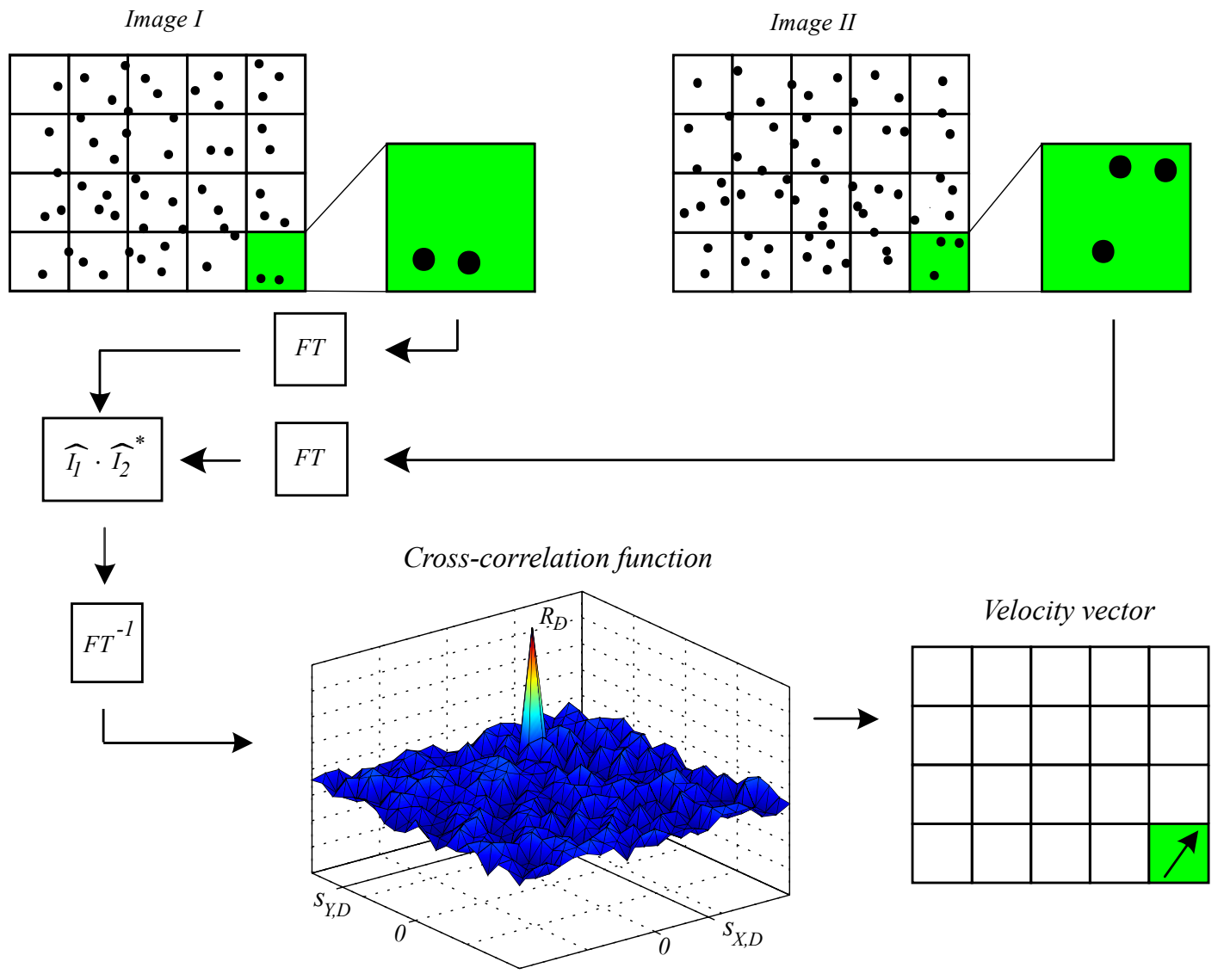

Figure 12 Schematic illustration of main steps in vector calculation in PIV measurements.

Accurate PIV measurements rely on a proper detection of the displacement correlation peak, $R_{D}$. Therefore the optimization of PIV measurements is generally aimed at maximizing the detection probability for $R_{D}$ (Adrian and Westerweel, 2010).

There are several aspects which are important for a successful cross-correlation. One important aspect is the image density $\left(N_{I}\right)$. The image density is defined as the number of particles present in the volume which is bounded by the interrogation area and the light sheet thickness. The image density is calculated according to Eq. (16).

$N_{I}=C A_{I} \triangle \tilde{z}_{0} / M_{0}^{2}$

where $C$ is the mean number of tracer particles per unit volume and $\triangle \tilde{z}_{0}$ is the light sheet thickness. 
The cross-correlation function will integrate over the volume $A_{I} \triangle \tilde{z}_{0}$. If the number of particle pairs which are correlated increases, the height of the correlation peak will increase relative to the increase of noise due to correlation between random particles. It is therefore important to ensure that the image density is not too low.

During the time interval between the first and second exposure particles will both enter and leave the volume $A_{I} \triangle \tilde{z}_{0}$ due to fluid movement. This will result in a loss of particle image pairs that can be correlated. The loss of particle image pairs can be divided into in-plane loss of pairs, where particles leave the interrogation area, and loss of image particle pairs due to out-of-plane movement, where particles are no longer present in the laser light sheet. The degree of lost particle image pairs is commonly described by the fraction of particle image pair that may still be correlated, after losses of image pairs due to fluid movement are accounted for. The factors are denoted as, $F_{I}$ and $F_{O}$, for in-plane and out-of-plane losses respectively. A factor of 1 indicates that there is no loss of particles in the volume $A_{I} \triangle \tilde{z}_{0}$ between exposures. A value of 0 indicated that, within this volume, there are no particles that are present at both exposures.

It is desirable to reduce the loss of particle image pairs, as the loss of particle image pairs will reduce the height of the correlation peak and thereby reduce the measurement accuracy. The in-plane loss of particle pairs will increase when the particle movement increases, as more particles then move out of the interrogation area. However, the in-plane loss of pairs may be minimized $\left(F_{I} \rightarrow 1\right)$ during the vector calculation processing. Two methods for reducing the in-plane loss of pairs are to either use a larger interrogation area in the second image or to use a displacement of the second interrogation area, which corresponds to the movement of the mean flow.

When using advanced PIV processing software the vector calculation is often made through an adaptive multi-pass algorithm. At the first step a large interrogation area, for example $32 \times 32$ pixels, with no window displacement is used. The vector field is calculated and spurious vectors removed. When passed over to the second pass in the multi-pass algorithm, the vector field predicted in the first pass is used as displacement of the interrogation area between exposures in the second pass. In the second pass a smaller interrogation area is used, for example $16 \times 16$ pixels. The process is then repeated on a successively finer grid. Several multi-pass steps may also be performed on the same grid, to gain more accurate displacements of interrogation area, and thereby increase $F_{I}$.

The above procedure, which was also employed in the present work, may both reduce the in-plane loss of pairs and be used to achieve a higher resolution of the vector field, which would not be possible with fixed interrogation areas. Further increase in resolution may be achieved by introducing overlapping interrogation areas.

The out-of-plane loss of pairs increases if the out-of-plane velocity increases or if the light sheet thickness is reduced. The out-of-plane loss of particle image pairs may not be remedied in the post-processing, and must be dealt with when acquiring images. This includes choosing measuring locations with small out-of-plane displacement, or 
incorporating a displacement of the light sheet between the two laser pulses that corresponds to the out-of-plane movement.

In addition to the loss of pairs there are also variations of the velocity within the volume $A_{I} \triangle \tilde{z}_{0}$. As the location of the peak in the cross-correlation function, $R_{D}$, represents a volume average of the movement in this volume, the underlying assumption being that the velocity is constant over $A_{I} \triangle \tilde{z}_{0}$. Variations of velocity within $A_{I} \triangle \tilde{z}_{0}$ result in a broadening of the correlation peak and a reducing maximum value and might eventually lead to failure of a correct detection.

The discrete form of the pixel array in the imaging sensor results in discrete values of the cross-correlation function. This in turn leads to a maximum resolution of \pm 0.5 pixels for $s_{D}=\left(S_{X, D}, S_{Y, D}\right)$. In order to achieve sub-pixel resolution, so-called subpixel estimators are used. A common way to estimate the location of the correlation peak on sub-pixel level, which was used in the present investigation, is to employ a three-point Gaussian fit to the correlation peak value and neighboring pixels. Descriptions of different sub-pixel estimators are found in Raffel et al. (2007).

The particle image diameter, $d_{\tau}$, affects the accuracy of PIV measurements. For small values of, $d_{\tau}$, the particle image will tend to fit into a single pixel only. This leads to a tendency of the displacement peak to attain integer values. This effect is referred to as pixel locking. Raffel et al. (2007) showed that when using a three-point Gaussian subpixel estimator the optimal particle image diameter is approximately 2 pixels.

In addition to the above recommendation, the following four design rules, listed by Adrian and Westerweel (2010), also apply to PIV.

$N_{I}>10$

By keeping the image density above 10 the number of particles is sufficient for a high probability of a valid detection of image displacement.

$\frac{|\triangle z|}{\triangle \tilde{z}_{0}}<0.25$

where $|\Delta z|$ is the particle displacement in the direction normal to the laser light sheet between the exposures, due to out-of-plane movement.

The expression in Eq. (18) represents a rule of thumb for keeping $F_{O}$ above 0.75. As $|\triangle z|$ is proportional to the time delay between laser pulses this design rule sets limits on both $\Delta t$ and light sheet thickness, $\triangle \tilde{z}_{0}$.

$\frac{\triangle X}{A_{I}}<0.25$

The expression in Eq. (19) is similar to Eq. (18) but limits the in-plane loss of pairs, as Eq. (19) is imposed to ensure $F_{I}>0.75$. Using a displacement of the second window, as described earlier, is a common way to circumvent limitations imposed by Eq. (19). 
The final design rule relates to spatial velocity gradients in the interrogation area and states that

$M_{0}|\Delta u| \triangle t<d_{\tau}$

where $|\Delta u|$ is the local variation of velocity in the interrogation area.

The design of the experimental setup in the present work was chosen in order to ensure that the above design rules are generally fulfilled. However, there are regions with either large out-of-plane motion or sharp velocity gradients.

In practical situations the vector calculation will always fail in some of the interrogation areas. What happens in practice is that maximum value in the spatial cross-correlation function, $\mathrm{R}_{D}$, does not relate to the correct displacement. Instead the random noise in $R(s)$ has a peak with higher value, compared to correct displacement peak. The result is a spurious vector in the current interrogation area.

Spurious vectors often occur in regions where the abovementioned design rules are violated or in regions close to solid objects, where laser light reflection might occur. Consequently, the amount of spurious vectors relates to the quality of the PIV measurements and often the percentage of spurious vectors is reported to address measurement quality. Typically 5\% spurious vectors is considered allowable (Adrian and Westerweel, 2010).

Several techniques exist to identify and remove spurious vectors. The present work uses a method where a median test is employed to the vector field. The median test was proposed by Westerweel (1994) and assumes that there is a certain smoothness of the vector field. In principle the median filter works as follows. The velocity in a certain interrogation area, denoted as $U_{i, j}$, is compared to the median of surrounding velocity vectors, $U_{m e d}$. If the residual between the median and the velocity vector exceeds a certain value, the vector is rejected (see Eq. (21)). The threshold for the residual, $\varepsilon_{\text {threshold }}$, may be computed as a factor of the RMS of the vectors used for computing the median. Further development of the median test, with normalization of the residual, was presented by Westerweel and Scarano (2005). As spurious vectors are removed gaps in the vector field are created. These are commonly filled up by interpolation.

$\left|U_{i, j}-U_{\text {med }}\right|<\varepsilon_{\text {threshold }}$

\subsection{Comparison of measurement techniques}

In the present work both LDA and PIV have been used for measurement of the flow field. In this section a short comparison, discussing the strengths and weaknesses of each measurement technique and how the two techniques may complement each other, is provided.

In two aspects the measuring techniques are similar. They are both non-intrusive and do not suffer from the need to place equipment at the location of measurements. The 
downside is that both techniques rely on the use of tracer particles as both PIV and LDA do not actually measure the air velocity but the velocity of the tracer particles.

PIV enables two-dimensional, whole field measurements where instantaneous and mean velocity can be acquired at a large number of points at the same time. From PIV measurements instantaneous velocity derivatives and flow structures may also be identified. However, the accuracy of PIV relates to several factors, such as out-ofplane motion, velocity gradients within the interrogation area, laser sheet reflections from solid objects and laser sheet thickness, to mention a few. While the previously listed design rules give guidance to preferable experimental conditions, these may not always be fulfilled in practice.

LDA is a point-by-point measurement technique, which can make flow field measurements very time-consuming. On the other hand, LDA measurements are known to offer high accuracy and reliability. Measurements with LDA can be taken with a very small measurement volume and may provide high spatial resolution. Thereby the difficulties with measurements in high gradient flows are reduced. As LDA measures each velocity component independently, the problem of out-of-plane motion in planar PIV due to three-dimensional flow field is not present when using LDA. LDA is also a direct measurement technique and does not require calibration toward a known velocity, or as in the case of PIV, a calibration plate. The degree of post-processing is also limited for LDA compared to PIV.

Due to the different strengths and weakness of the two measurement techniques, LDA and PIV may be used to complement each other. PIV is very time-saving when trying to locate characteristic points, such as free stagnation points or local maxima or minima in the flow field. The two-dimensional, whole field measurements also provide more information than a series of single-point measurements. Close to walls or inlets, PIV suffers from both laser reflections and high velocity gradients. Therefore it is often hard to perform measurements in these regions with PIV. With LDA it is generally easier to acquire inlet or boundary layer profiles. Finally, there are a vast number of post-processing options for PIV. LDA data, acquired at a number of sample locations in the region of PIV measurement, is then valuable for comparison with PIV results and for making judgment of suitable processing options. 


\section{Computational Fluid Dynamics}

This chapter gives a short background to Computational Fluid Dynamics (CFD) and strategies for modeling turbulent flows. The emphasis lies on description of basic flow equations and turbulence models that have been used in the CFD simulations in the present work. Different types of boundary conditions are also presented. Finally, numerical aspects such as discretization and convergence are briefly discussed.

As analytical solutions to complex flow field problems are seldom possible, numerical simulations have arisen as an attractive complement to experiments. Detailed experimental campaigns are often both time-consuming and expensive. Computational Fluid Dynamics (CFD) is a collective term for numerical techniques to solve the partial differential equations that govern the flow field development. CFD simulations rely on the equations describing the conservation of mass, momentum and energy. As computer resources are increasing, CFD has gained more interest and CFD simulations are nowadays widely used within several research fields.

Still though, CFD to some extent always relies on a series of modeling assumptions, discretization schemes, meshing strategies, etc. which impose uncertainties on the results. It is therefore important to address the accuracy of CFD simulations. As an example the Journal of Fluids Engineering has developed a set of guidelines in order to address the numerical accuracy in papers considered for publication (Freitas, 1993). The guidelines include requirements on discretization schemes, grid convergence test and, preferably, comparison with experimental data. The latter is generally considered important, especially when a numerical model is employed to a problem where its performance has not been previously evaluated. Even if the need for experimental data often cannot be completely circumvented, CFD simulations serve as a complement to measurements as they provide detailed information of the object of study. CFD simulations result in complete velocity, pressure and temperature fields provided at all locations in the solution domain. CFD not only provides data in locations (or problems) where measurements cannot be performed, but simulation results may also include properties which are difficult to measure, such as dissipation rate or statistics of pressure fluctuations. As boundary conditions and geometry are relatively easy to change in computational models compared to experimental setups, CFD simulations are often a suitable tool for parametrical investigations.

The rapid development of CFD for engineering applications means that nowadays resource-efficient, highly complex and user-friendly CFD software which includes a 
vast number of modeling options is commercially available. CFD software is generally divided into three parts (Versteeg and Malalasekera, 2007).

- Pre-processor

- Solver

- Post-processor

In the pre-processor several steps which relate to the setup of the problem are performed. This includes: specification of the geometry, grid generation (discretization), specification of boundary conditions and material properties, definition of initial solution, convergence criteria and solver settings. Given a complete setup the solver then uses various numerical techniques to solve the system of governing equations, including additional models considering for example turbulence or combustion. With the post-processor, results from the converged solution are extracted and presented in various plots spanning from simple line plots to sophisticated animations.

\subsection{Turbulent flow and modeling strategies}

Turbulent flows are common in engineering applications, such as gas turbines, combustion engines, heat exchangers or ventilation supply devices. A turbulent flow is characterized by the seemingly chaotic and randomly changing flow field. While there may be a steady-state mean velocity or symmetry in the flow field, the random motion in a turbulent flow is unsteady and three-dimensional.

A turbulent flow contains flow structures (eddies) of various sizes and length scales. This results in a transfer of kinetic energy, where large eddies extract energy from the mean flow. The energy is then transferred to smaller and smaller scales, through a process referred to as the cascade process. At very small scales the turbulent kinetic energy is dissipated into heat, due to viscosity. The concept of the cascade process was introduced by Richardson (1922). Kolmogorov (1941) stated that if the Reynolds number is sufficiently high, the statistics of the smallest scales are uniquely determined by the dissipation rate and the viscosity, and there is a region of moderate scales, called the inertial range, where the statistics are determined by the dissipation rate only. The distribution of turbulent kinetic energy on different scales is often analyzed in an energy spectrum, where three regions appear. A sketch of the cascade process and a typical appearance of the energy spectrum are illustrated in Figure 13.

In region $I$ kinetic energy is transferred from the mean flow to large energy-carrying eddies, often in the order of size as for example the jet width itself. In region II, turbulent kinetic energy $(k)$ is transferred to successively smaller eddies, through the cascade process. Region II is characterized by constant transfer of turbulent kinetic energy from region $I$ to the viscous dissipation region (III) and is known for the $-5 / 3$ decay in turbulent kinetic energy in accordance with Eq. (22). In region III the turbulent kinetic energy is converted to heat through viscous dissipation. 
$E(\kappa) \propto \kappa^{-5 / 3}$

where $\mathcal{\kappa}$ is the wave number.

In a spectrum analysis (see Figure 13) it is possible to compute $k$ by integrating the turbulent energy spectrum according to Eq. (23).

$k=\int_{0}^{\infty} E(\kappa) d \kappa$

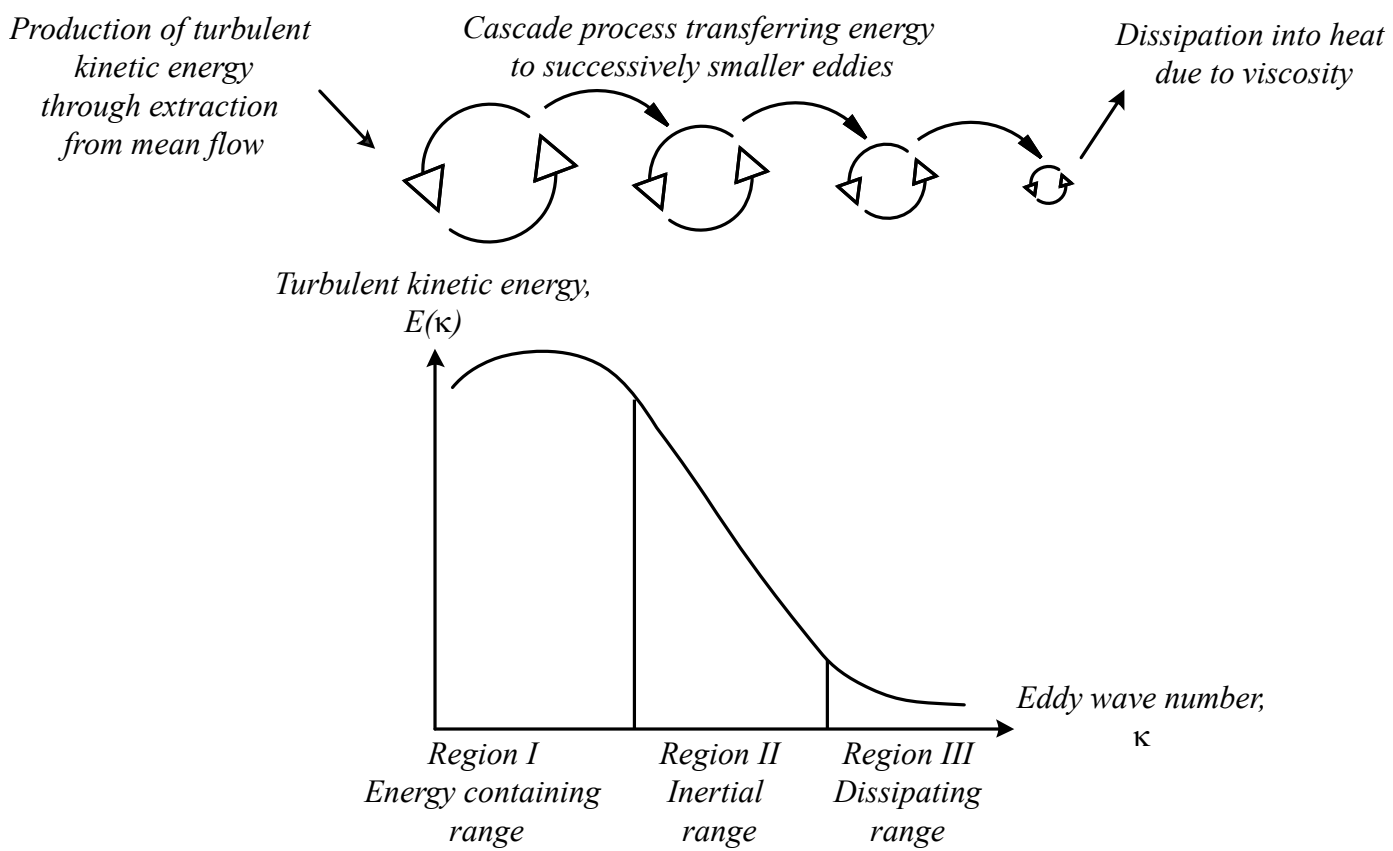

Figure 13 Energy transfer in turbulent flows through cascade process (top) and appearance of the turbulent kinetic energy spectrum (bottom).

From the discussion of the cascade process above another feature of turbulence is established, namely that turbulence is dissipative. In addition to the dissipative nature of turbulence, turbulent flow is also said to be diffusive. Already Reynolds (1883) showed that the occurrence of turbulence greatly enhances mixing due to the random motions in the fluid. An important characteristic of turbulence is therefore its ability to mix fluid more effectively than a comparable laminar flow (Pope, 1978).

Even if turbulent flow is chaotic in nature, the flow is described by a set of conservation laws, including mass, momentum and, in the case of non-isothermal situations, energy. The conservation equations of mass and momentum is referred to as the Navier-Stokes equations. A complete derivation of the Navier-Stokes equations in various forms can be found in Anderson (1995). If the flow is incompressible, Newtonian and without body forces the Navier-Stokes equations are written according to Eq. (24) and Eq. (25).

$\frac{\partial u_{i}}{\partial x_{i}}=0$

$\frac{\partial u_{i}}{\partial t}+\frac{\partial\left(u_{i} u_{j}\right)}{\partial x_{j}}=-\frac{1}{\rho} \frac{\partial p}{\partial x_{i}}+v \frac{\partial^{2} u_{i}}{\partial x_{j} \partial x_{j}}$ 
where $u_{i}$ is the velocity component in the $x_{i}$ direction, $p$ is the static pressure and $v$ the kinematic viscosity.

Eq. (24) describes the conservation of mass in a fluid element and states that the flow field shall be divergence free in an incompressible fluid. Eq. (25) describes the conservation of momentum in each direction. The terms on the left-hand side represent temporal changes in momentum and changes due to convection. The terms on the right-hand side represent the changes in momentum on a fluid element due to pressure gradients and viscous forces.

In the analysis of turbulent flows it is common to divide the instantaneous velocity $\left(u_{i}\right)$ to a fluctuating component $\left(u_{i}^{\prime}\right)$ and a mean velocity $\left(U_{i}\right)$ according to Eq. (26). This decomposition is referred to as Reynolds decomposition.

$u_{i}=U_{i}+u_{i}^{\prime}$

Inserting Eq. (26) to the Navier-Stokes equations (Eq. (24) and (25)) and using Reynolds-averaging for both velocity and static pressure results in the so-called Reynolds-Averaged Navier Stokes equations (RANS) equations presented in Eq. (27) and (28).

$\frac{\partial U_{i}}{\partial x_{i}}=0$

$\frac{\partial U_{i}}{\partial t}+\frac{\partial\left(U_{i} U_{j}\right)}{\partial x_{j}}=-\frac{1}{\rho} \frac{\partial P}{\partial x_{i}}+\frac{\partial}{\partial x_{j}}\left(v \frac{U_{i}}{\partial x_{j}}-\overline{u_{i}^{\prime} u_{j}^{\prime}}\right)$

The turbulent kinetic energy is calculated according to Eq. (29).

$k=1 / 2 \overline{u_{i}^{\prime} u_{i}^{\prime}}$

where the overbar indicates Reynolds-averaged value.

Using the Reynolds decomposition, an exact transport equation for $k$ can be derived according to $\mathrm{Eq}(30)$.

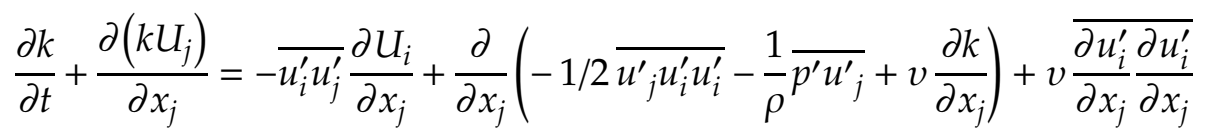

The terms in Eq. (30) has the following physical interpretation

- $\frac{\partial k}{\partial t}+\frac{\partial\left(k u_{j}\right)}{\partial x_{j}}$ represents the temporal change and the convective contribution.

- $\quad-\overline{u_{i}^{\prime} u_{j}^{\prime}} \frac{\partial U_{i}}{\partial x_{j}}$ is referred to as the production term. It is through this term that energy is extracted from the mean flow to large eddies and thereby converted from mean kinetic energy to turbulent kinetic energy. 
- $\frac{\partial}{\partial x_{j}}\left(-1 / 2 \overline{u_{j}^{\prime} u_{i}^{\prime} u_{i}^{\prime}}-\frac{1}{\rho} \overline{p^{\prime} u_{j}^{\prime}}+v \frac{\partial k}{\partial x_{j}}\right)$ relates to turbulent transport, pressure diffusion and molecular diffusion in the order as they appear above.

- $\quad v \overline{\frac{\partial u_{i}^{\prime}}{\partial x_{j}} \frac{\partial u_{i}^{\prime}}{\partial x_{j}}}$ is the rate of dissipation, i.e., the rate of conversion of $k$ to heat (mainly at small scales) per unit time and mass. Commonly the dissipation rate is denoted by the symbol $\varepsilon$.

CFD simulations always rely on the Navier-Stokes equations ((Eq. (24) and (25)), as they represent the conservation laws which the flow field must obey. Using Direct Numerical Simulations (DNS) the Navier-Stokes equations are solved directly. While conceptually simple and unrivalled in accuracy, when applicable such an approach is extremely expensive in terms of computational effort (Pope, 1978). With DNS all turbulent scales are resolved, which requires a very fine grid and short time-step. Due to the high computational effort DNS is therefore not possible in many high Re flows or in situations with complex geometry.

An approach which lies rather close to DNS is Large Eddy Simulations (LES). In LES the Navier-Stokes equations are filtered. Small scales which have a more uniform appearance are modeled through a so-called sub-grid turbulence model, while large, energy-carrying scales that are affected by geometry are resolved (Pope, 1978). The modeling of small scales reduces computational effort compared to DNS, while the challenge of turbulence modeling is still limited as energy-carrying, flow-dependent scales are still resolved. While increasing computational resources are extending the applicability of LES and the use of LES is expected to increase, the method is still considered highly demanding in terms of computational resources.

A less computationally demanding approach is using Reynolds Averaging NavierStokes (RANS) modeling. In RANS modeling, conservation equations are solved for the mean flow, i.e., the RANS equations presented in Eq. (27) and (28). To account for turbulence the mean flow equations are complemented by a turbulence model. In RANS modeling, all scales of turbulence are therefore modeled. While computationally effective, this sets large requirements on the turbulence model. In spite of the rapid development of LES, RANS modeling is still very common for CFD simulations of turbulent flow, especially in complex geometries.

\subsection{RANS turbulence modeling}

The main difference between the RANS equations (Eq. (27) and (28)) and the NavierStokes equations (Eq. (24) and (25)) are the extra terms, $-\overline{u_{i}^{\prime} u_{j}^{\prime}}$, which are referred to as Reynolds stresses.

Using the RANS equations it is possible to simulate the flow field, in terms of mean flow properties, without resolving the instantaneous flow field. The main drawback is that the RANS equations are not a closed equation system, due to the appearance of the Reynolds stresses. Consequently the Reynolds stresses must be modeled using a turbulence model. Different to a sub-grid LES model, a RANS turbulence model 
must include all turbulent scales, which imposes a large challenge on turbulence modeling. A large number of RANS turbulence models exist in the literature. Two common classes of turbulence models are two-equation models, using the Boussinesq approximation, and second-order closure models, often referred to as Reynolds Stress Models (RSM), which solve a separate transport equation for each Reynolds stress. In the present work both second-order closure models and two-equation turbulence models have been employed. The turbulence models used in the present work are described in the following sections. As the CFD simulations are made in steady-state the time-dependent terms are omitted in the model descriptions below.

\subsection{1 $k-\varepsilon$ standard model ( $k-\varepsilon s t d$.}

Two-equation models make use of the so-called Boussinesq approximation, also known as the turbulent-viscosity hypothesis. In linear two-equation models the unknown Reynolds stresses in Eq. (28) are modeled as proportional to the mean flow rate of strain, according to Eq. (31).

$\overline{u_{i}^{\prime} u_{j}^{\prime}}=-2 v_{t} S_{i j}+\frac{2}{3} \delta_{i j} k$

where $S_{i j}$ is the rate of strain tensor defined as

$S_{i j}=\frac{1}{2}\left(\frac{\partial U_{i}}{\partial x_{j}}+\frac{\partial U_{j}}{\partial x_{i}}\right)$

In order to close the equation system the turbulent viscosity, $v_{t}$, must be calculated. In the $k-\varepsilon s t d$. model by Launder and Spalding (1972) the turbulent viscosity is calculated according to Eq. (33).

$v_{t}=C_{\mu} \frac{k^{2}}{\varepsilon}$

where $\varepsilon$ is the dissipation rate of $k$ and $C_{\mu}$ is an empirical model constant.

From the Navier-Stokes equations an exact transport equation for $k$ can be derived (see Eq. (30)). The $k-\varepsilon s t$. uses a modeled transport equation for $k$, given by Eq. (34), which is based to the exact transport equation. In the $k-\varepsilon$ std. model the following assumptions are employed to the transport equations of $k$ : the production term is modeled using the Boussinesq approximation and the turbulent diffusion is approximated with a gradient-diffusion hypotheses.

$$
\underbrace{\frac{\partial\left(k U_{j}\right)}{\partial x_{j}}}_{\text {Convection }}=\underbrace{\frac{\partial}{\partial x_{j}}\left(\left(v+\frac{v_{t}}{\sigma_{k}}\right) \frac{\partial k}{\partial x_{j}}\right)}_{\text {Diffusion }}+\underbrace{2 v_{t} S_{i j} S_{i j}}_{\text {Production }}-\underbrace{\varepsilon}_{\text {Dissipation }}
$$

A transport equation for the dissipation rate is also needed to close the equation system. It is possible to derive an exact $\varepsilon$-equation but it is not useful as a starting point for a modeling equation (Pope, 1978). Instead the $\varepsilon$-equation is based on the assumption that its principal appearance is similar to the modeled k-equation, with 
additional modeling constants added. In order to achieve dimensional correctness the production and dissipation terms are multiplied by the factor $\varepsilon / k$. The resulting transport equation for $\varepsilon$ is found in Eq. (35).

$\frac{\partial\left(\varepsilon U_{j}\right)}{\partial x_{j}}=\frac{\partial}{\partial x_{j}}\left[\left(v+\frac{v_{t}}{\sigma_{\varepsilon}}\right) \frac{\partial \varepsilon}{\partial x_{j}}\right]+2 C_{1 \varepsilon} \frac{\varepsilon}{k} v_{t} S_{i j} S_{i j}-C_{2 \epsilon} \frac{\varepsilon^{2}}{k}$

In the $k-\varepsilon$ std. model the following model constants are used (Launder and Spalding, 1972).

$\sigma_{k}=1.0, \sigma_{\varepsilon}=1.3, C_{1 \varepsilon}=1.44, C_{2 \varepsilon}=1.92, C_{\mu}=0.09$

The $k-\varepsilon s t d$. model is perhaps the least complex, but still complete turbulence model and is incorporated in most commercial CFD software. If used in combination with wall functions (described in Section 4.3) the model is considered easy to use and computationally inexpensive (Pope, 1978). This has led to the $k-\varepsilon s t$. model being the most widely used and validated turbulence model, and its performance has been assessed against a number of practical flows (Tu et al., 2012). The k-E std. model is however also known to produce inaccurate results in several applications. Especially in flows with strong streamline curvature or in boundary layers with strong pressure gradients the $k-\varepsilon s t d$. model may produce faulty results. The model is also known to overpredict the spreading rate of a single round jet. The failure of the $k-\varepsilon$ std. model under certain flow conditions relates to the use of the Boussinesq assumption which does not consider anisotropy in Reynolds stresses and the relatively simple, empirically derived, $\varepsilon$-equation. Several attempts has been made to modify the $k-\varepsilon$ std. model in order to remedy for a particular class of flows (Pope, 1978). For example, adjusting model constants can improve the performance for a single round jet, however at the same time making the turbulence model less general (Tu et al., 2012). Modification of the $\varepsilon$-equation is another approach which has been proposed by several authors.

\subsubsection{RNG $k-\varepsilon$ model}

The RNG $k-\varepsilon$ model is similar to the $k-\varepsilon s t d$. model but is derived from the instantaneous Navier-Stokes equations, using a technique called the renormalization group (RNG) method (Yakhot and Orszag, 1986; Yakhot and Smith, 1992; Yakhot et al., 1992). In the RNG $k-\varepsilon$ model the steady state, incompressible transport equations for turbulent kinetic energy $(k)$ and turbulent dissipation $(\varepsilon)$ are written according to Eq. (36) and (37).

$$
\begin{aligned}
& \frac{\partial\left(k U_{j}\right)}{\partial x_{j}}=\frac{\partial}{\partial x_{j}}\left(\alpha_{k}\left(v+v_{t}\right) \frac{\partial k}{\partial x_{j}}\right)+2 v_{t} S_{i j} S_{i j}-\varepsilon \\
& \frac{\partial\left(\varepsilon U_{j}\right)}{\partial x_{j}}=\frac{\partial}{\partial x_{j}}\left[\alpha_{\varepsilon}\left(v+v_{t}\right) \frac{\partial \varepsilon}{\partial x_{j}}\right]+2 C_{1 \varepsilon} \frac{\varepsilon}{k} v_{t} S_{i j} S_{i j}-C_{2 \epsilon} \frac{\varepsilon^{2}}{k}-\mathscr{R}_{\varepsilon}
\end{aligned}
$$

The additional term $\mathscr{R}_{\varepsilon}$ in the transport equation for dissipation rate, $\varepsilon$, is the main difference between the RNG $k-\varepsilon$ model and the $k-\varepsilon$ std. model and is given by Eq. 
(38). In flows with large strain rate the additional term $\left(\mathscr{R}_{\varepsilon}\right)$ is in the same order of magnitude as the standard term, $C_{2 \epsilon} \frac{\varepsilon^{2}}{k}$ (Smith and Woodruff, 1998).

$\mathscr{R}_{\varepsilon}=\frac{C_{\mu} \eta^{3}\left(1-\eta / \eta_{0}\right)}{1+\beta \eta^{3}} \frac{\varepsilon^{2}}{k}$

where $\eta=\mathscr{S} k / \varepsilon, \eta_{0}=4.38, \beta=0.012$ and $\mathscr{S}=\sqrt{2 S_{i j} S_{i j}}$.

The turbulent viscosity in the RNG $k-\varepsilon$ model is originally computed by a differential equation. According to Yakhot and Orszag (1986) the differential equation will reduce to an algebraic expression for the turbulent viscosity, similar to the $k-\varepsilon$ std. model when the turbulence is fully developed (Eq. (33)).

The constants $C_{\mu}=0.0845, C_{1 \varepsilon}=1.42, C_{\varepsilon 2}=1.68$, and $\alpha_{k}=\alpha_{\varepsilon}=1.39$ are calculated explicitly in the RNG $k-\varepsilon$ model (Yakhot et al., 1992). However, the values are rather similar to the empirical model constants in the $k-\varepsilon s t d$. model. The values of the inverse effective turbulent Prandtl numbers, $\alpha_{k}$ and $\alpha_{\varepsilon}$, are the high Reynolds number limit and according to the RNG $k-\varepsilon$ model the following relationship is used for determining $\alpha_{k}$ and $\alpha_{\varepsilon}$.

$\left|\frac{\alpha-1.3929}{-0.3929}\right|^{0.6321}\left|\frac{\alpha+2.3929}{3.3929}\right|^{0.3679}=\frac{v}{v+v_{t}}$

The RNG $k-\varepsilon$ model has shown improving performance compared to the $k-\varepsilon$ std. model in certain situations, for example in separated flows, such as a backward facing step (Speziale and Thangam, 1992). The RNG $k-\varepsilon$ model is also considered a suitable model for indoor air-flow predictions (Chen, 1995; Chen, 2009). There are also limitations with the RNG $k-\varepsilon$ model. Rokni and Sundén (1999b) and Rokni and Sundén $(1999 \mathrm{a})$ reported that in three-dimensional duct flow, where the $k-\varepsilon s t d$. model fails to predict turbulence driven secondary motions, the use of RNG modelling improve the results with the linear eddy viscosity model (Eq. (31)), but the effect is of less importance than employment of non-linear expressions for the Reynolds stresses. Hanjalić (1999) also stated that in situations where normal straining is dominant, the additional $\mathcal{\varepsilon}$-equation term may even be harmful, as it does not distinguish the sign of the strain $S_{i j}$.

\subsubsection{Reynolds Stress Model (RSM)}

As previously mentioned the usage of the Boussinesq assumption might cause inaccurate behavior in certain situations. Wilcox (1994) lists situations where the Boussinesq assumption fails, which includes flows with sudden changes in mean strain rate, flows over curved surfaces, in ducts with secondary motions or in rotating fluids, three-dimensional flows and flows with boundary-layer separation.

An alternative to the Boussinesq assumption is to derive, and solve, transport equations of the Reynolds stresses. Such an approach will however not close the equation system as the Reynolds stress transport equations will contain third-order correlations. The transport equations for Reynolds stresses must also be 
complemented by a transport equation which relates to a length or time scale of the turbulence, for example the dissipation rate $\varepsilon$ or the specific dissipation rate $\omega=\varepsilon / k$. Even though several terms in the transport equations still require modeling, eliminating the need for using the Boussinesq assumption will remove one of the major drawbacks of the previously discussed two-equation models. Hanjalić (1999) provides a review of situations where RSM shows better results compared to eddyviscosity models, and discusses different modeling challenges when using RSM. The principal appearance and physical meaning of the terms included to the exact transport equations for Reynolds stresses are presented in Eq. (40-46).

$\underbrace{C_{i j}}_{\text {Convection }}=\underbrace{P_{i j}}_{\text {Production }}+\underbrace{D_{L, i j}}_{\begin{array}{c}\text { Molecular } \\ \text { diffusion }\end{array}}+\underbrace{D_{T, i j}}_{\begin{array}{c}\text { Turbulent } \\ \text { diffusion }\end{array}}+\underbrace{\Pi_{i j}}_{\begin{array}{c}\text { Pressure-velocity } \\ \text { gradient tensor }\end{array}}-\underbrace{\varepsilon_{i j}}_{\text {Dissipation }}$

where

$C_{i j}=\frac{\partial\left(U_{k} \overline{u_{i}^{\prime} u_{j}^{\prime}}\right)}{\partial x_{k}}$

$P_{i j}=-\left(\overline{u_{j}^{\prime} u_{k}^{\prime}} \frac{\partial U_{i}}{\partial x_{k}}+\overline{u_{i}^{\prime} u_{k}^{\prime}} \frac{\partial U_{j}}{\partial x_{k}}\right)$

$D_{L, i j}=v \frac{\partial^{2}\left(\overline{u_{i}^{\prime} u_{j}^{\prime}}\right)}{\partial x_{k} \partial x_{k}}$

$D_{T, i j}=-\frac{\partial}{\partial x_{k}}\left(\overline{u_{i}^{\prime} u_{j}^{\prime} u_{k}^{\prime}}\right)$

$\Pi_{i j}=-\frac{1}{\rho} \overline{\left(u_{i}^{\prime} \frac{\partial p^{\prime}}{\partial x_{i}}+u_{j}^{\prime} \frac{\partial p^{\prime}}{\partial x_{i}}\right)}$

$\varepsilon_{i j}=2 v \overline{\frac{\partial u_{i}^{\prime}}{\partial x_{k}} \frac{\partial u_{j}^{\prime}}{\partial x_{k}}}$

The terms in Eq. (41-43), namely convection, production and molecular diffusion, can be computed exactly and require no further modeling.

Often the turbulent diffusion term, $D_{T, i j}$, and the pressure-velocity gradient tensor, $\Pi_{i j}$, are re-written according to Eq.(47). The trace of $\Phi_{i j}$ is zero, which means $\Phi_{i j}$ is a redistributive term. This term, commonly referred to as the pressure-strain term, can be of the same order as the production term $P_{i j}$, and great attention has been paid to modeling of the pressure-strain term. The second part of the pressure-velocity gradient tensor, which is a diffusive term, is generally small and often modeled together with the turbulent diffusion term in Eq. (44). In the present investigation the complete diffusive term $\left(D_{T, i j}^{*}\right)$, including effects from pressure, is modeled according to Eq. (48), as suggested by Lien and Leschziner (1994). 
$\underbrace{-\frac{1}{\rho\left(u_{i}^{\prime} \frac{\partial p^{\prime}}{\partial x_{j}}+u_{j}^{\prime} \frac{\partial p^{\prime}}{\partial x_{i}}\right)}}_{\Pi_{i j}}-\underbrace{\frac{\partial}{\partial x_{k}}\left(\overline{u_{i}^{\prime} u_{j}^{\prime} u_{k}^{\prime}}\right)}_{D_{T, i j}}=$

$\underbrace{\overline{\frac{p^{\prime}}{\rho\left(\frac{\partial u_{i}^{\prime}}{\partial x_{j}}+\frac{\partial u_{j}^{\prime}}{\partial x_{i}}\right)}}-\underbrace{\frac{\partial}{\partial x_{k}}\left(\overline{u_{i}^{\prime} u_{j}^{\prime} u_{k}^{\prime}}+\frac{\overline{p^{\prime} u_{i}^{\prime}}}{\rho} \delta_{j k}\right.}_{D_{T, i j}^{*}}+\overline{p^{\prime} u_{j}^{\prime}} \delta_{i k})}_{\Phi_{i j}}$

$D_{T, i j}^{*}=\frac{\partial}{\partial x_{k}}\left(\frac{v_{t}}{\alpha_{k}} \frac{\partial\left(\overline{u_{i}^{\prime} u_{j}^{\prime}}\right)}{\partial x_{k}}\right)$

Several different variants of pressure-strain models exist. The pressure-strain term is often decomposed in three parts according to

$\Phi_{i j}=\Phi_{i j, 1}+\Phi_{i j, 2}+\Phi_{i j, w}$

where $\Phi_{i j, 1}$ is the slow pressure-strain term, $\Phi_{i j, 2}$ is the rapid pressure-strain term and $\Phi_{i j, w}$ is the wall reflection or wall echo term.

Launder (1989a), Launder (1989b) and Gibson and Launder (1978) proposed a linear pressure strain model where the three contributions to the pressure-stain terms are modeled according to Eq. (50-52). It can be noted that several proposals exist for both the slow, but above all the rapid term.

$$
\begin{aligned}
& \Phi_{i j, 1}=-C_{1} \frac{\varepsilon}{k}\left(\overline{u_{i}^{\prime} u_{j}^{\prime}}-\frac{2}{3} \delta_{i j} k\right) \\
& \Phi_{i j, 2}=-C_{2}\left[P_{i j}-C_{i j}-\frac{1}{3} \delta_{i j}\left(P_{k k}-C_{k k}\right)\right] \\
& \Phi_{i j, w}=C_{1}^{\prime} \frac{\varepsilon}{k}\left(\overline{u_{k}^{\prime} u_{m}^{\prime}} n_{k} n_{m} \delta_{i j}-\frac{3}{2} \overline{u_{k}^{\prime} u_{i}^{\prime}} n_{k} n_{j}-\frac{3}{2} \overline{u_{k}^{\prime} u_{j}^{\prime}} n_{k} n_{i}\right) f_{w} \\
& +C_{2}^{\prime}\left(\Phi_{k m, 2} n_{k} n_{m} \delta_{i j}-\frac{3}{2} \Phi_{i k, 2} n_{k} n_{j}-\frac{3}{2} \Phi_{j k, 2} n_{k} n_{i}\right) f_{w}
\end{aligned}
$$

where $\boldsymbol{n}$ is a unit vector normal to the wall.

The near-wall function $f_{w}$ is modeled as

$f_{w}=\frac{C_{\mu}^{3 / 4}}{\kappa} \frac{k^{3 / 2}}{\varepsilon d}$

where $\kappa$ is the von Kármán constant $(0.4187)$ and $d$ the distance to the wall.

The model constants in the linear pressure-strain term are:

$C_{1}=1.8, C_{2}=0.6, C_{1}^{\prime}=0.5, C_{2}^{\prime}=0.3$ 
The pressure-strain model described above, which exists in different variants, is often referred to as the IP model. The model is presented in detail by Launder and Sandham (2002).

Dissipation occurs at the smallest scales, which according to the hypothesis by Kolmogorov (1941) are isotropic. Therefore most modelers apply the following relationship for the dissipation rate, where the scalar quantity $\varepsilon$ is the dissipation rate which appears in the transport equation for turbulent kinetic energy (Wilcox, 1994).

$\varepsilon_{i j}=\frac{2}{3} \varepsilon \delta_{i j}$

where the transport equation for $\varepsilon$ in the RSM has the following form:

$\frac{\partial\left(\varepsilon U_{i}\right)}{\partial x_{i}}=\frac{\partial}{\partial x_{j}}\left[\left(v+\frac{v_{t}}{\sigma_{\varepsilon}}\right) \frac{\partial \varepsilon}{\partial x_{j}}\right]-C_{1 \varepsilon} \overline{u_{i}^{\prime} u_{j}^{\prime}} \frac{\partial U_{i}}{\partial x_{j}} \frac{\varepsilon}{k}-C_{2 \epsilon} \frac{\varepsilon^{2}}{k}$

where $C_{1 \varepsilon}=1.44, C_{2 \varepsilon}=1.92$ and $\sigma_{\varepsilon}=1.3$.

\subsection{Boundary conditions}

For a CFD simulation to be successful appropriate boundary conditions must be applied at all boundaries of the computational domain. Frequently used boundary conditions are:

- Inlet

- Outlet or prescribed pressure

- Symmetry

- Wall

The inlet represents a location where mass and momentum is added to the computational domain. In RANS modeling an inlet requires specification of both mean and turbulence properties. For two-equation $k-\varepsilon$ models or RSM using the $\varepsilon$ equation, turbulent inlet properties may be defined by specifying turbulent kinetic energy or Reynolds stresses and approximating the dissipation rate according to Eq. (56).

$\varepsilon=\frac{C_{\mu}^{3 / 4} k^{3 / 2}}{l}$

where $l$ is the turbulent length scale at the inlet, often approximated as $l=0.07 d_{0}$.

As an alternative to the above, turbulence intensity $\left(T_{U}\right)$ may also be used for specifying boundary conditions. In that case the turbulent kinetic energy at the inlet is computed according to Eq. (57). 
$k=\frac{3}{2}\left(U_{b} \cdot T_{U}\right)^{2}$

where $U_{b}$ is the mean inlet velocity

For the RSM the Reynolds stresses are then approximated based on the assumption that the turbulence is isotropic. The dissipation rate is computed in the same manner as when specifying $k$ directly (Eq. (56)).

For boundaries where fluid exits the computational domain, two different approaches exist. One approach is to assume that the outlet is placed far enough in the downstream direction so that the flow field is fully developed, i.e., gradients of all properties (except pressure) are assumed to be zero in the direction normal to the boundary (Versteeg and Malalasekera, 2007). Alternatively outflow boundaries may be employed with a constant static pressure, commonly referred to as prescribed pressure boundary condition or pressure outlet. As backflow, i.e., fluid entering the domain, can occur at a prescribed pressure boundary turbulent properties must be specified in the same manner as for inlets.

If the computational domain has a symmetric appearance symmetry boundary conditions can be used to reduce the computational domain size, and thereby the required computational effort. At symmetry boundary conditions the velocity in the direction normal to the boundary and gradients of all variables in the normal direction are set to zero according to Eq. (58).

$U_{n}=\frac{\partial \phi}{\partial n}=0$

where $n$ represents the wall normal direction.

As the flux per definition is zero across a symmetry boundary condition and since the shear stress is also zero at a symmetry boundary, the symmetric boundary condition may also be interpreted as a "slip" wall in viscous flow calculations (ANSYS Inc., 2012).

Generally, a no-slip condition is considered as the wall boundary condition. However, in the vicinity of walls the flow is highly complex. Near-wall effects such as high shear rate, two-component turbulence and wall blocking necessitate modifications to the basic $k-\varepsilon$ and RSM models (Pope, 1978).

It has been shown that in the near-wall region turbulence models based on the $\varepsilon$ equation cannot be used to resolve the turbulent flow field without modifications. In the wall function approach introduced by Launder and Spalding (1972) the concept is that instead of resolving the wall boundary layer all the way to the viscous sub-layer the first computational node is placed in the logarithmic layer. Wall functions are then used to bridge the near wall behavior to the first computational node. In other words the computational nodes next to the walls are used as a "matching point" with the law of the wall. 
The wall boundary conditions, using the standard wall function approach proposed by Launder and Spalding (1974), are implemented as follows. The subscript $p$ denotes the location of the node next to the wall. The velocity in the direction along the wall is computed according to the law of the wall (Eq. (59)).

$U^{*}=\frac{1}{\kappa} \ln \left(E y^{*}\right)$

where $\mathrm{E}$ is an empirical constant $(=9.793)$.

The dimensionless velocity and wall distance are computed according to:

$U^{*}=\frac{U_{P} C_{\mu}^{1 / 4} \sqrt{k_{P}}}{\tau_{w} / \rho}$

$y^{*}=\frac{C_{\mu}^{1 / 4} \sqrt{k_{p}} y_{P}}{v}$

where $\tau_{w} / \rho=\sqrt{C_{\mu}} k$

If the first node is located below $y^{*}<11$ the velocity is calculated according to $U^{*}=y^{*}$ instead of Eq. (59).

At the wall node local equilibrium between production of $k$ and its dissipation rate $\varepsilon$ is assumed. This yields the boundary condition for the dissipation rate according to Eq. (62).

$\varepsilon_{P}=\frac{C_{\mu}^{3 / 4} k_{P}^{3 / 2}}{\kappa y_{P}}$

For the turbulent kinetic energy a zero gradient is employed in the direction normal to the wall.

$\frac{\partial k}{\partial n}=0$

where $n$ represents the wall normal direction.

When the RSM is used wall boundary conditions are needed for the individual stresses. Using perturbation methods it is possible to derive a relationship for the Reynolds stresses in the wall adjacent nodes, which has the following general form (Wilcox, 1994).

$\overline{u_{i}^{\prime} u_{j}^{\prime}}=W_{i j} k \quad$ as $y \rightarrow 0$

$W_{i j}$ is a matrix of coefficients using a coordinated system which is aligned with the wall. The precise form of $W_{i j}$ depends on the closure coefficients used. 
The standard wall function approach is based on several assumptions and its accuracy depends on the validity of the wall functions. Not only does this restrict the situations where this approach may produce accurate results. It also imposes requirements on the location of the first grid point, which must be placed in the logarithmic layer. If the grid is refined so that the first computational grid point is placed in the viscous sub-layer $\left(y^{*}<11\right)$ the solution might become dependent on grid resolution (wall distance).

As an alternative to standard wall functions, the turbulence model may be complemented with different types of near-wall treatments. There are several approaches in which the boundary layer is resolved all the way down to the viscous sub-layer. One possible way is to complement the turbulence model with damping functions that modify the behavior of the turbulence model close to the walls. There are also models which employ a so-called two-layer, or zonal approach, where a simpler model such as the one-equation model by Wolfshtein (1969) is employed in the viscous affected wall region. There is also the class of turbulence models which uses $\omega=\varepsilon / k$ as scale equation instead of the $\varepsilon$-equation. The $\omega$-equation may be integrated directly through the viscous sub-layer. For further reading regarding nearwall treatment see Wilcox (1994).

All the above alternatives involve resolution of the viscous sub-layer, which may substantially increase the required number of grid points, and thereby the required computational effort. A slightly different approach is to use the scalable wall functions proposed by Grotjans and Menter (1998). The scalable wall functions are very similar to the standard wall functions but contain a limiter which forces the use of the logarithmic law, by placing the first grid point at the edge of the viscous sublayer. Thereby the scalable wall functions are consistent during grid refinement. The additional limiter for the scalable wall function is presented in Eq. (65).

$y^{*}=\max \left[\frac{C_{\mu}^{1 / 4} \sqrt{k_{p}} y_{P}}{v}, 11\right]$

\subsection{Numerical aspects}

CFD involves using numerical techniques to solve the partial differential equations that govern the flow field. Most commercial CFD solvers use a method called Finite Volume Method (FVM). In the FVM the Navier-Stokes equations are solved in their integral form over a large set of small control volumes. The FVM includes the following three steps (Versteeg and Malalasekera, 2007):

1) Integration of governing equations in all control volumes.

2) Conversion of integral equations to algebraic set equations, using discretization.

3) Solving of algebraic equations using an iterative process. 
All three steps have implications on solution accuracy. In the first step, integration, the Gauss divergence theorem is used to convert the volume integrals in the conservation equations to surface integrals. The integration over the control volume in the FVM results in conservation of all relevant parameters within each control volume. In order to resolve the flow field the size of the control volumes must not be too large. Especially in regions with high gradients the mesh resolution must be made fine enough to capture the flow field correctly. At the same time the required computational effort increases with grid resolution. Mesh generation is therefore often a trade-off between required accuracy and computational effort.

The common way to ensure sufficient grid resolution is to gradually refine the mesh and evaluate the change in solution. When only insignificant change in solution during refinement remains, the solution is said to be grid independent. A detailed discussion on grid resolution, including formal methods for evaluating grid independence are found in Roache (1997).

The second part, discretization, is an important step. Errors due to discretization are one of the main error sources in CFD. Generally a discretization scheme should fulfill the following three fundamental properties: conservativeness, boundedness and transportiveness (Versteeg and Malalasekera, 2007). In summary this implies that the discretization scheme should:

a) result in consistent values on shared faces between two adjacent cells.

b) result in nodal cell values which are limited by the values of surrounding cell faces (provided that no source terms are present).

c) reflect the directional dependence in situations with considerable convection.

In addition to the above the discretization scheme should also preferably have a high order of accuracy to minimize discretization errors and not introduce so-called false diffusion, i.e., tend to smear out gradient in an unphysical manner.

For the diffusion (viscous) terms the second-order accurate central differencing scheme is often used, as also in the present study. For the convection terms, the central differencing scheme lack transportiveness, and therefore some kind of upwind biasing is generally introduced in order to meet criteria c). Also, central order differencing has been shown to cause wiggles (violating criteria b)) in situations where convection is dominant.

The first-order upwind scheme provides a stable discretization scheme for the convection terms, which meets all three criteria listed above. However, the scheme is only first-order accurate, and may introduce considerable false diffusion, especially if the cell orientation is not aligned with the flow direction. To avoid false diffusion, and to achieve a higher order of accuracy, higher order discretization schemes such as the second-order upwind scheme or the third-order accurate QUICK scheme may be used for the convective term. Using the second-order upwind scheme may reintroduce wiggles in the flow field due to un-bounded solutions in regions with high gradients (Anderson, 1995). To overcome this problem with the second-order upwind 
scheme, flux or gradient limiters are introduced, which ensures boundedness of the second-order upwind scheme.

After discretization the governing differential equations are represented by a large set of algebraic equations, which may be solved using an iterative process. A common approach for incompressible flows, used in the present work, is the Semi-Implicit Method for Pressure-Linked Equations (SIMPLE) algorithm, which was proposed by Patankar and Spalding (1972). The SIMPLE algorithm start with an initial guess of velocities, pressure and other scalars included to the setup. In the second step the discretized momentum equations are solved. A pressure correction function, based on the continuity equation, is then solved and provides a correction on the pressure field in order to better fulfill the continuity condition. The initial guesses of velocity and pressure field are then updated based on the pressure correction. Finally, all other scalar transport equations are solved, and values of scalars are updated. The updated values of all variables are then used as initial guess in the next iteration.

To prevent the iterative process from diverging, under-relaxation factors are generally applied when updating variables. The choice of under-relaxation factors affects both convergence and computational effort. Too high values of under-relaxation factors (close to unity) may cause the solution to diverge, while decreasing the underrelaxation factors too much leads to an increasingly slow progression of the flow field in the solution domain.

A common way to assess the convergence is monitoring of the residuals. The residuals are a measure of the imbalance of different variables in each cell, summed over all cells in the computational domain. In order to allow evaluation of the residuals' relative size, the residuals are often scaled in a manner which relates to the absolute values of the variable throughout the computational domain.

If the solution is converging the residuals should be reducing. When the residuals of all variables falls below a certain, pre-defined value, the solution is considered converged. This type of criterion often constitutes the basis for judging convergence. However, the values and behavior of residuals depend on several factors, such as initial solution, grid resolution and under-relaxation factors. Therefore, it is important to also ensure that the solution has stabilized, for example by evaluating the velocity in a set of locations within the solution domain. A combination of low residuals (which are not slowly increasing) and a fully developed and stabilized solution is a more complete convergence criterion than just judging residual values alone. 


\section{Statistical methods}

This chapter gives an introduction to the statistical methods used in the present work. The chapter starts with a discussion on parametrical investigations in general. Then the concept of Response Surface Methodology is explained and different methods related to Design of Experiments (DoE) are presented. The chapter ends with a discussion regarding validation of derived response surface models.

There are numerous scientific and engineering problems which have been subject to parametrical investigations. A parametrical investigation basically means that one wishes to investigate how one or several dependent variables (response variables) depend on a set of independent variables (factors). There are several reasons why parametrical investigations are conducted. For example, the investigator might be interested in knowing what factors are the most influential on certain response variables, what values of different factors will result in a certain response variable value, or how the response variable could be optimized, i.e., maximized or minimized.

A primitive way of conducting parametrical investigations is the best-guess approach. The best-guess approach starts with a single test, where a combination of factors is employed as an initial guess. If the desired output is not achieved, the factor values are tweaked based on some idea of how the system responds, and the test is repeated. If the desired conditions are still not met, the process is repeated again in order to improve the response. The success of this method relies on experience in how the system behaves. As stated by Montgomery (2012) this method often works fairly well in practice, but has the disadvantage that there is no way to know in advance how many tests are needed before the goal is met, if it is ever achieved at all. Nor is there any way to know when an optimal solution is achieved, or even get an approximation of how far from an optimized solution the current results are.

Considering more structured ways for conducting parametrical investigations there are two principally different approaches:

- The one-factor-at-a-time approach.

- The multiple-variable (factorial) approach.

With the one-factor-at-a-time approach the value of one factor at a time is varied while the other are kept fixed. The main disadvantage of this method is that it fails to consider any interactions between factors (Montgomery, 2012). With a factorial approach the factors are varied simultaneously, which means that interactions between factors are also considered. Also, if one aims to investigate more than one 
factor, the one-factor-at-a-time approach is less efficient compared to a factorial approach.

\subsection{Response Surface Methodology}

Response Surface Methodology is a set of mathematical and statistical methods used to construct models that predict the values of one or several response variables, given a combination of factors. The method to some extent always relies on fitting approximate functions to a number of points within the design space (design points), where the values of the response variables are known. The method was introduced by Box and Wilson (1951) and has proven to be an efficient way of studying complex systems.

Historically, physical experiments were used to provide data of response variables at the design points. However, as numerical simulations are becoming increasingly common within both engineering and scientific investigations, design point data is nowadays also acquired from numerical simulations. Examples of investigations that combine CFD and Response Surface Methodology are the studies by Chen et al. (2013) and Norton et al. (2010) who studied ventilation in offices and livestock buildings respectively, and Larraona et al. (2013) who investigated the electronic cooling performance by means of an impingement jet in a cross-flow.

In Response Surface Methodology the response variable $Y$ is assumed to have the following relationship with $k$ number of factors, denoted as $x_{1}, x_{2}, x_{3}, \ldots, x_{k}$.

$Y=f\left(x_{1}, x_{2}, x_{3}, \ldots, x_{k}\right)+\varepsilon$

where $f$ is an unknown response function and $\varepsilon$ is an error term, due for example to measurement errors, not reproduced by $f$.

As $f$ is unknown a common approach in Response Surface Methodology is to use a low order polynomial function, fitted to a number of points in the design space, as approximate function to $f$. Using a second-order polynomial the approximate function has the following appearance.

$Y=\beta_{0}+\sum_{i=1}^{k} \beta_{i} X_{i}+\sum_{i=1}^{k} \beta_{i i} X_{i} X_{i}+\sum_{i=1}^{k} \sum_{j \geq i}^{k} X_{i} X_{j}+\varepsilon$

where $X_{i}$ is the input factors as coded variables (see Section 5.2) and $\beta_{0}$, $\beta i$ and $\beta_{i j}$ are unknown model coefficients.

If the system under consideration has three factors Eq. (67) contains one constant term, three linear terms, often referred to as main effects, three quadratic terms and three interaction terms between different factors. All terms have an unknown coefficient $\left(\beta_{0}, \beta_{i}\right.$ or $\left.\beta_{i j}\right)$. The coefficient values can be determined through leastsquares approximation, once the response variable values have been acquired at a number of design points. The number and location of design points is a very 
important aspect of Response Surface Methodology and is generally referred to as Design of Experiments (DoE).

\subsection{Design of Experiments (DoE)}

The DoE strategies were originally developed for fitting approximate models to data taken from physical experiments, but may also be applied to results from numerical simulations (Alvarez, 2000).

A proper DoE greatly facilitates fitting and analysis of Response Surface (RS) models (Montgomery, 2012). Box and Draper (1987) concluded that the aims when selecting an experimental design are multifaceted and also presented a list of desirable properties for an appropriate DoE, which includes: generate a satisfactory distribution of information (design points) throughout the region of interest; ensure that the fitted value be as close as possible to the true value; provide an internal estimate of error; and require a minimum number of experimental runs.

In DoE it is suitable to transform natural variables into coded variables, $X_{i}$, which takes values between $-1 \leq X_{I} \leq 1$, as coded variables are effective in determining the relative size of factor effects (Montgomery, 2012). The transformation from natural to coded variables is described by Eq. (68).

$X_{i}=2 \frac{x_{i}-x_{i M}}{x_{i H}-x_{i L}}$

where $X_{i}$ and $x_{i}$ represent the $i$ th factor as coded and natural variables, respectively, $x_{i L}$ and $x_{i H}$ represent the low, and high levels of the $i$ th natural variable and $x_{i M}$ is their mean value.

Consider an RS model consisting of a second-order polynomial, such as the one in Eq. (67). A proper fit of this model requires at least three different levels of each factor. A straightforward DoE is defining the design points as all possible combinations where the coded factors have values of $-1,0$ or 1 . This is called a full factorial design. An example of a full factorial design with three factors is shown in Figure 14. If no runs are repeated a full factorial design with three factors requires 27 runs. At the same time there are only 10 coefficients to be determined in the RS model. This means that for this example the full factorial design has a redundancy factor, defined as the number of runs divided by the number of model coefficients, of $27 / 10=2.7$.

The number of runs in a full factorial design with three levels increases by $3^{k}$, where $k$ is the number of factors. Therefore this design is often referred to as $3^{k}$ factorial design. In Table 5 the number of runs and the number of coefficients to be determined are listed for an increasing number of factors.

The $3^{k}$ factorial design rapidly leads to a very large number of runs. At the same time the redundancy factor increases for larger number of factors. As experiments or numerical simulations can be both time-consuming and expensive there is often a 
need to reduce the number of runs through a so-called fractional factorial design, which has lesser number of runs, and thereby a redundancy factor closer to unity.

There are several variants of fractional factorial designs (see for example Khuri and Cornell (1996)). Two commonly used classes of quadratic fractional factorial designs are the Central Composite Design (CCD) and the Box-Behnken Design (BBD). In Figure 14 an example of the principal distribution of runs within the design space, consisting of three factors, is shown for the two classes of fractional factorial designs. The required number of runs, without any repeated runs, is presented in Table 5 for different number of factors.

Table 5 Required number of runs for different three level (full and fractional) factorial designs.

\begin{tabular}{ccccc}
\hline & Number of runs \\
$\begin{array}{c}\text { Number } \\
\text { of } \\
\text { factors }\end{array}$ & $\begin{array}{c}\text { Number of } \\
\text { coefficients in } \\
\text { quadratic } \\
\text { model }\end{array}$ & $\begin{array}{c}3^{k} \text { full } \\
\text { factorial } \\
\text { design }\end{array}$ & $\begin{array}{c}\text { Central Composite } \\
\text { Design (CCD) } \\
\text { (Box and Wilson, 1951) }\end{array}$ & $\begin{array}{c}\text { Box-Behnken Design } \\
\text { (BBD) }\end{array}$ \\
\hline 2 & 6 & 9 & 9 & $1960)$ \\
3 & 10 & 27 & 15 & - \\
4 & 15 & 81 & 25 & 25 \\
\hline 5 & 21 & 243 & 43 & 41 \\
6 & 28 & 729 & 77 & 61 \\
7 & 36 & 2187 & 143 & 85 \\
\hline
\end{tabular}

Several variants of the CCDs exist, but generally a CCD consists of $2^{k}$ points that form the design space "cube," $2 k$ axial points and a number of repeated center points, $C_{0}$, the center point being the design point located at the center of the design space. The total number of runs in a three-level CCD is therefore $2^{k}+2 k+C_{0}$.

The example in Figure 14 is a design called Face Centered, where the axial points, positioned at $[\alpha, 0,0],[0, \alpha, 0]$ and $[0,0, \alpha]$, are located at $\alpha= \pm 1$. The value of $\alpha$ however varies between different designs to hold certain design properties, such as rotatability or orthogonal blocking. Discussion of these properties and the choice of $\alpha$ using CCD may be found in for example Box and Draper (1987).

The Box-Behnken design was introduced by Box and Behnken (1960). An advantage of the Box-Behnken design is that it avoids combinations of extreme values of factors, i.e., the "corner" points in the design space cube in Figure 14, which might be favorable when conducting experiments. The total number of design points in a BBD is $2 k(k-1)+C_{0}$, where $k$ is the number of factors and $C_{0}$ the number of center point runs. 

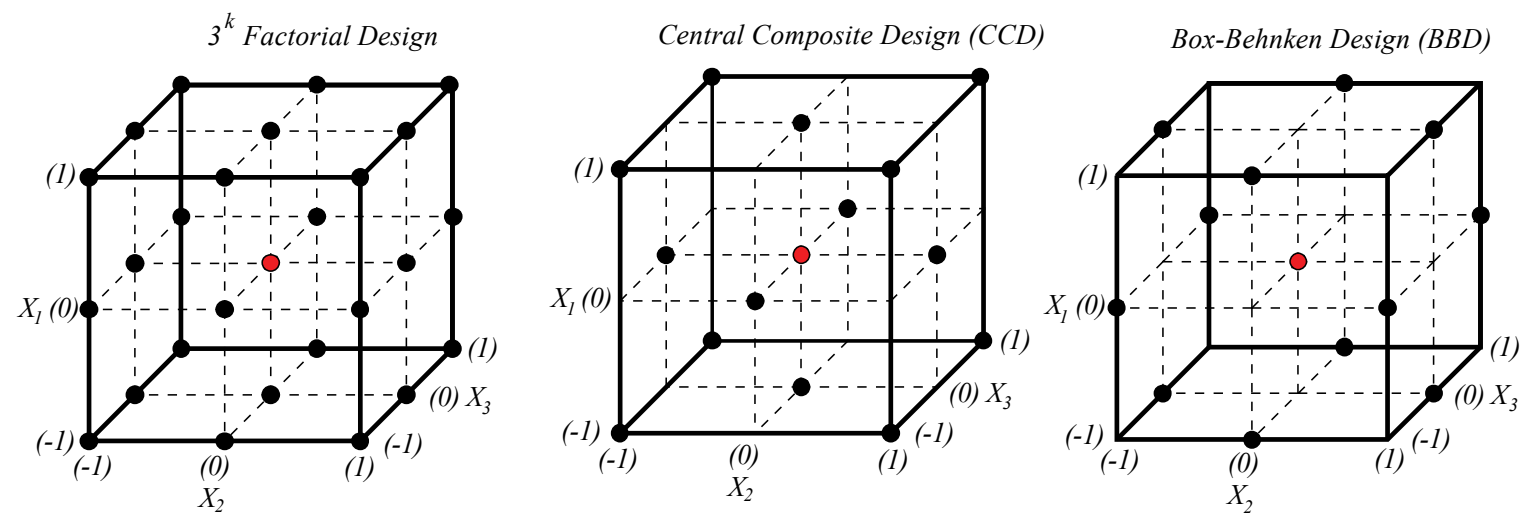

Figure 14 Distribution of runs (data points) in different factorial designs.

The required number of runs with different designs, without additional center point runs, for increasing number of factors is presented in Table 5. A comparison of the efficiency of CCD and BBD designs was performed in Ferreira et al. (2007). It was concluded that $\mathrm{BBD}$ was slightly more efficient compared to the $\mathrm{CCD}$ and much more efficient than the three-level full factorial design.

The number of repeated center point runs is a parameter to be determined within the DoE. The inclusion of multiple center point runs relates to the wish to separate the total lack of fit in experimental uncertainties and lack of fit of the model itself, due for example to higher order terms. Repeating the center point run a number of times while using a single run in all other design points offers an independent estimate of pure experimental error, while maintaining model orthogonality (Khuri and Cornell, 1996).

The method described above is intended for design point data acquired through experiments. Numerical simulations will reproduce the same results with insignificant variation if the same simulation is repeated, therefore there is no need to perform more than one simulation of the central point when design point data is derived from numerical simulations (Larraona et al., 2013; Norton et al., 2010).

\subsection{Validation of the RS model}

After the RS model coefficients have been derived, using least-square approximation, the adequacy of the RS model in terms of representing true relationships between included factors and response variables, as well as ability to accurately reproduce response variable values, must be addressed.

The first step is to investigate whether the included terms in the RS model have statistically significant influence on the response variables. This is commonly made through a so-called analysis of variance (ANOVA) test.

The idea behind the ANOVA test is that the total variability in the data can be divided in two parts. The first part is the variability between factor level mean values and the grand mean of the data. The second part is the sum of the variability within each factor level compared to the mean of the observations at the factor level under consideration. The above reasoning may be written according to Eq. (69). 


$$
\sum_{i=1}^{a} \sum_{j=1}^{n}\left(y_{i j}-\bar{y}\right)^{2}=n \sum_{i=1}^{a}\left(\overline{y_{i}}-\bar{y}\right)^{2}+\sum_{i=1}^{a} \sum_{j=1}^{n}\left(y_{i j}-\overline{y_{i}}\right)^{2}
$$

where $y_{i j}$ is the $j$ th observation at the $i$ th factor level, $a$ is the number of factor levels and $n$ is the number of observations within each factor level.

The grand mean value, $\bar{y}$ and the mean values of each factor level, $\overline{y_{i}}$ is computed according to Eq. (70) and Eq. (71) respectively.

$\bar{y}=\frac{1}{a \cdot n} \sum_{i=1}^{a} \sum_{j=1}^{n} y_{i j}$

$\overline{y_{i}}=\frac{1}{n} \sum_{j=1}^{n} y_{i j}$

The hypothesis that there is no difference between factor level mean values $\overline{y_{i}}$, can be tested by performing an F-test according to Eq. (72) (see for example Montgomery (2012) for details).

$F_{0}=\frac{\left[\frac{n \sum_{i=1}^{a}\left(\overline{y_{i}}-\bar{y}\right)^{2}}{(a-1)}\right]}{\left[\frac{\sum_{i=1}^{a} \sum_{j=1}^{n}\left(y_{i j}-\overline{y_{i}}\right)^{2}}{a(n-1)}\right]}$

The hypothesis that the factor level mean values are equal, meaning that the factor under consideration has no influence, may be rejected if the resulting $F_{0}$ is larger than a certain threshold, for an $F$-distribution with $a-1$ and $a(n-1)$ degrees of freedom. Alternatively one may also use the $P$-value in the above test. If $P<0.05$ one may reject, on a $95 \%$ confidence interval, that the factor level mean values are equal. The ANOVA test can be extended to include interaction terms as well as quadratic terms.

Based on the results from the ANOVA test the final RS model is generated in an iterative manner where statistically insignificant terms $(P>0.05)$ are successively removed and the RS model recomputed until only significant terms remain.

At the end, the accuracy of the final RS model is addressed by computing the $R^{2}$ value or adjusted $R^{2}$-value in the design data points. An $R^{2}$-value that approaches $100 \%$ indicates that the variability of the response variable within the design space is to a large degree captured by the RS model.

Further analysis of model adequacy can be performed by various means of graphical analysis of the residuals, i.e., the difference between input data and predicted values by the RS model. Graphical analysis of residuals includes plotting the residuals against time or run order, comparison of the residual versus fitted values and normal probability plots. 


\section{Experimental case studies}

This chapter describes the experimental facilities, measurement equipment and geometrical properties of the two confluent jet setups that have been investigated experimentally. The chapter also provides information on measurement procedure and measurement locations.

All measurements carried out in the present work were conducted at the Department of Process and Energy, Faculty Mechanical, Maritime and Materials Engineering, Delft University of Technology, The Netherlands. The experimental cases studies include LDA and PIV measurements of two confluent jet array configurations; one $6 \times 6$ jet array and one $1 \times 6$ jet array. Further details on experimental setups and measurement procedure are found in Paper I and II.

\subsection{Test facilities and seeding generation}

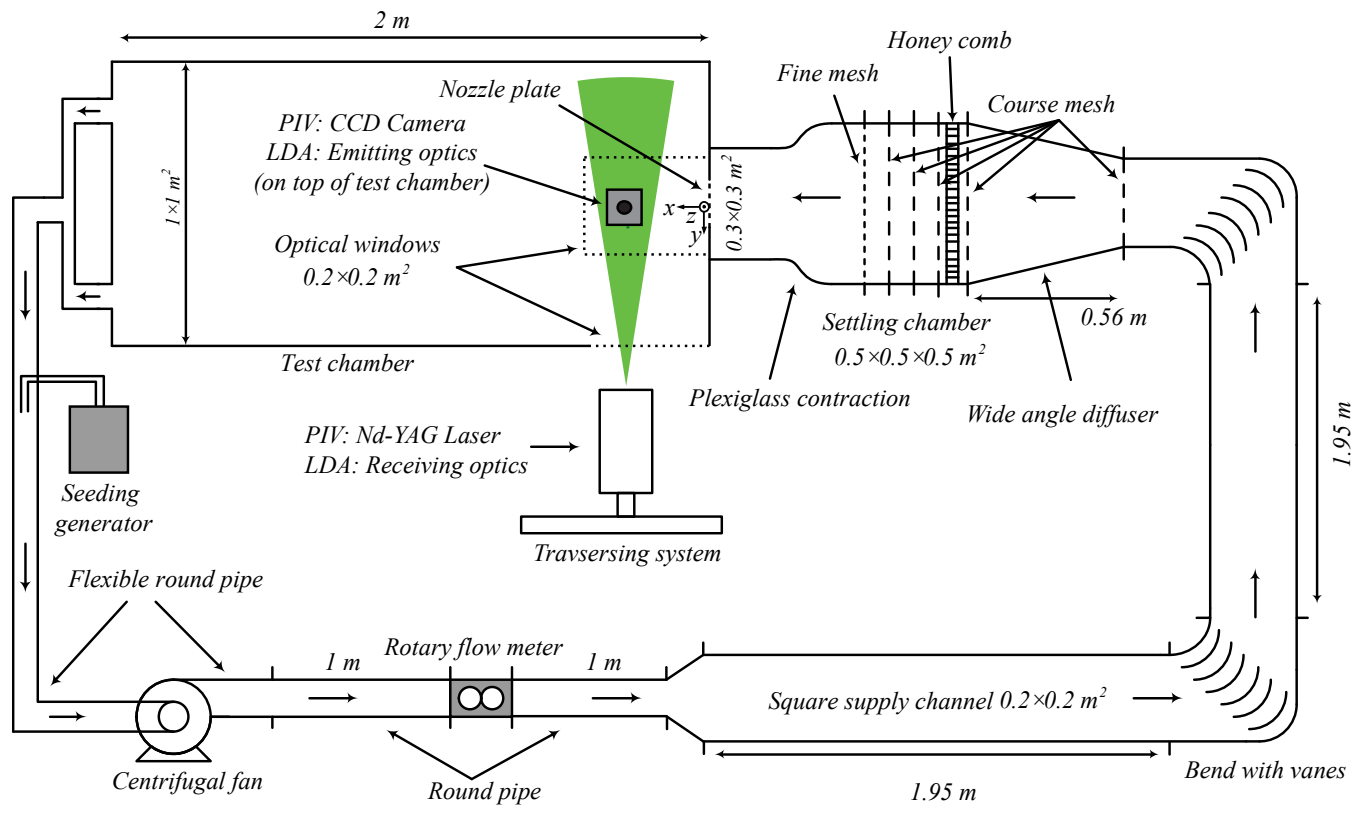

Figure 15 Top-view schematic illustration of wind tunnel and test chamber.

In Figure 15 a schematic illustration of the wind tunnel, designed to create a uniform low-turbulence flow upstream of the nozzle plate, is shown. The wind tunnel includes a controllable centrifugal fan that accelerates the air and increases air pressure. The air 
passes through a rotary flow meter, and then flows towards the test chamber through a supply channel system, diffusor, setting chamber and finally a square contraction.

The nozzle plates were mounted on the outlet of the contraction. The jets issue into a test chamber with dimensions $1 \times 1 \times 2 \mathrm{~m}$, which was built to avoid disturbances from room air movement. The test chamber was equipped with two high transmittance optical windows that enabled optical access during measurements. Details of the wind tunnel setup and information on the wind tunnel equipment are found in Table 6. Further information on the settling chamber and supply ducts of the wind tunnel can be found in Geers (2004).

The seeding system was the same during both PIV and LDA measurements. Seeding was generated with Laskin nozzle, submerged in an aqueous di-alcohol glycerol mixture. The seeding particles had a mean diameter of approximately $1 \mu \mathrm{m}$ and were injected upstream of the ventilator and recycled in the closed loop of the wind tunnel. This configuration ensured a homogeneous seeding concentration in the jets and the ambient.

Table 6 Wind tunnel properties.

\begin{tabular}{|c|c|c|}
\hline Fan & & ITHO, MD-63 \\
\hline \multicolumn{3}{|c|}{ Flow meter } \\
\hline & $6 \times 6$ jet setup & Instromet, Silvolde NL, Q-75-E \\
\hline & $1 \times 6$ jet setup & Elster-Instromet, IRM-1 DN50 G25 \\
\hline \multicolumn{3}{|c|}{ Wind tunnel flow rate $\left(Q_{0}\right)$} \\
\hline & $6 \times 6$ jet setup & $5.3 \times 10^{-3}$ to $11.4 \times 10^{-3} \mathrm{~m}^{3} / \mathrm{s}$ \\
\hline & $1 \times 6$ jet setup & $0.9 \times 10^{-3}$ to $1.9 \times 10^{-3} \mathrm{~m}^{3} / \mathrm{s}$ \\
\hline Inlet & locity of jets, $U_{b}=Q_{0} / A_{\text {inlet }}$ & 5.6 to $12 \mathrm{~m} / \mathrm{s}$ \\
\hline \multicolumn{3}{|c|}{ Diffusor } \\
\hline & Inlet area & $200 \times 200 \mathrm{~mm}^{2}$ \\
\hline & Outlet area & $500 \times 500 \mathrm{~mm}^{2}$ \\
\hline & Length & $560 \mathrm{~mm}$ \\
\hline \multicolumn{3}{|c|}{ Settling chamber } \\
\hline & Dimensions & $500 \times 500 \times 500 \mathrm{~mm}^{2}$ \\
\hline & Honeycomb & cell diameter $3.4 \mathrm{~mm}$, length $80 \mathrm{~mm}$ \\
\hline & Coarse mesh & open area ratio 0.59 , wire diameter $1 \mathrm{~mm}$ \\
\hline & Fine mesh & open area ratio 0.69 , wire diameter $0.25 \mathrm{~mm}$ \\
\hline \multicolumn{3}{|c|}{ Contraction } \\
\hline & Inlet area & $500 \times 500 \mathrm{~mm}^{2}$ \\
\hline & Outlet area & $300 \times 300 \mathrm{~mm}^{2}$ \\
\hline & Length & $620 \mathrm{~mm}$ \\
\hline
\end{tabular}

\subsection{Nozzle plate design and jet categories}

The nozzles were arranged as either a $6 \times 6$ square in-line array of jets or a single row of $1 \times 6$ jets. In both setups the nozzle diameter $\left(d_{0}\right)$ was $5.8 \mathrm{~mm}$. The nozzle spacing $(S)$ was $2.82 d_{0}$. The nozzles were of a short converging type with a height $(h)$ of $0.4 d_{0}$. 
The inlet bulk velocity, calculated according to Eq. (73) was altered between 5.6 and $12 \mathrm{~m} / \mathrm{s}$, resulting in Reynolds numbers based on the inlet diameter, $R e_{d}$, between 2125 and 4555.

$U_{b}=Q_{0} / A_{\text {inlet }}$

where $Q_{0}$ is the inlet flow rate, measured by the flow meter and $A_{\text {inlet }}$ is the total area of the jet inlets, i.e., $36 \pi / 4 \cdot d_{0}^{2}$ and $6 \pi / 4 \cdot d_{0}^{2}$ in the $6 \times 6$ and $1 \times 6$ setup respectively.

In both setups the origin of the Cartesian coordinate system was set to the center of the nozzle located directly on the right-hand side of the vertical symmetry plane, and in the case of the $6 \times 6$ jet array, also located directly above the horizontal symmetry plane. The $x$-coordinate is perpendicular to the nozzle plate, the $y$-and $z$-coordinate is oriented horizontally and vertically. The nozzle shape and location of the coordinate system for the two setups is presented in Figure 16.
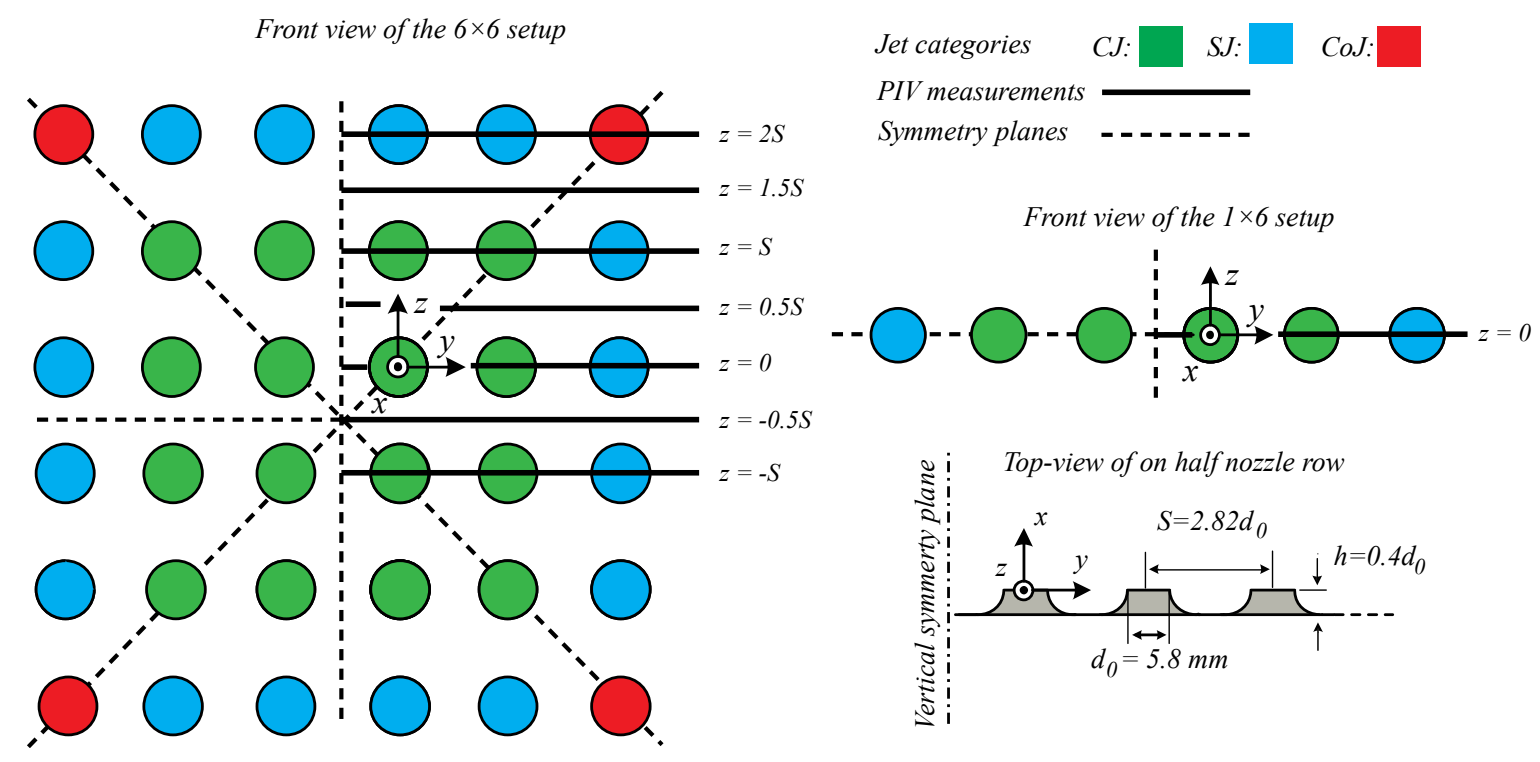

Figure 16 Coordinate system, geometrical details, location of PIV measurements and categorization of jets, based to the number of surrounding jets, in the two experimental case studies.

Throughout the present work the jets within the arrays are divided in categories based on the number of surrounding jets. This facilitates the presentation of results, in terms of location. Jets in different categories also differ in behavior and therefore the definition also has physical meaning (see for example Paper I and IV).

In a square in-line array, such as the $6 \times 6$ array in the experimental case study, the jet categories are called Central Jets (CJ), located in the center of the array; Side Jets (SJ), located along the sides of the array; and Corner Jets (CoJ), located at the corners of the array. In square or rectangular arrays the three categories of jets have eight, five and three neighboring jets, respectively. The locations of the three different jet categories are visualized in Figure 16. In a single row of jets the two jets at the edges of the row are denoted as Side Jets (SJ), while other jets are called Central Jets (CJ). In a single row of jets $S J s$ and $C J$ s thereby have one and two neighboring jets respectively (see Figure 16). It is worth mentioning that there is more than one unique position of $C J \mathrm{~s}$ and 
SJs in square arrays and CJs in the single row of jets. This means that not all jets within these categories are expected to behave identically.

\subsection{PIV measurements}

A double pulsed Nd:YAG laser (Continuum Minilight), with a pulse energy of $25 \mathrm{~mJ}$ was used to illuminate the seeding particles. The light sheet optics produced a laser sheet thickness of approximately $1 \mathrm{~mm}$. Scattered light from the particles was recorded on a $1280 \times 1024$ pixel CCD sensor (PCO SensiCam). CamWare 3.08 and an ILA timing unit were used to acquire images and synchronize the laser shoots and the camera. All PIV measurements were carried out at three different Reynolds numbers. Table 7 presents a summary of recording parameters during PIV measurements.

Table 7 PIV recording parameters for all measurements.

\begin{tabular}{|c|c|c|c|}
\hline Focal length of lens (mm) & \multicolumn{3}{|c|}{105} \\
\hline Relative aperture & \multicolumn{3}{|c|}{11} \\
\hline Field of view $\left(\mathrm{mm}^{2}\right)$ & \multicolumn{3}{|c|}{$48 \times 38$} \\
\hline Final interrogation area (pixels) & \multicolumn{3}{|c|}{$16 \times 16$} \\
\hline Spatial resolution (mm/pixel) & \multicolumn{3}{|c|}{0.037} \\
\hline Interrogation overlap $(\%)$ & \multicolumn{3}{|c|}{$\begin{array}{l}6 \times 6 \text { setup: } 50 \\
1 \times 6 \text { setup: } 25\end{array}$} \\
\hline Reynolds number, $R e_{d}$ & 2125 & 3290 & 4555 \\
\hline Pulse delay $(\mu s)$ & 39 & 25 & 18 \\
\hline Inlet bulk velocity, $U_{b}(\mathrm{~m} / \mathrm{s})$ & 5.6 & 8.6 & 12 \\
\hline Number of snapshots & \multicolumn{3}{|c|}{4000} \\
\hline Time lag between image pairs (s) & \multicolumn{3}{|c|}{0.5} \\
\hline
\end{tabular}

The commercial software DaVis v8.08 was used for analyzing the image pairs and computing the vector field. After an image preprocessing with particle intensity normalization the image pairs were processed in two consecutive multi-pass steps with decreasing interrogation area size. The first step used square interrogation areas with a window size of $32 \times 32$ pixels. The resulting particle displacements were used as window displacements for a cross correlation with interrogation area of $16 \times 16$ pixels and either $25 \%$ or $50 \%$ window overlap. A local median filter was used to remove spurious vectors and the resulting empty spaces were filled up by interpolation. Following the practical guidelines by Adrian and Westerweel (2010), the uncertainty of the instantaneous velocity field was estimated to between $3 \%$ and $5 \%$ of the bulk velocity. The maximum statistical error due to the finite number of samples was less than $\pm 0.014 U_{b}$ for mean velocities and less than $\pm 0.008 U_{b}^{2}$ and $\pm 0.003 U_{b}^{2}$ for normal and shear Reynolds stresses. For the Reynolds stresses this represents about 6\% of the maximum values of the stresses in the shear layers. 


\subsection{LDA measurements}

The stream-wise velocity component was measured with the green channel (514.5 $\mathrm{nm}$ ) of a $4 \mathrm{~W}$ argon-ion laser (Spectra Physics, model 2016). One of the laser beams was frequency pre-shifted $40 \mathrm{MHz}$ by a Bragg cell to enable the detection of flow direction. The scattered light was collected in side scatter and the effective length and diameter of the measurement volume was approximately 0.15 and $0.1 \mathrm{~mm}$, respectively. The output signal of the photomultiplier was electronically down-mixed to an effective pre-shift frequency of $5 \mathrm{MHz}$ and fed to a Dantec BSA signal processor. About 6,000 validated velocity samples were taken at each measurement location. Velocity statistics were computed using transit time weighing to avoid errors due to velocity bias. The overall uncertainty of the LDA measurements is about $2 \%$ of the bulk velocity for the mean velocity and $8 \%$ of the local maximum for the Reynolds stress.

\subsection{Measurement locations}

All PIV measurements were performed in planes perpendicular to the nozzle plate $(z=$ constant). The PIV measurements thereby include the stream-wise velocity component in the $x$-direction $\left(U, u^{\prime}\right)$ and the span-wise velocity component in the horizontal $(y)$ direction $\left(V, v^{\prime}\right)$. The span-wise velocity in the vertical $(z)$ direction $\left(W, w^{\prime}\right)$ is the out-of-plane component and is not included in the planar PIV measurements.

In the $1 \times 6$ array PIV measurements were conducted at the center plane of the nozzles $(z=0)$, while in the $6 \times 6$ array PIV measurements were conducted in seven different planes in the array. The locations of PIV measurements are presented in Figure 16. The measurements were limited to the right-hand side of the vertical symmetry plane and for the $6 \times 6$ array mainly conducted in the upper right quadrant of the array. The PIV measurements thereby cover three out of six jets in each row. This increases the spatial resolution compared to measuring across the full width of the jet array. The symmetry of the $6 \times 6$ array was verified by measurements on both sides of the horizontal symmetry plane.

Each measurement plane consists of between four and six PIV recording sessions, together covering the area from the nozzle exit up to a maximum downstream distance between $20.6 \leq x / d_{0} \leq 29$. The field of view in adjacent recording sessions slightly overlapped so that regions with high in-plane loss of pairs, close to the edge of the field of view, can be cut out without creating gaps in the velocity fields. The LDA measurements were mainly conducted in the plane $z=0$ and for $R e_{d}=3290$. In Figure 17 the layout of PIV recording sessions and locations of LDA measurements in the plane $z=0$ are presented for both the $1 \times 6$ and the $6 \times 6$ setup. 


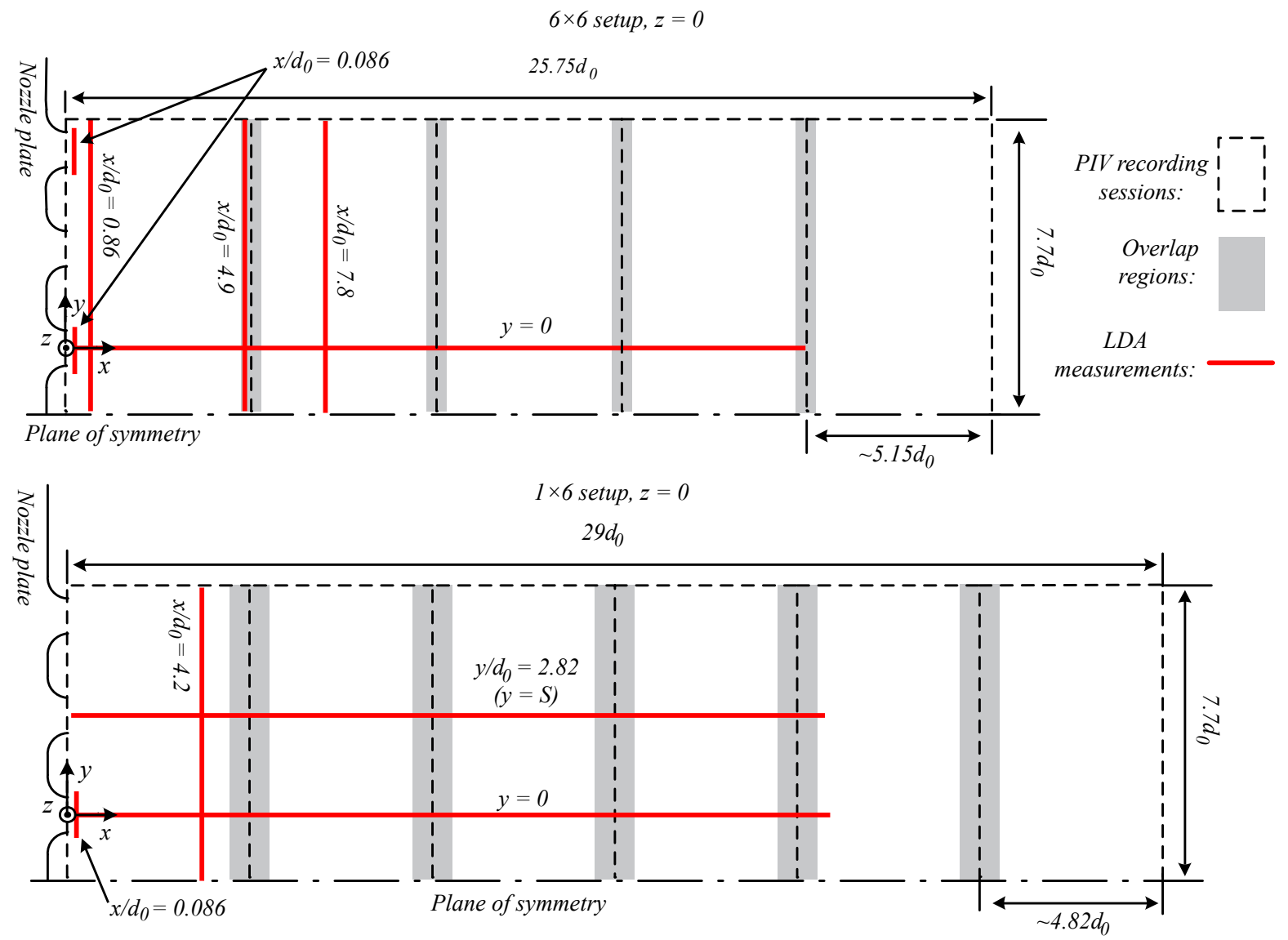

Figure 17 Location of PIV and LDA measurements in the plane $z=0$ for $6 \times 6$ (top) and $1 \times 6$ setup (bottom). 


\section{Computational model}

In this chapter the CFD models used in the present work are presented. The chapter provides information on mesh generation, boundary conditions and solution strategy. The procedures by which inlet boundary conditions are generated from experimental data in the computational study (Paper III and IV) are explained. Finally the setup of the parametrical study in Paper $V$ and VI is presented.

In Paper III and IV a CFD model with the same geometry as the $6 \times 6$ jet array setup in the experimental study was constructed and validated towards experimental PIV data. In Paper V and VI the validated CFD model was used for parametrical investigation. In this chapter the CFD models in the present work are described. The governing equations and employed turbulence models were presented in Chapter 4. Results from the CFD simulations and comparison with experimental data are found in Chapter 8.

\subsection{Case study: $6 \times 6$ confluent jet array}

This section presents the CFD model in Paper III and IV, designed to represent a computational model of the experimental case study of the $6 \times 6$ confluent jet array in Paper I and II. Geometrical details of the investigated array are found in Section 6.2.

\subsubsection{Computational model and numerical details}

The CFD model has a rectangular shape, with dimensions $H \times W \times L$. Figure 18 shows a sketch of the computational domain and implemented boundary conditions. Due to the symmetrical appearance of the confluent jet array, only the nine jets located in the upper right quadrant of the array, where the majority of the PIV measurements were performed, are included in the CFD model. The diagonal line $y=z$ also forms a plane of symmetry. Using this symmetry condition reduced the computational effort, however also resulting in a mesh which had cells with higher skewness. This had a negative influence on convergence and therefore it was found more effective not to use the possibility to model only $1 / 8$ of the nozzle plate. The CFD simulations of the $6 \times 6$ array were conducted at the intermediate Reynolds number $\left(R e_{d}=3290\right)$, out of the three Reynolds numbers investigated experimentally. This corresponds to an inlet bulk velocity, $U_{b}$, of approximately $8.6 \mathrm{~m} / \mathrm{s}$.

Table 8 presents a summary of parameters of the CFD models in Paper III and IV. All simulations were carried out with the commercial CFD solver ANSYS Fluent 
13.0. The non-linear convective terms were discretized with the second-order upwind scheme, while second-order central differencing was used for the viscous terms. The SIMPLE algorithm was used to handle the pressure-velocity coupling. The no-slip condition and either standard or scalable wall functions were applied for the wall and symmetric boundary conditions were used for the symmetry planes. In the far extent of the computational domain a constant static pressure of $0 \mathrm{~Pa}$, relative to the CFD model operating pressure of $101.3 \mathrm{kPa}$ was applied as boundary condition. For the far field boundaries parallel to the flow direction the boundary conditions were either symmetry planes or a constant static pressure. The CFD model was considered converged when residuals of all variables were less than $10^{-5}$ and the solution had stabilized.
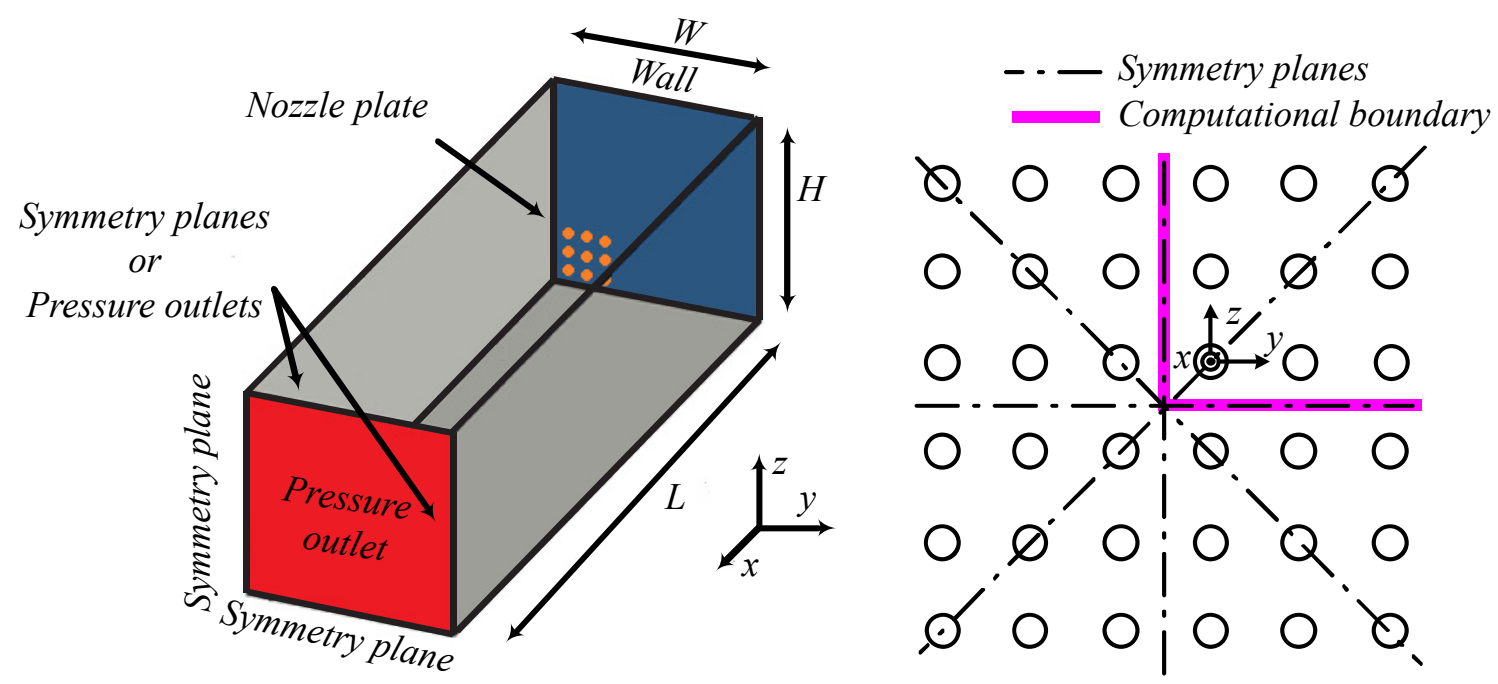

Figure 18 Overview of computational model (left). Use of nozzle plate symmetry in computational model (right).

Table 8 CFD model properties for the $6 \times 6$ jet array case studies in Paper III and IV.

\begin{tabular}{|c|c|c|}
\hline Properties & Paper III & Paper IV \\
\hline CFD solver & $\begin{array}{c}\text { Fluent } \begin{array}{c}13.0, \text { segregated } \\
\text { solver }\end{array}\end{array}$ & $\begin{array}{c}\text { Fluent } \begin{array}{c}13.0, \text { segregated } \\
\text { solver }\end{array}\end{array}$ \\
\hline Turbulence model & RSM & $k-\varepsilon$ std., RNG k-e, RSM \\
\hline $\begin{array}{c}\text { Discretization scheme, } \\
\text { convective terms }\end{array}$ & $2^{\text {nd }}$ order upwind & $2^{\text {nd }}$ order upwind \\
\hline Domain size $H \times W \times L[\mathrm{~mm}]$ & $423.5 \times 423.5 \times 1425$ & $423.5 \times 423.5 \times 1425$ \\
\hline Grid size & $3,027,480$ & $4,374,000$ \\
\hline Inlet profiles & $U, V, W, \overline{u^{\prime 2}}, k, \varepsilon$ & $U, V, W, \overline{u^{\prime 2}}, \overline{v^{\prime 2}}, \overline{w^{\prime 2}}, k, \varepsilon$ \\
\hline Wall treatment & Standard wall functions & Scalable wall functions \\
\hline $\begin{array}{c}\text { Far field boundary } \\
\text { condition, } \\
\text { parallel to flow direction }\end{array}$ & Constant pressure $(0 \mathrm{~Pa})$ & $\begin{array}{l}\text { Symmetry conditions } \\
\text { (slip wall) }\end{array}$ \\
\hline
\end{tabular}




\subsubsection{Generation of inlet boundary conditions}

The geometry of the nozzle plate was modified in the CFD model, where the nozzles were extended and widened as laser sheet reflections from the nozzle plate made it difficult to acquire reliable PIV data for inlet boundary conditions upstream of $x / d_{0}=0.86$. A comparison between the nozzle plate geometry in the experimental setup and the geometry used in the CFD model can be found in Figure 19.

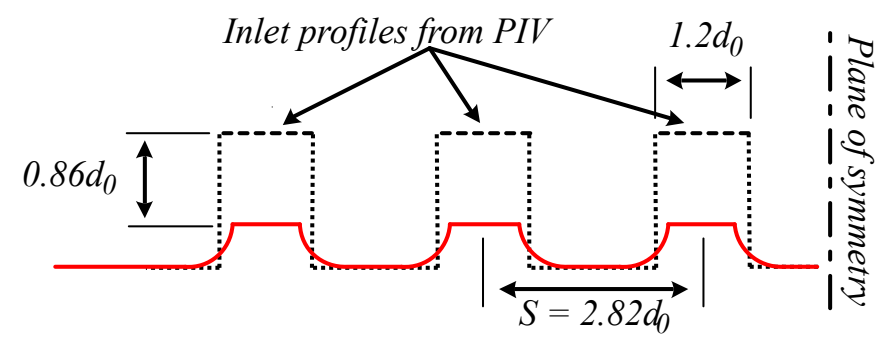

Figure 19 Nozzle shape in experimental setup (solid lines), location of PIV inlet boundary data (dashed lines) and nozzle geometry used in CFD model (dotted lines).

As the PIV measurements are performed at the horizontal center planes of the jets, approximations are needed to generate complete inlet profiles based on experimental data for the CFD model. The methods by which inlet boundary conditions are generated are explained below.

In Paper III the inlet boundary conditions were based on experimental data for three out of the nine jets included in the CFD model. Inlet profiles of $U, V$ and $\overline{u^{\prime 2}}$ were generated from measurements of the jets located at $z=0$ (see coordinate system in Figure 18). Inlet boundary conditions for the other jets were generated based on the assumption that the profiles of $U, V$ and $\overline{u^{\prime 2}}$ depend on the distance from the jet axis to the vertical plane of symmetry. $W$ was attained by using the symmetry between the horizontal $(y)$ and vertical $(z)$ direction. $\overline{u^{\prime} v^{\prime}}$ was found to be negligible compared to normal stresses and since the other two shear stresses were not measured, all shear stresses were set to zero. $\overline{v^{\prime 2}}$ and $\overline{w^{\prime 2}}$ were also set to zero and $k$ approximated as $k=1 / 2 \overline{u^{\prime 2}}$.

In Paper IV the procedure of generating inlet conditions was refined and used PIV data from all jets included in the CFD model. PIV measurements at $z=0, S$ and $2 S$ (nozzle horizontal center planes) were used for inlet profiles of $U, V, \overline{u^{\prime 2}}$ and $\overline{v^{\prime 2}}$. Values of $U, W, \overline{u^{\prime 2}}$ and $\overline{w^{\prime 2}}$ were then approximated along the nozzles' vertical center planes using the diagonal $(y=z)$ symmetry of the setup. Figure 20 shows how PIV data for the jets at $\left[y_{c}, z_{c}\right]=[0,0]$ and $\left[y_{c}, z_{c}\right]=[2 S, 0]$ are used as inlet boundary data for other jets in the CFD model in Paper III and IV. $y_{c}$ and $z_{c}$ are the coordinates of the jet geometrical axis. 


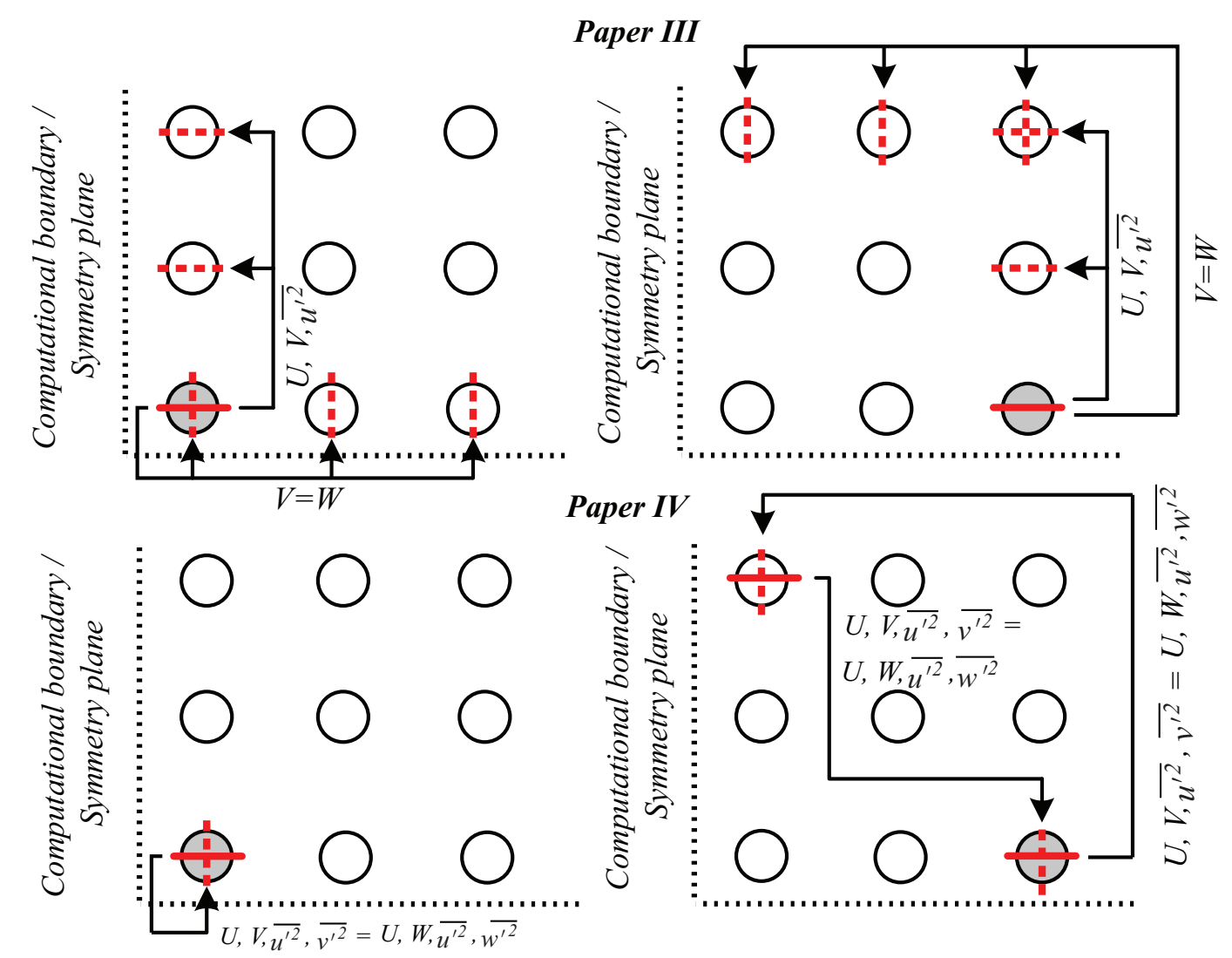

Figure 20 Methods for generating inlet boundary conditions for the jets at $\left[y_{c}, z_{c}\right]=[0,0]$ (left) and $\left[y_{c}, z_{c}\right]=[2 S, 0]$ (right) in Paper III (top) and Paper IV (bottom).

From the PIV data, piecewise spline fits are generated, which are then rotated around the jet geometrical axis, to generate complete inlet profiles for the CFD model (see Paper IV for further information). Figure 21 shows the resulting complete mean velocity inlet profiles for the jet located at $\left[y_{c}, z_{c}\right]=[2 S, 0]$ in Paper III and IV. The coordinates $\left[y^{\prime}, z^{\prime}\right]$ are local coordinates for the investigated jet, defined as $\left[y^{\prime}, z^{\prime}\right]=[y, z]-\left[y_{c}, z_{c}\right]$. This jet is located along the side of the array, as will be discussed in Chapter 8, SJs are both deformed and displaced towards the array center. The profile of stream-wise velocity, $U$, has a top-hat similar appearance and is somewhat flattened in the horizontal $\left(y^{\prime}\right)$ direction due to the previously mentioned jet deformation. The inlet profile of $V$ is the same in Paper III and IV for the jet under consideration and the negative values reflect the inwards displacement of the jet. In Paper III the data for $W$ is extracted from the jet at the array center (see Figure 20), which has very low span-wise movement. In Paper IV, the data of $W$ comes from the mirrored jet on the other side of the diagonal symmetry plane. The inlet profile of $W$ in Paper IV will tend to stretch the jet in the vertical direction. As will be shown in Section 8.4.1, the boundary conditions in Paper IV, which better account for the deformation process already at the CFD model inlet, also better reproduce experimental results further downstream. 

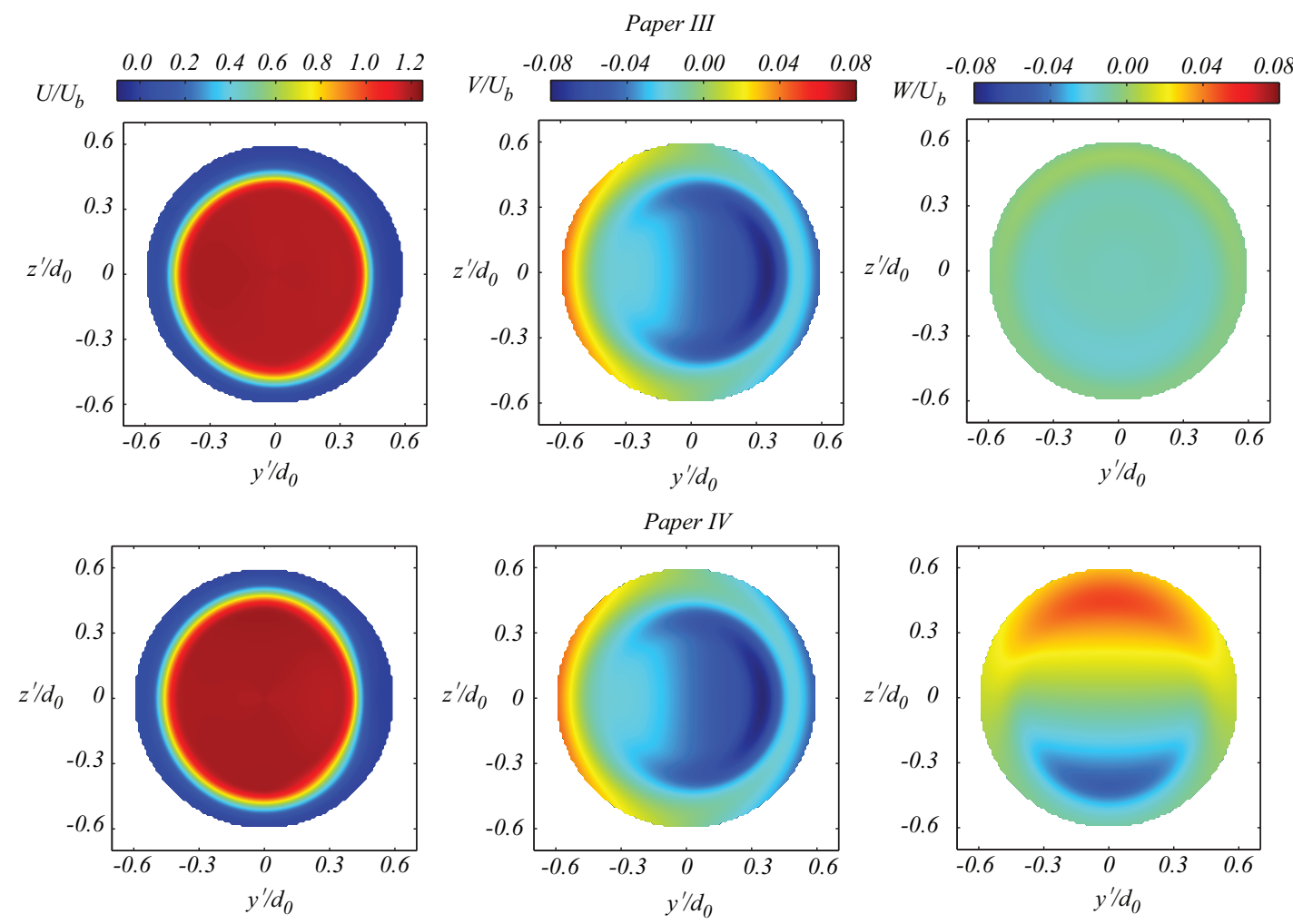

Figure 21 Inlet boundary conditions of mean velocity components for the jet located at $\left[y_{c}, z_{c}\right]=[25,0]$ in Paper III (top) and IV (bottom).

\subsubsection{Mesh strategy and grid independence}

In all CFD simulations the mesh consists of structured hexahedral cells. Due to the occurrence of high velocity gradients the grid is refined close to the nozzles (see Figure 22). The grid independency was tested using different mesh densities and grid configurations. Results of grid independency tests are presented in Paper IV and V.
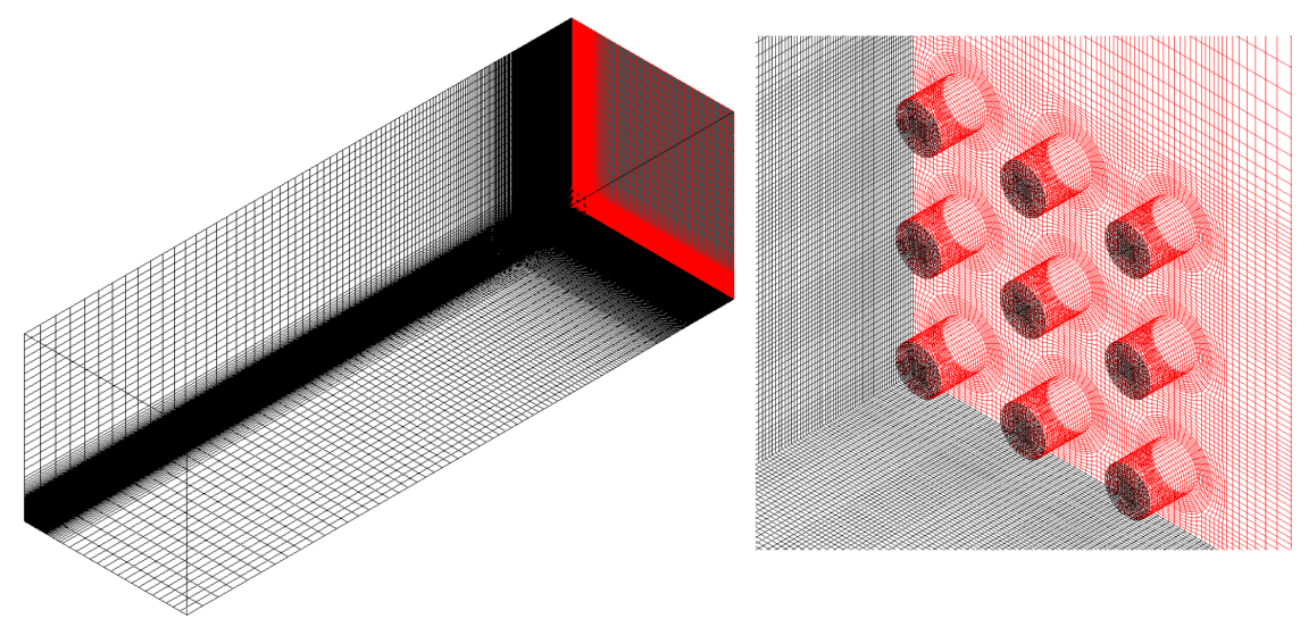

Figure 22 Overview of the computational grid with 4,374,000 cells (left) and zoom in of nozzles (right) used in Paper IV. 
In Figure 23 the development of inverse maximum stream-wise velocity in the jet array, $U_{b} / U_{\max }$, is shown for three different grid densities. The effect of grid density is small and it was concluded that a grid density corresponding to 4,374,000 cells was considered sufficient for the case study in Paper IV.

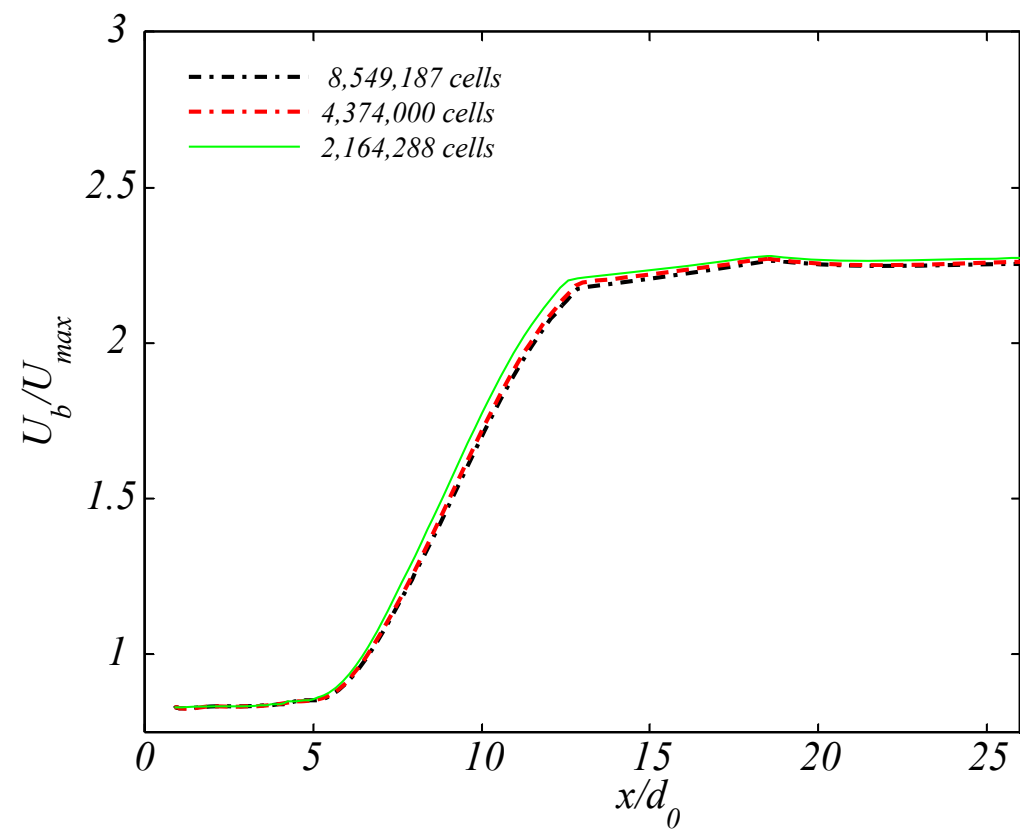

Figure $23 U_{b} / U_{\max }$ in the $6 \times 6$ jet array for different grid resolutions (RSM turbulence model).

\subsection{Parametrical investigation: CFD models and statistical approximation}

The parametrical investigation in Paper $\mathrm{V}$ uses statistical methods in the form of Response Surface Methodology and Design of Experiments to fit a second-order polynomial response surface (RS) to a set of design points, where data of investigated response variables has been acquired through CFD simulations. The RS models were based on a three-level fractional factorial design using Box-Behnken design method and included five independent factors (parameters), which resulted in a total number of 41 design points (runs). In Table 9 investigated factors and their level values within the parametrical investigation are presented. Figure 24 show an example of the geometry close to the nozzle inlet for the CFD model used in the parametrical investigation. The figure illustrates the meaning of the factors $n, h, d_{0}$ and $E$ (see Table 9). The response variables investigated in Paper $\mathrm{V}$ are summarized in Table 11, Section 8.6. Descriptions of the employed statistical methods are found in Chapter 5. 
Table 9 Values of independent factors for the design space in Paper V

\begin{tabular}{cccc}
\hline Factor & Low level & Medium level & High level \\
\hline Number of jets per row, $n[-]$ & 6 & 8 & 10 \\
Nozzle edge-to-edge spacing, $E[\mathrm{~mm}]$ & 6 & 9 & 12 \\
Inlet nozzle diameter, $d_{0}[\mathrm{~mm}]$ & 4 & 8 & 12 \\
Inlet velocity, $U_{b}[\mathrm{~m} / \mathrm{s}]$ & 4 & 8 & 12 \\
Nozzle height, $h[\mathrm{~mm}]$ & 4 & 8 & 12 \\
\hline
\end{tabular}

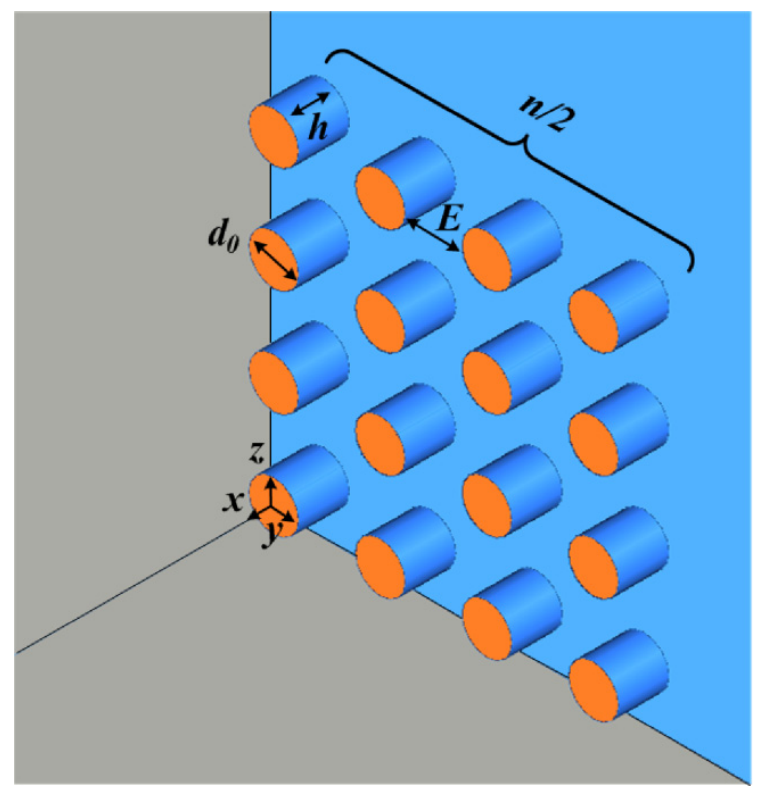

Figure 24 Example of the nozzle plate geometry in CFD model used for parametrical investigation.

In Paper VI further investigation on 4 out of 41 CFD simulation setups (design points) in Paper $\mathrm{V}$ was conducted, discussing the influence of dimensionless jet spacing and confluent jet array size on jet interactions and development of combined jet. The main objective of the RS models in Paper V is identifying principal influence of included factors on the response variables rather than achieving an exact prediction of a certain setup, as in Paper III and IV. Therefore inlet boundary conditions in the CFD models used in Paper V and VI were simplified, using uniform profiles of inlet velocity and turbulence intensity. However, as will be shown in Section 8.6.1 the resulting RS models are capable of predicting the response variable values in the validation setup in Paper IV.

The CFD simulations in Paper V and VI were performed in ANSYS Fluent 14.5 and used the $k-\varepsilon s t d$. turbulence model due to better convergence compared to RSM, and the large number of simulations to be conducted. Other aspects such as wall and farfield boundary conditions, discretization and convergence criteria were the same in Paper V and VI as in the validation case study in Paper IV. 
The mesh resolution was similar to Paper IV in all runs and a grid independency test was performed for the center-point run in Paper V, i.e., when all factors are held at the middle level. However as the number of jets is larger in some of the runs the mesh size varied between approximately 4.5 and 23.9 million cells in Paper V. Special care was also taken to ensure that the far-field boundaries did not influence the solution when the number of jets increased. A larger computational domain was therefore used is some of the CFD simulations in Paper V and VI. 


\section{Results}

In this chapter the results from the experimental, computational and parametrical investigations are presented. The main purpose of the chapter is to summarize the results of the present work in relation to the imposed research questions.

However, aspects that are important to the present work but do not directly address the research questions are also discussed. This includes investigation of inlet profiles for different nozzles, comparison of experimental results acquired with different measurement techniques (LDA and PIV), the impact of assumptions regarding inlet boundary conditions in the CFD models and the possibility to describe the flow behavior based on derived response surface models.

The result chapter is divided in six sections. At first (Section 8.1) LDA measurements of nozzle inlet profiles are reported on in order to verify that the inlet conditions of different jets are similar to each other. Section 8.2 provides a comparison between PIV and LDA measurements. Section 8.3 compares results of the experimental investigation on the $6 \times 6$ and $1 \times 6$ jet arrays described in Chapter 6 . The main purpose is to identify principal differences between the two setups, and thereby represents a case study comparing a square in-line array and a single row of jets. In Section 8.4 CFD results of the $6 \times 6$ array are compared with PIV data and the impact of inlet boundary conditions and turbulence model is discussed. Section 8.5 reports on both computational and experimental results of the $6 \times 6$ array and summarizes the flow field development in an unconfined (square) confluent jet array through the case study of the $6 \times 6$ array. In the last section (8.6) the results from the parametrical investigations are summarized. The accuracy of derived response surface models is also discussed.

\subsection{Inlet profiles}

LDA measurements of the stream-wise velocity component were performed directly downstream of four different nozzles. For the $6 \times 6$ array measurements were conducted for one jet in each jet category (CJ, SJ and CoJ). For the $1 \times 6$ array measurements were performed for one of the $C J$ s. In Figure 25 the resulting profiles of mean velocity and RMS, made dimensionless with the centerline velocity $U_{c l}$ are shown. As seen from the figure the inlet profiles of both $U / U_{c l}$ and $\sqrt{u^{\prime 2}} / U_{c l}$ are similar for all measured jets, which is a prerequisite for comparison between the setups and discussion on influence of jet position. The short converging nozzles cause vena contracta effects, reflected by the saddleback shape of $U / U_{c l}$, which has 
two off-center maxima of about $1.12-1.13$. In the core of the jets $\sqrt{u^{\prime 2}} / U_{c l}$ is uniform and approximately $2 \%$. The maximum turbulence intensity $\left(\sqrt{u^{\prime 2}} / U_{c l}\right)$ in the shear layers is 10 to $15 \%$. The variation in peak values is most likely caused by the finite size of the LDA probe volume in relation to the thin shear layers.
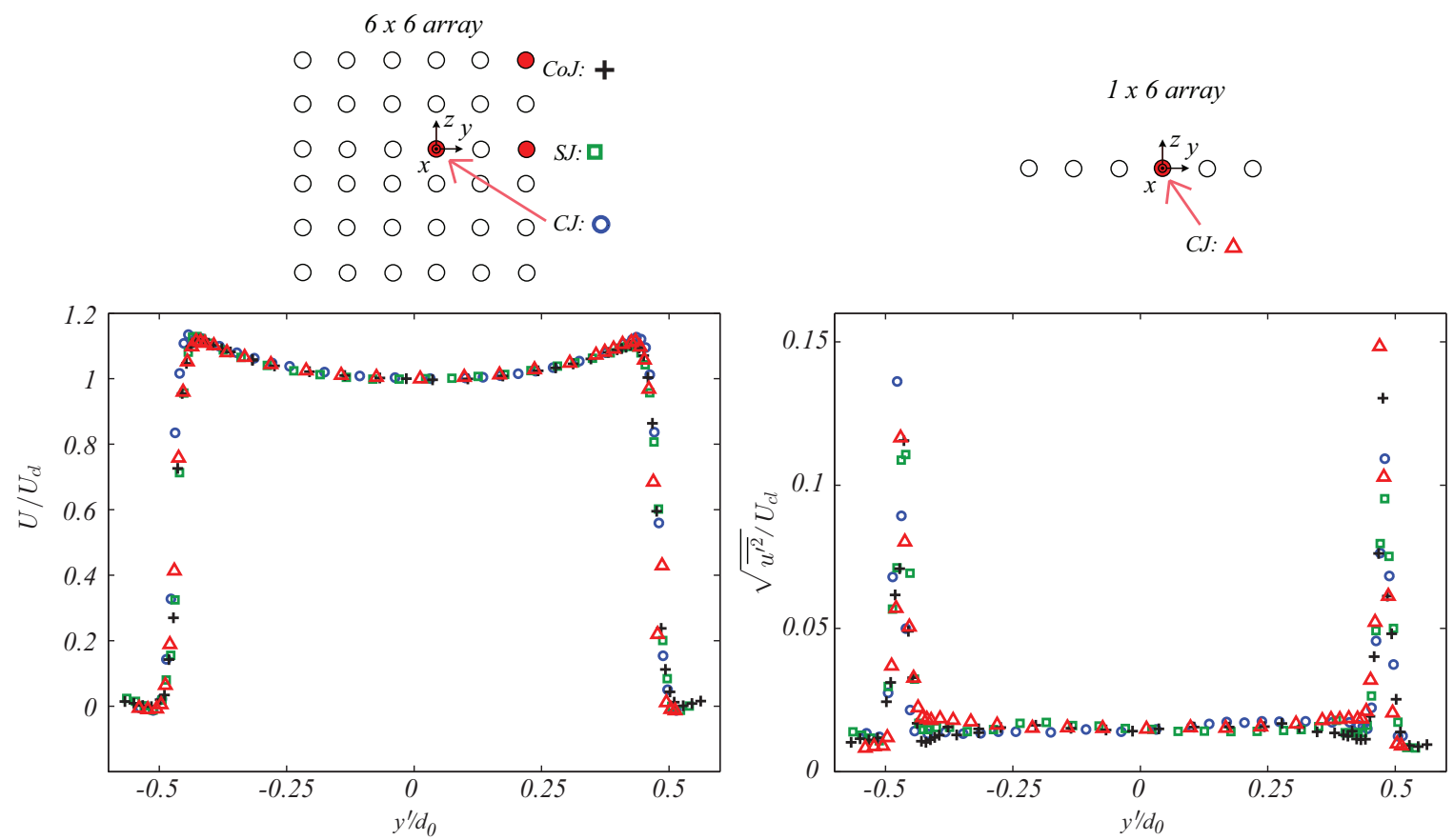

Figure 25 Measured profiles of dimensionless mean velocity in $x$-direction (left) and its RMS (right) at $x / d_{0}=0.086, R e_{d}=3,290$ (LDA)

\subsection{Comparison of measurement techniques}

This section presents a comparison of PIV and LDA measurements made in both the $6 \times 6$ and the $1 \times 6$ setup. The LDA measurements presented in this section are aimed at verifying the main findings of the PIV experiments. The results of PIV experiments are then used throughout the present work, both for pure experimental observations and validation of the CFD models.

In Figure 26 span-wise profiles of the stream-wise mean velocity $\left(U / U_{b}\right)$ and RMS, $\left(\sqrt{u^{\prime 2}} / U_{b}\right)$, located in the plane $z=0$ are presented for three locations $(x=$ constant $)$ in the $6 \times 6$ array and one location in the $1 \times 6$ array. There is overall good agreement between PIV and LDA results. Some differences can however be noted. In the $6 \times 6$ array the RMS show a difference in behavior around $y / d_{0}=4$ for the measurements at $x / d_{0}=4.9$. As will be shown later (see Section 8.6.2) this region, on the inside of the SJ, contains a rather complex flow, with vortices that cause out-of-plane motion, which might explain the differences between the measurement techniques. The $1 \times 6$ array also shows some difference in behavior on the inside of the $S J\left(y / d_{0} \approx 4\right)$, while the predictions elsewhere are in good agreement. Close to the nozzles $\left(x / d_{0}=0.86\right)$ the peak values of the RMS are associated with some uncertainties in the PIV measurements, due to the high velocity gradients in combination with the finite interrogation area size. 


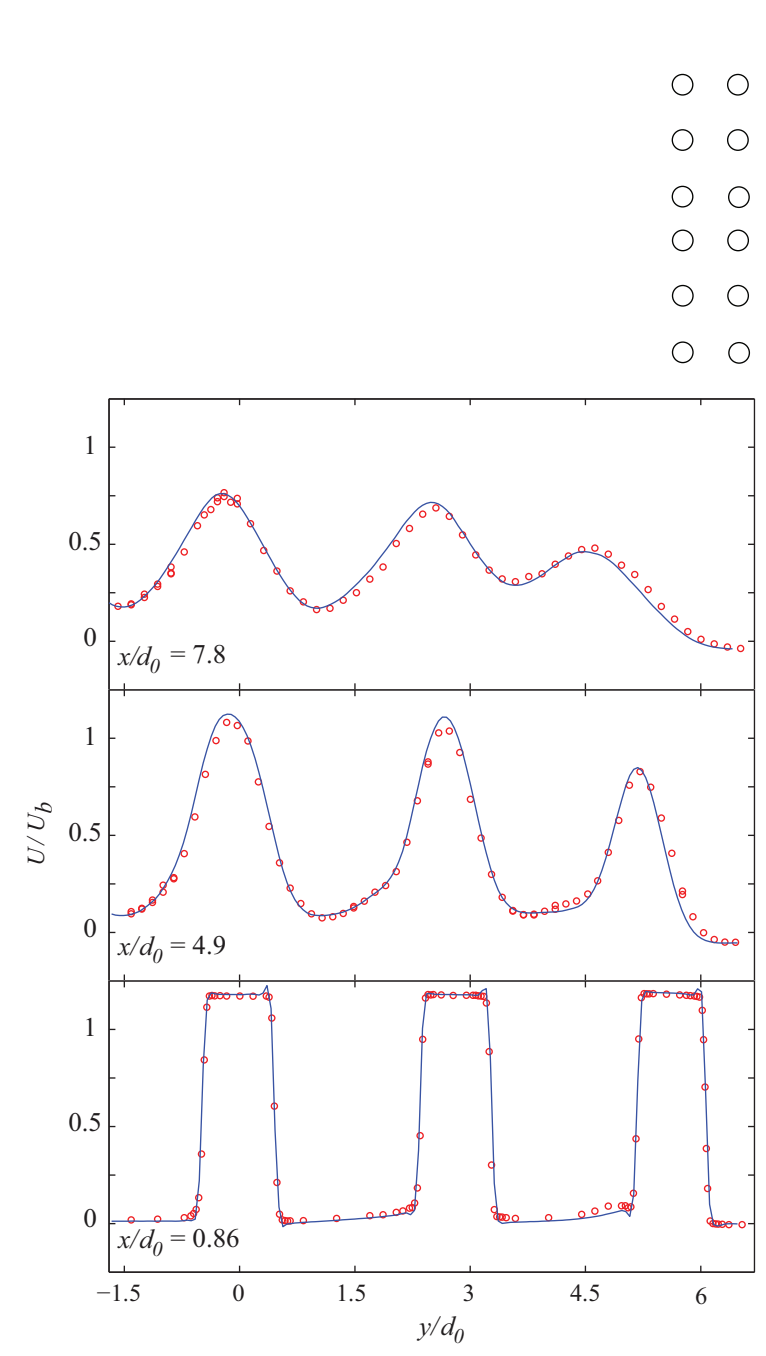

$6 \times 6$ array

$\circ \quad 0 \quad 0 \quad 0 \quad 0 \quad 0$

$0 \quad 0 \quad 0 \quad 0 \quad 0 \quad 0$

$\bigcirc \quad \bigcirc--\hat{A}^{z} y-\Theta--\Theta--$ PIV and LDA measurements, $z=0$

$\begin{array}{lllllllllll}0 & \circ & \circ & 0 & \circ & 0\end{array}$

$0 \quad 0 \quad 0 \quad 0 \quad 0$

$0 \quad 0 \quad 0 \quad 0 \quad 0 \quad 0$

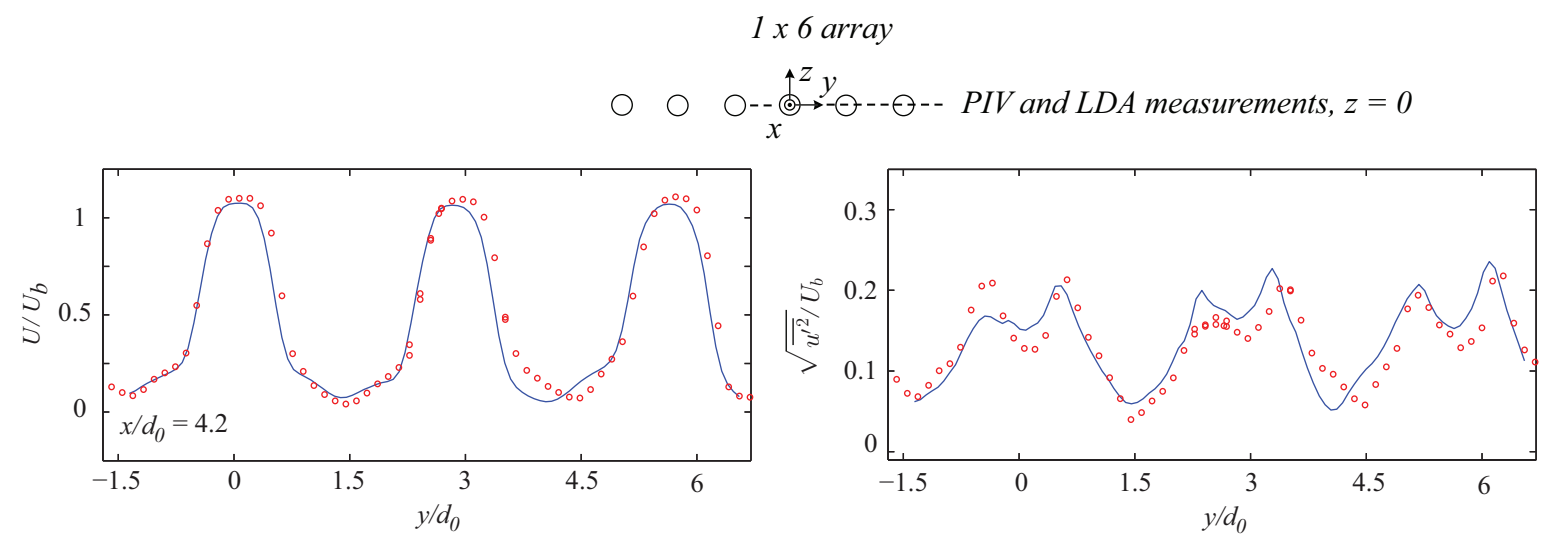

Figure 26 Span-wise profiles of dimensionless mean velocity in $x$-direction, $U / U_{b}$, (left) and the dimensionless RMS, $\sqrt{u^{\prime 2}} / U_{b}$ (right) measured by PIV (solid lines) and LDA (symbols) for $R e_{d}=3290$.

In Figure 27 measurement of $U / U_{b}$ and $\sqrt{\overline{u^{\prime 2}}} / U_{b}$ along the geometrical centerlines of one $C J$ in the $6 \times 6$ array and two $C J$ s in the $1 \times 6$ array are shown. Differences between LDA and PIV results are mainly found in regions associated with relatively high velocity gradients and might reflect the larger measurement volume, i.e., interrogation area and laser sheet thickness of the PIV measurements. However, the overall behavior is consistent between the two measurement techniques. 
It can be noted that the PIV results in Figure 27 span several separate PIV recordings. Some of the bumps seen in the PIV results in Figure 27 are related to the intersections between recording sessions. The recording sessions were made with some degree of overlap and in Paper II the conformity between adjacent recording sessions was investigated with satisfactory results. For the $6 \times 6$ array LDA measurements along the geometrical centerline were also performed for two other Reynolds numbers with similar results as presented in Figure 27.
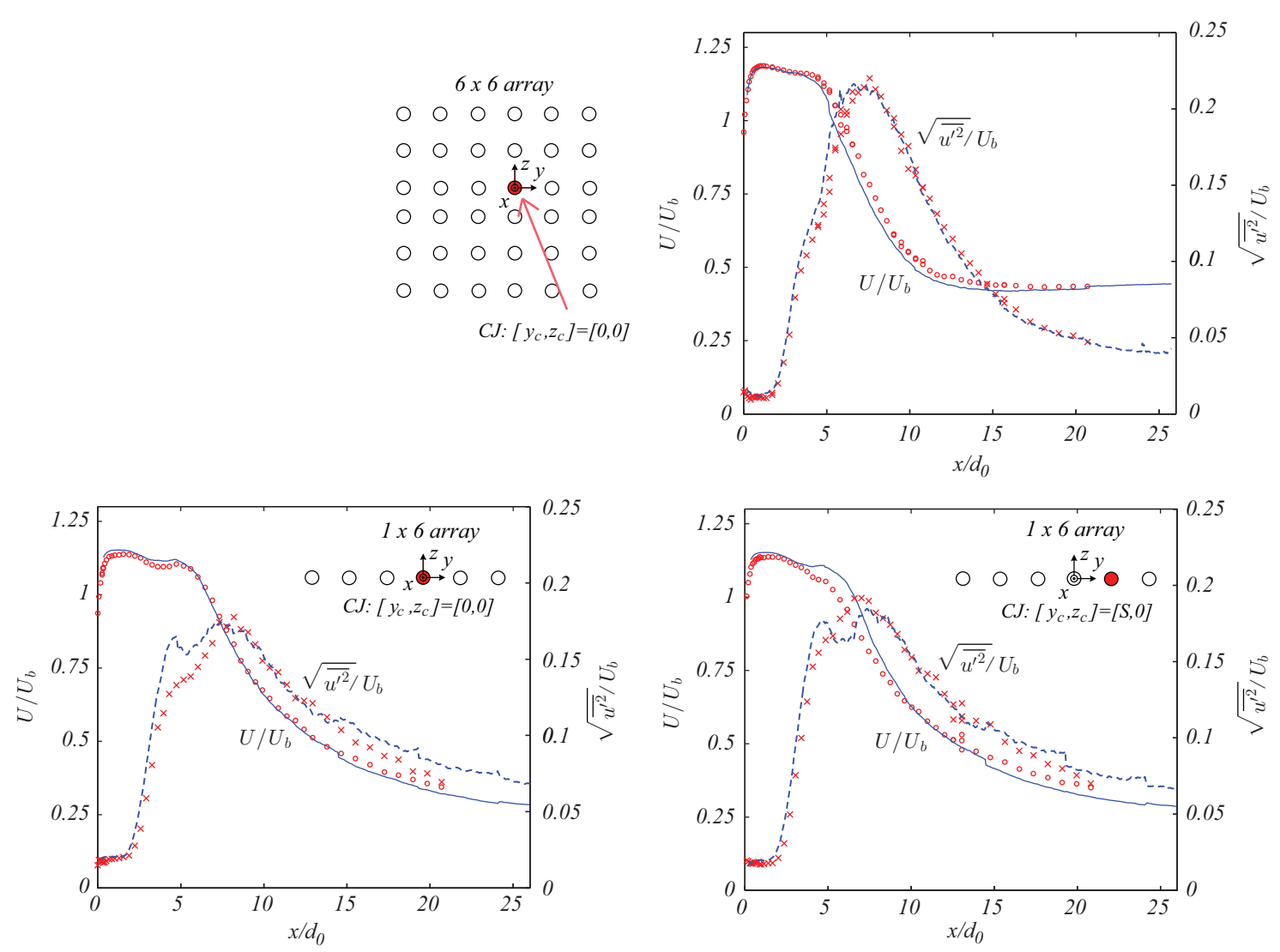

Figure 27 Dimensionless mean velocity in $x$-direction, $U / U_{b}$ (solid line and circle symbol) and the dimensionless RMS, $\sqrt{u^{\prime 2}} / U_{b}$, (dashed line and cross symbol) along the geometrical centerlines of one $C J$ in the $6 \times 6$ array (top) and two $C J \mathrm{~s}$ in the $1 \times 6$ array (bottom), measured by PIV (lines) and LDA (symbols) for $R e_{d}=3290$

\subsection{Comparison between single row of confluent jets and square confluent jet array}

This section provides a comparison of the flow field in the $1 \times 6$ and $6 \times 6$ array, based on results from PIV measurements. This part of the work aims at identifying principal differences between the two setups, where features that are only found in the confluent square array are identified for further analysis. The experimental results also provide new information on the behavior of a finite number of jets arranged in a single row. A complete description of the results from the experimental investigation of the $1 \times 6$ array, and a comparison with the $6 \times 6$ array, is found in Paper I. 


\subsubsection{Mean velocities}

In Figure 28 the in-plane $(z=0)$ dimensionless mean velocity magnitude, $\sqrt{U^{2}+V^{2}} / U_{b}$, for a row of jets located next to the array center in the $6 \times 6$ setup is compared with the velocity at the horizontal center plane of the $1 \times 6$ array. In the $6 \times 6$ array the $S J$ located at $y_{c}=2 S\left(y_{c} / d_{0}=5.64\right)$ bends toward the center of the array while there is no significant span-wise displacement for the $S J$ in the $1 \times 6$ setup. The spanwise displacement of the SJs in the $6 \times 6$ array causes a convergence followed by merging with the neighboring $C J$ s.

There is also merging of jets in the $1 \times 6$ setup, but this process is driven by jet spreading rather than the jets bending toward the array center. The inward bending of the jets in the $6 \times 6$ array affects both velocity decay and length of the merging region. The location of merging and combined points in the two setups is discussed in Section 8.5.3. As the jets in the $1 \times 6$ array do not bend towards the center, the converging region, which exists in for example twin jet configurations, does not occur in this setup. Instead it is more suitable to define an initial region upstream of the point where the jets begin to merge.

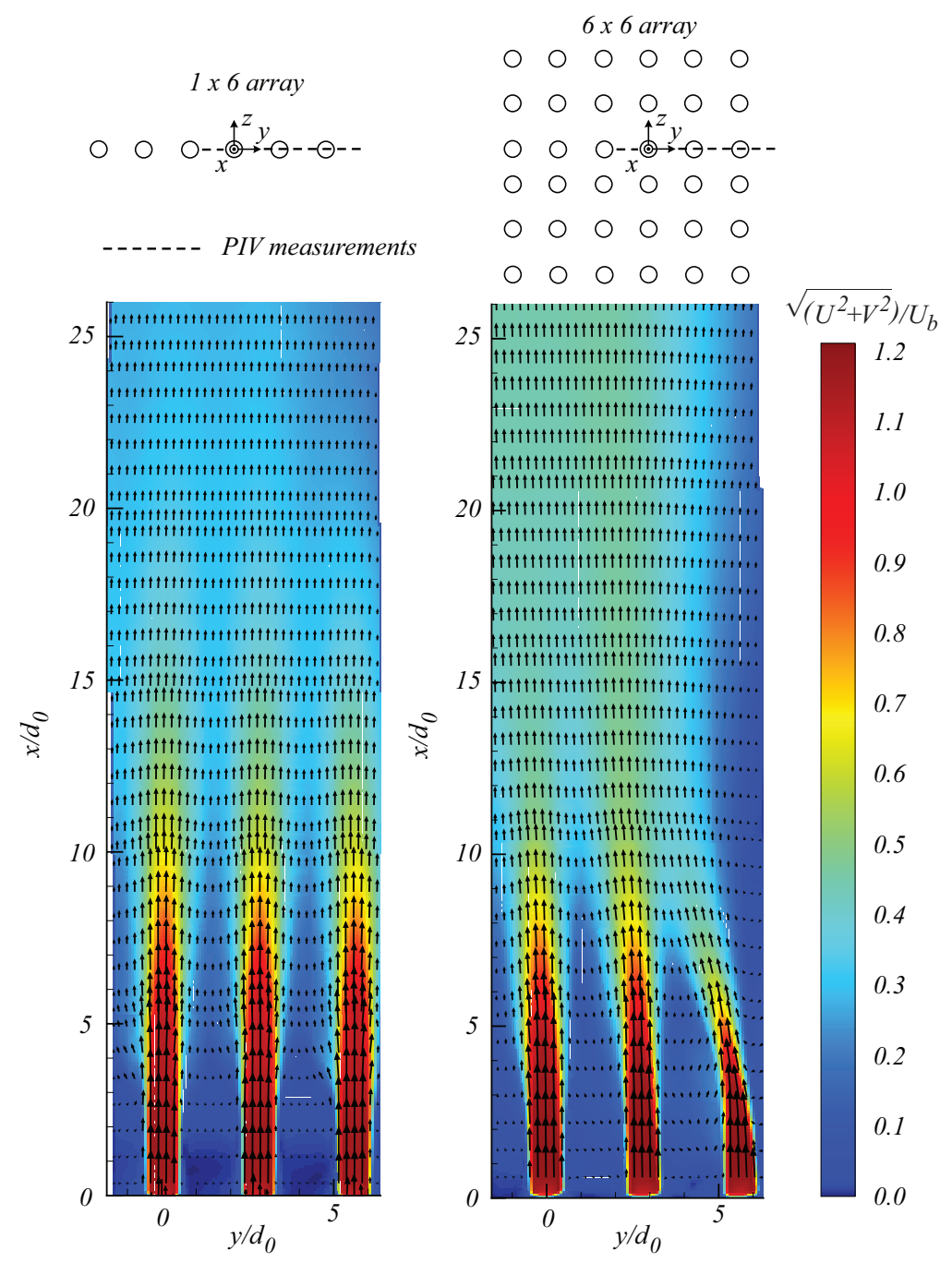

Figure 28 Contours of dimensionless mean velocity magnitude, $\sqrt{U^{2}+V^{2}} / U_{b}$, at the center plane of three jets in the $1 \times 6$ and $6 \times 6$ setup. 
In Figure 29 the development of the inverse of maximum stream-wise jet velocity, $U_{m} / U_{\text {max }, J}$ at three different Reynolds numbers $\left(R e_{d}=2125,3290\right.$ and 4555$)$ are shown for the same jets as in Figure 28. $U_{m}$ is the overall maximum stream-wise velocity of each jet, and varies between 1.14 and $1.19 U_{b}$, while $U_{m a x, I}$ is the maximum streamwise velocity of each jet at any given stream-wise $(x)$ position. Figure 29 also provides a comparison with a single round jet with the same nozzle shape at $R e_{d}=3461$ as reported by Ghahremanian and Moshfegh (2014), and the linear regression for a single round jet reported by Todde et al. (2009) at $R e_{d}=2700$.

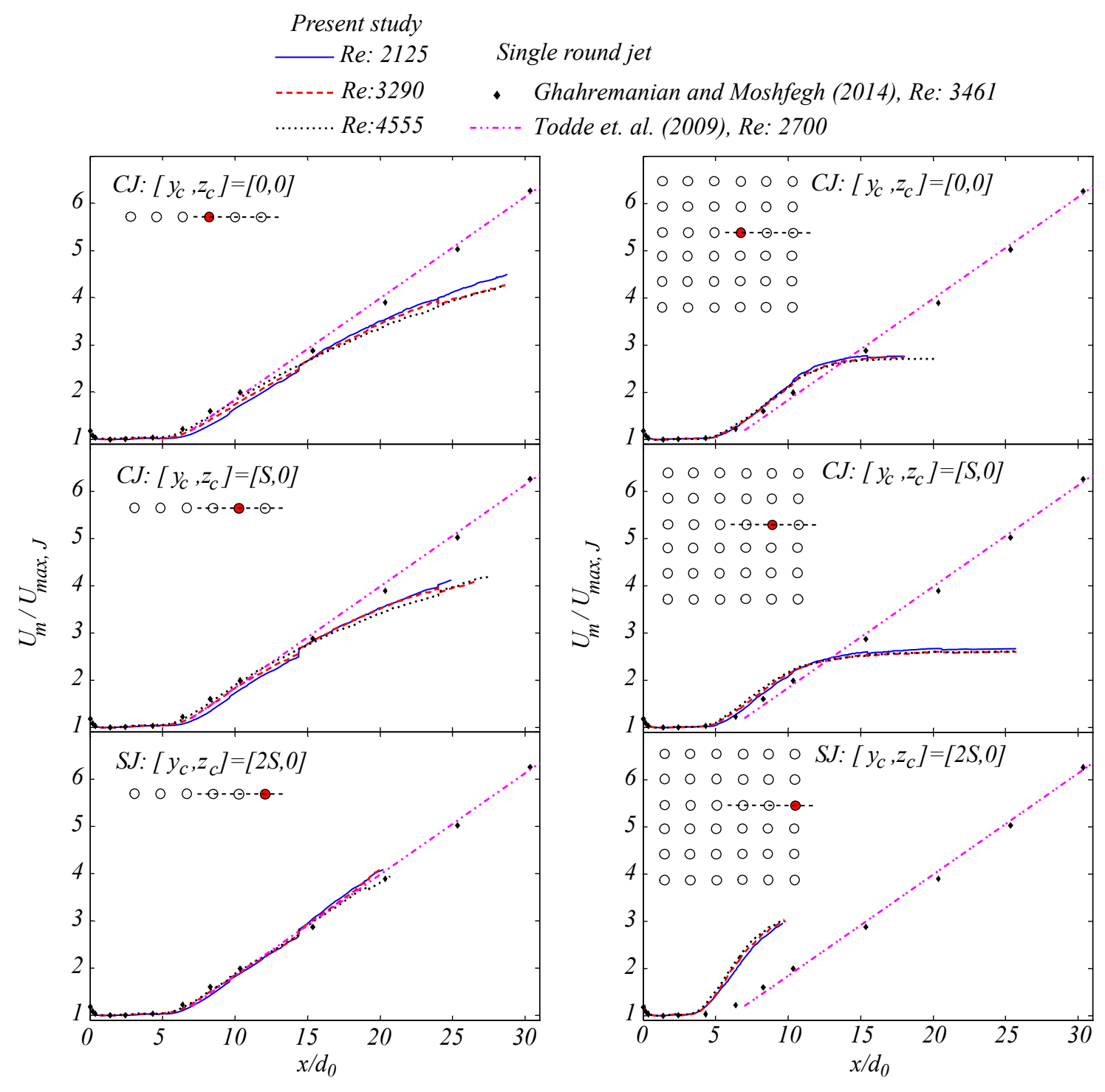

Figure 29 Development of inverse stream-wise maximum velocity, $U_{m} / U_{\max , J}$, showing the influence of jet position, array configuration and Reynolds number on maximum velocity decay. Comparison made with single round jet data by

Ghahremanian and Moshfegh (2014) and Todde et al. (2009).

There is a clear difference between both jet categories (CJs and $S J s)$ and the two setups. It is well known that $U_{m} / U_{\max , J}$ varies linearly with downstream distance $(x)$ in single round jets after an initial region, while for two-dimensional plane jets, $U_{m} / U_{\max , J}$ is proportional to $\sqrt{x}$. Knystautas (1962) showed that the velocity decay of a large number of coplanar jets will scale as two-dimensional plane jet after the jets combined 
together. To complement this finding it is shown in Paper I that the $C J \mathrm{~s}$ in the $1 \times 6$ setup will decay as $U_{m} / U_{\max , J} \propto \sqrt{x}$ already upstream of the combined region, while the velocity decay of the $S J$ will scale in the same way as a single round jet $\left(U_{m} / U_{\max , J} \propto x\right)$ up to the point where it combines with the adjacent $C J$ (see Figure 29). This leads to a higher velocity of the $C J \mathrm{~s}$ in the $1 \times 6$ setup compared to the $S J$. The $S J$ also combines earlier, around $x / d_{0}=20$, compared to $C J$ s which combine further downstream.

In the $6 \times 6$ setup the $S J$ decays much faster compared to a single round jet, and already around $x / d_{0}=10$ the $S J$ has combined with the neighboring jet. The $C J \mathrm{~s}$ also initially decay somewhat faster compared to a single round jet, which is not the case in the $1 \times 6$ setup. Further downstream the velocity decay of $C J$ s gradually ceases and $U_{m} / U_{m a x, I}$ reaches a constant value.

In Section 8.5.2 the region with constant maximum velocity, called the Confluent Core Zone, is discussed further. Please also note that a small degree of out-of-plane motion can be expected in the $6 \times 6$ setup. However, CFD simulations of the $6 \times 6$ setup (Paper IV) show the above observations still hold when $U_{m} / U_{\text {max }, J}$ is extracted from the complete three-dimensional flow field.

\subsubsection{Turbulent velocity field}

The lateral displacement in the $6 \times 6$ array also has implications on the development of turbulence. In Figure 30 the turbulent kinetic energy $(k)$ is presented for the two setups up to a stream-wise distance of $x / d_{0}=15$. $k$ is computed as $1 / 2\left(\overline{u^{\prime 2}}+\overline{v^{\prime 2}}\right) / U_{b}$, in order to avoid making assumptions regarding the third unknown Reynolds stress.

In the $1 \times 6$ setup the development of $1 / 2\left(\overline{u^{\prime 2}}+\overline{v^{\prime 2}}\right) / U_{b}$ is rather similar in all jets. For the $6 \times 6$ setup the $S J$ shows a substantially higher level of turbulence compared to the CJs. This is due to the lateral displacement. Bradshaw (1973) concluded that turbulent shear layers are strongly affected by mean flow curvature and the curvature is destabilizing when the angular momentum decreases in the direction away from the center of curvature as in the case of concave curvature. This effect is already considerable when the ratio of the shear layer thickness and the radius of curvature, $\delta / R$, exceeds 0.01 .

Shear layers facing away from the array center are the ones having a concave curvature. In the $6 \times 6$ array these shear layers are also the ones showing higher level of turbulence. In Paper II the development of the radius of curvature, $\delta / R$ was approximated for jets located along the diagonal of the $6 \times 6$ array. The results showed that $\delta / R$ reached values of approximately 0.03 to 0.09 , with increasing values of $\delta / R$ for jets further away from the array center. Also, normal production of $k$ due to the in-plane Reynolds stresses, $-\overline{u^{\prime 2}} \frac{\partial U}{\partial x}+\overline{-{\overline{v^{\prime}}}^{2}} \frac{\partial V}{\partial y}$, was shown to have a significant contribution to turbulent production for the $S J$ presented in Figure 30. As the additional contribution to the production of $k$ due to normal production relates to the bending of the jets, this observation is in agreement with the earlier finding. It is concluded that, as the jets merge, turbulence is promoted by mean flow curvature in the $6 \times 6$ array, while this effect is absent in the $1 \times 6$ array. 


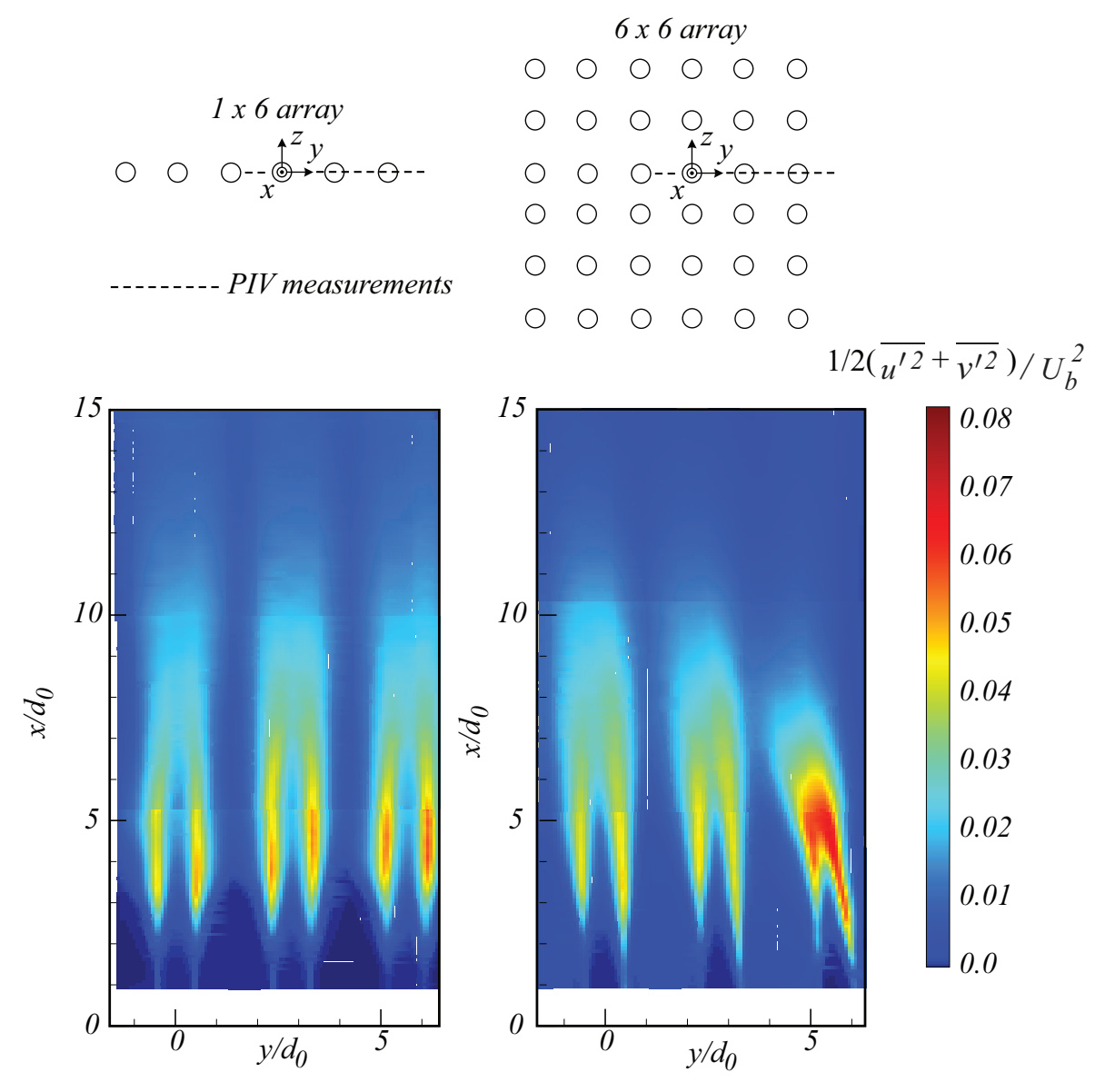

Figure 30 Dimensionless turbulence kinetic energy, $1 / 2\left(\overline{u^{\prime 2}}+\overline{v^{\prime 2}}\right) / U_{b}$, of a single row of $1 \times 6$ jets and a square $6 \times 6$ jet array.

\subsection{Validation of CFD model}

This section summarizes the validation of the CFD models performed in Paper III and IV, made through comparison with PIV data. A complete description of the CFD model validation is found mainly in Paper IV, but comparisons between PIV data and CFD results can also be found in Paper III, V and VI. The CFD model and implemented boundary conditions was described in Chapter 7.

\subsubsection{Influence of boundary conditions}

Experimental data from the PIV measurements was used for inlet boundary conditions for CFD simulations in both Paper III and IV. However, the method by which inlet boundary conditions were generated from the PIV data was refined from Paper III to Paper IV (see Section 7.1.2). Changes were also made to far-field boundaries and wall treatment (see Table 8). The change of far-field boundary conditions had no influence on the region of interest if the computational domain was made large enough. The change of wall treatment from standard to scalable wall functions only had small positive effects on the performance of the CFD model.

In Figure 31 the stream-wise velocity in the CFD simulations, using RSM, presented in Paper III and IV, is compared with PIV data at a number of downstream locations 
in two different planes, $z=0$ and $z=2 S$. The overall behavior is reproduced by both CFD models, but the more refined procedure for generating inlet boundary conditions in Paper IV provides substantial improvements compared to Paper III.
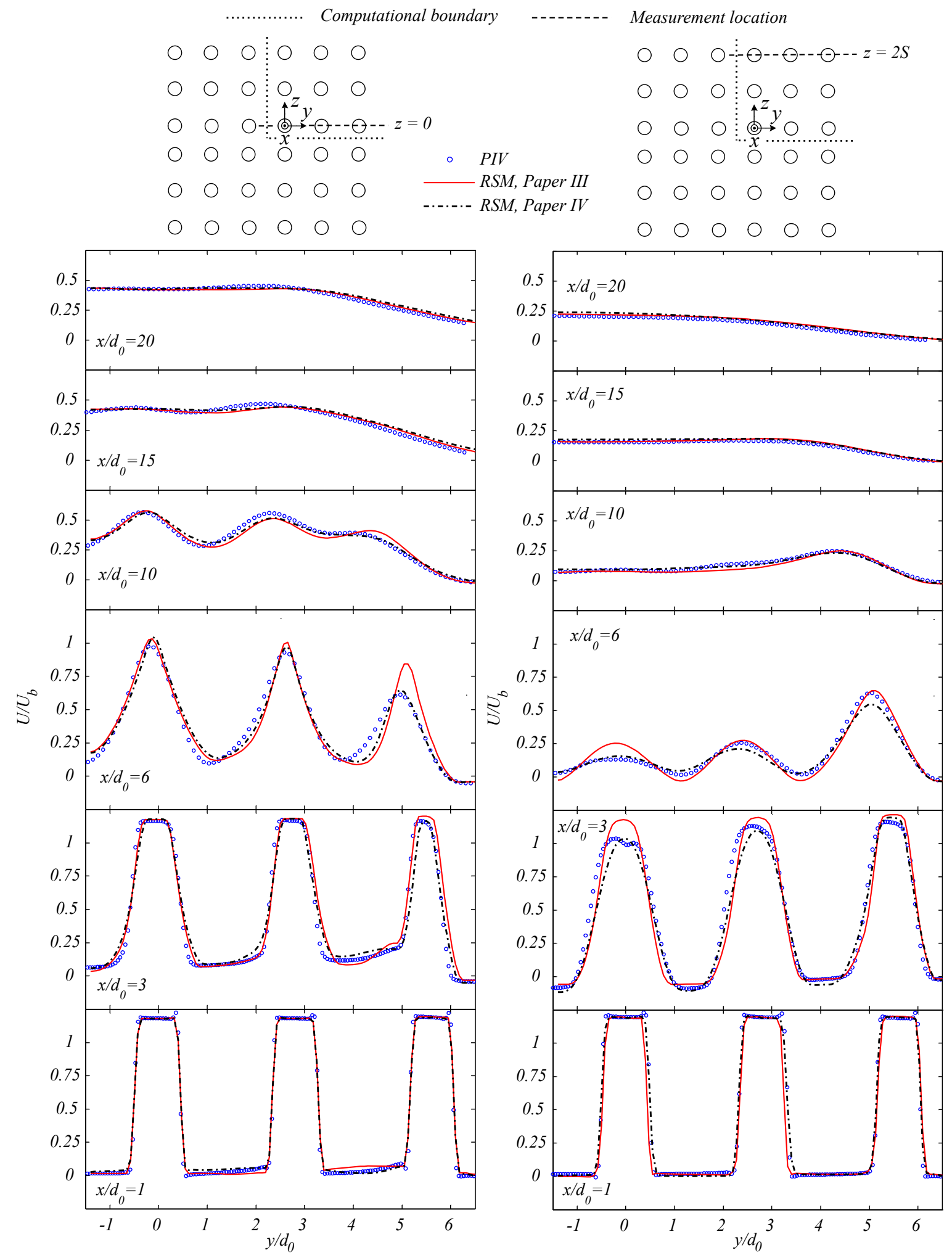

Figure 31 Comparison between PIV data and CFD simulations with RSM, using different inlet boundary conditions. 
In Paper III $U / U_{b}$ of $S J s$ are overpredicted in both measurement planes at $x / d_{0}=3$ and $x / d_{0}=6$, but in Paper IV the overprediction of $U / U_{b}$ for the $S J \mathrm{~s}$ is no longer present. Further downstream the two sets of boundary conditions produce similar results and the agreement with PIV data is recovered. Further simulations have shown that the improvements are due to removing the need to approximate $V$ and $W$ from the jets located at $z=0$, rather than the inclusion of the normal Reynolds stresses in the lateral directions $\left(\overline{v^{\prime 2}}\right.$ and $\left.\overline{w^{\prime 2}}\right)$.

It is concluded that when validating the computational model for the $6 \times 6$ jet array it is desirable to use boundary conditions from experimental data from all individual jets (as in Paper IV). The velocity in the lateral directions of individual jets in the array $(V$ and $W$ ) can have a substantial impact on the results, especially for the SJs, even though absolute values of $V$ and $W$ are small. As will be shown later, however, principal behavior and the characteristics of confluent jets arrays can be captured with CFD models even using uniform inlet profiles.

\subsubsection{Influence of turbulence model}

In Paper IV the performance of three turbulence models; the $k-\varepsilon s t d$., RNG $k-\varepsilon$ and RSM, was evaluated through comparison with PIV data (see Section 4.2. for description of turbulence models). In Figure 32 a comparison of CFD results for $U / U_{b}$, using different turbulence models, and PIV data are shown for the same locations as in Figure 31 in the preceding section. The PIV data shows that there is more rapid velocity decay of the $S J s$ in plane $z=0$, compared to $C J$ s. In the plane $z=2 S$, the velocity decay of $S J$ s is also reinforced by out-of-plane displacement, as the $S J \mathrm{~s}$ bend towards the center of the array. This results in low values of $U / U_{b}$ for the $S J \mathrm{~s}$ at $x / d_{0}=6$ in the plane $z=2 S$, followed by a recovery further downstream. Close to the nozzles the CoJ is associated with higher values of $U / U_{b}$ compared to $S J \mathrm{~s}$ in the same plane $(z=2 S)$. All investigated turbulence models reproduce all the abovementioned features with satisfactory agreement.

In Figure 33 the development of $U / U_{b}$ and the turbulent kinetic energy $\left(k / U_{b}^{2}\right)$ for the geometrical centerlines of jets located along the diagonal of the array $\left(y_{c}=z_{c}\right)$ are shown. The symmetry between the two span-wise directions has been used to approximate $k$ in the PIV data as $k=1 / 2\left(\overline{u^{\prime 2}}+2 \overline{v^{\prime 2}}\right)$. All three turbulence models predict an overall correct behavior of the jets' geometrical centerlines. The existence of a constant velocity region for the $C J$ at $\left[y_{c}, z_{c}\right]=[0,0]$, the small recovery in $U / U_{b}$ taking place for $C J$ at $\left[y_{c}, z_{c}\right]=[S, S]$, between $9 \lesssim x / d_{0} \lesssim 13$, and the rapid decay of $U / U_{b}$ for the CoJ are all found in both PIV and CFD results. The higher maximum values of $k$ for jets further away from the array center are also reproduced in the CFD results, although $k$ is associated with somewhat larger deviations compared to $U / U_{b}$. All investigated turbulence models however overpredict the length of the potential core, as the increase of $k$ starts earlier in the PIV data. The longer potential core causes a delay in the position at which mean velocity decay is initiated. In Paper VI the development of stream-wise turbulence intensity, $T_{U}$, defined according to Eq. (74), predicted by the $k-\varepsilon s t d$. model and PIV was compared at the same locations as 
in Figure 33. It was concluded that there is satisfactory agreement in the prediction of $T_{U}$, as long as the stream-wise velocity is not too low $\left(U / U_{b} \lesssim 0.1\right)$

$T_{U}=\frac{\sqrt{2 / 3 k}}{|U|}$

In Paper IV the ability of the RSM to predict values of the Reynolds stresses that are in agreement with PIV data was also investigated. The results showed that the RSM provides satisfactory agreement of stream-wise normal Reynolds stress $\left(\overline{u^{\prime 2}}\right)$ and shear stress $\left(\overline{u^{\prime} v^{\prime}}\right)$, but generally underpredicts the normal Reynolds stress in the span-wise direction $\left(\overline{v^{\prime 2}}\right)$. In general the RSM and $k-\varepsilon$ std. models provided smaller deviations between numerical and experimental results than the RNG $k-\varepsilon$ model. In terms of mean velocity RSM is not found to be superior to the less complex $k-\varepsilon s t d$. model.

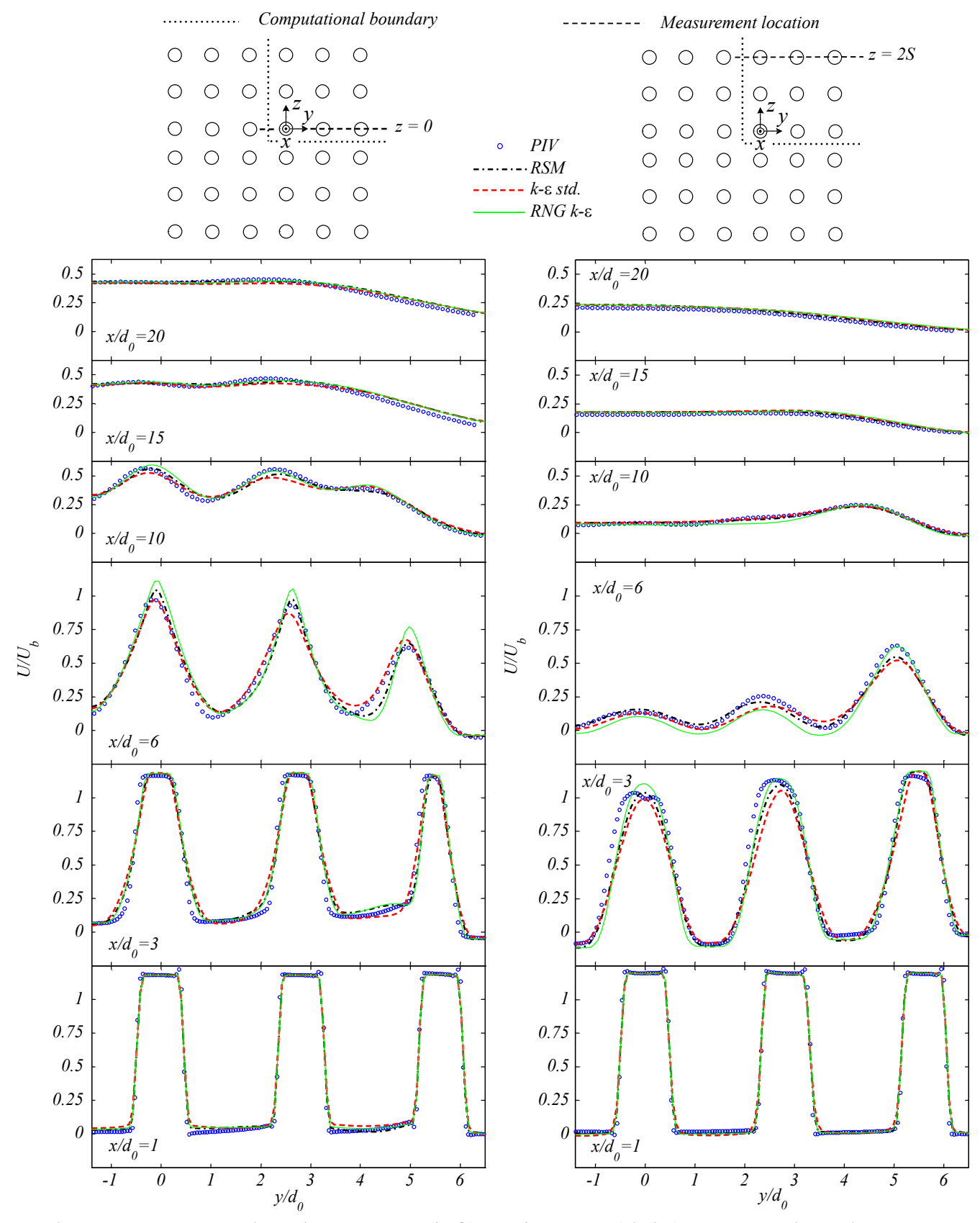

Figure $32 U / U_{b}$ at the plane, $z=0$ (left) and $z=2 S$ (right). Comparison between CFD results, using different turbulence models, and PIV data. 

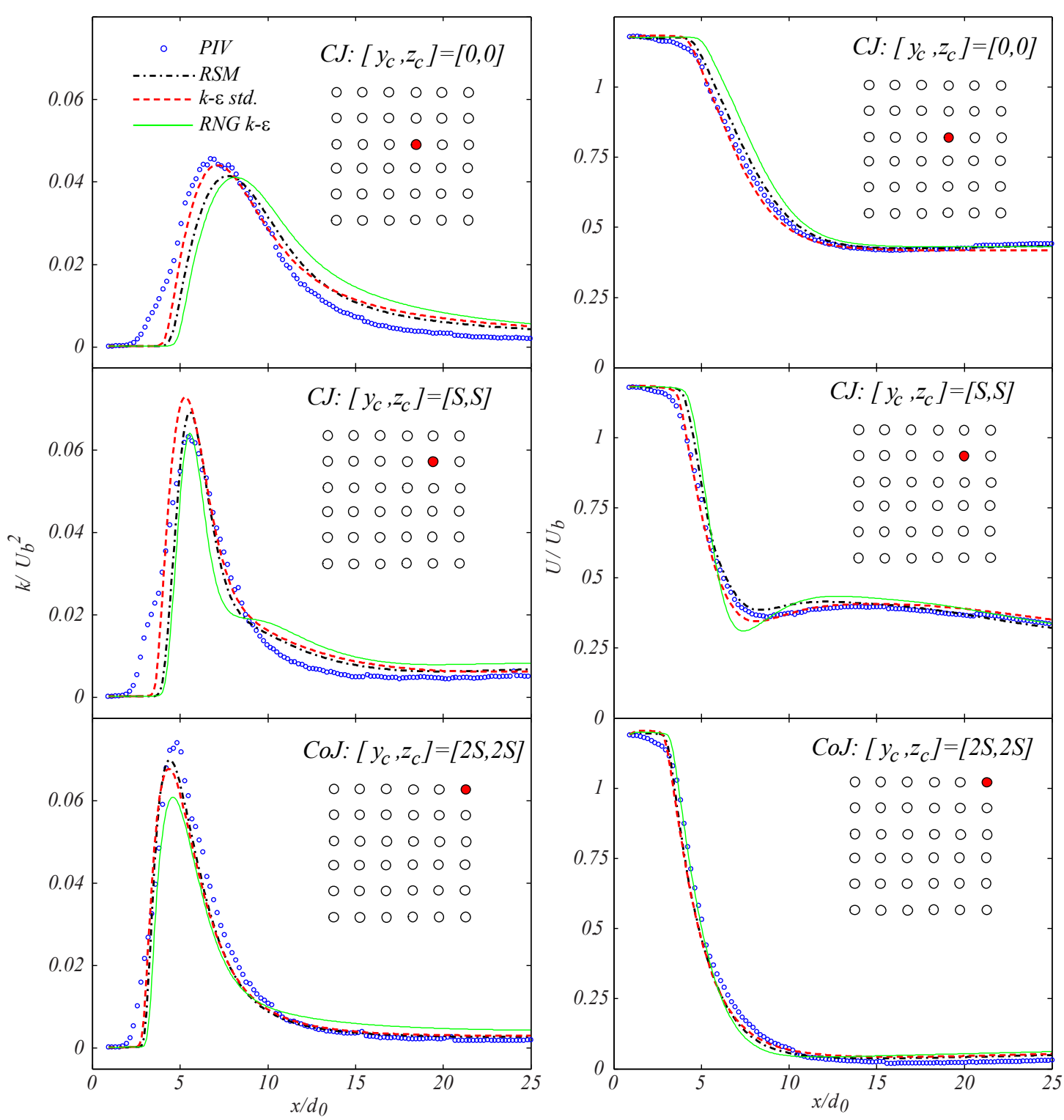

Figure $33 \mathrm{k} / U_{b}^{2}$ (left) and $U / U_{b}$ (right) along the geometrical centerlines of jets located along the diagonal symmetry plane of the array $\left(y_{c}=z_{c}\right)$. Comparison between CFD results, using different turbulence models, and PIV data.

\subsection{Flow pattern in confluent jet arrays}

This section summarizes important aspects of the flow field development derived from the investigations on the $6 \times 6$ confluent jet array (see geometrical properties in Section 6.2). The results in this section are mainly based on CFD simulations, but when possible PIV data are used to support the observations.

\subsubsection{Development upstream of combined region}

As seen in Figure 32 after a certain distance the confluent jets will form a flat velocity profile, in which individual jets may no longer be distinguished, commonly referred to as the jets are combined. Figure 34 presents PIV results of the development of maximum stream-wise velocity $\left(U_{\max , \mathrm{Z}} / U_{b}\right)$ in all planes where PIV measurements have been performed. Also added to the figure is the maximum velocity of a single 
round jet as measured by Ghahremanian and Moshfegh (2014). The figure shows that at around $x / d_{0} \approx 15$ the maximum stream-wise velocity in planes located in the central parts of the array $(|z| \leq S)$ in almost the same and rather constant. At $z=1.5 S$ and $z=2 S$ the maximum stream-wise velocity is lower due to the contraction of the jet array. This indicates that at $x / d_{0}=15$ the confluent jets are combined in the investigated setup.
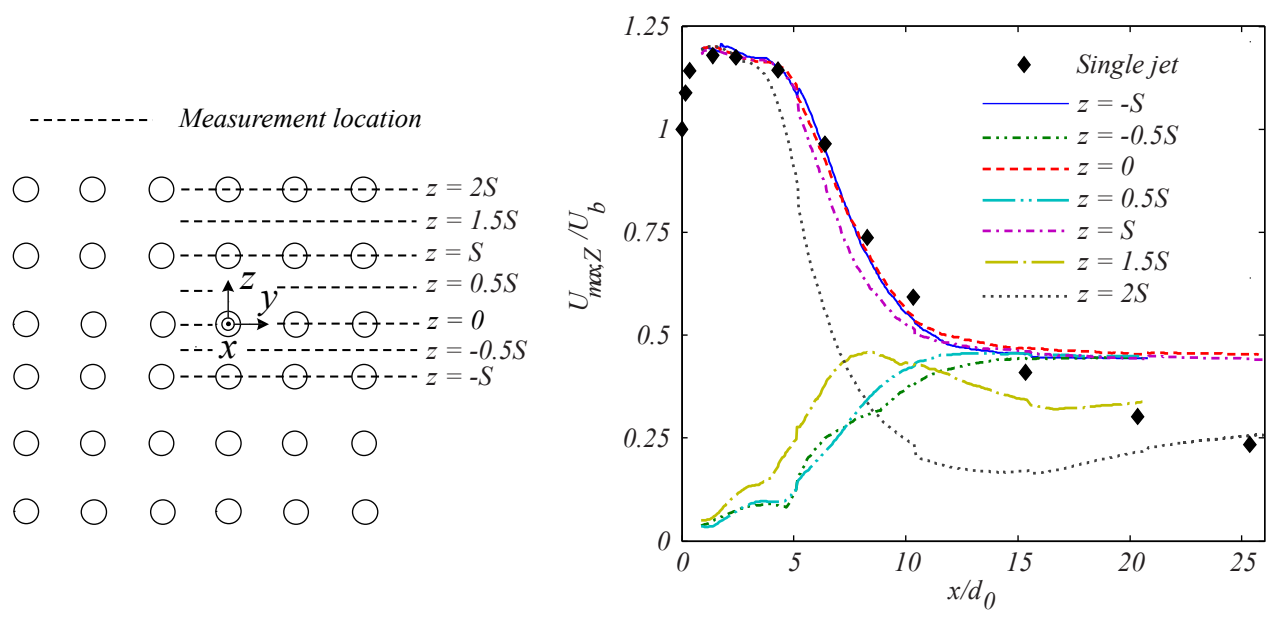

Figure 34 Maximum velocity in the stream-wise $(x)$ direction at different PIV measurement planes $\left(R e_{d}=3290\right)$. Comparison with maximum velocity for single round jet measured by Ghahremanian and Moshfegh (2014).
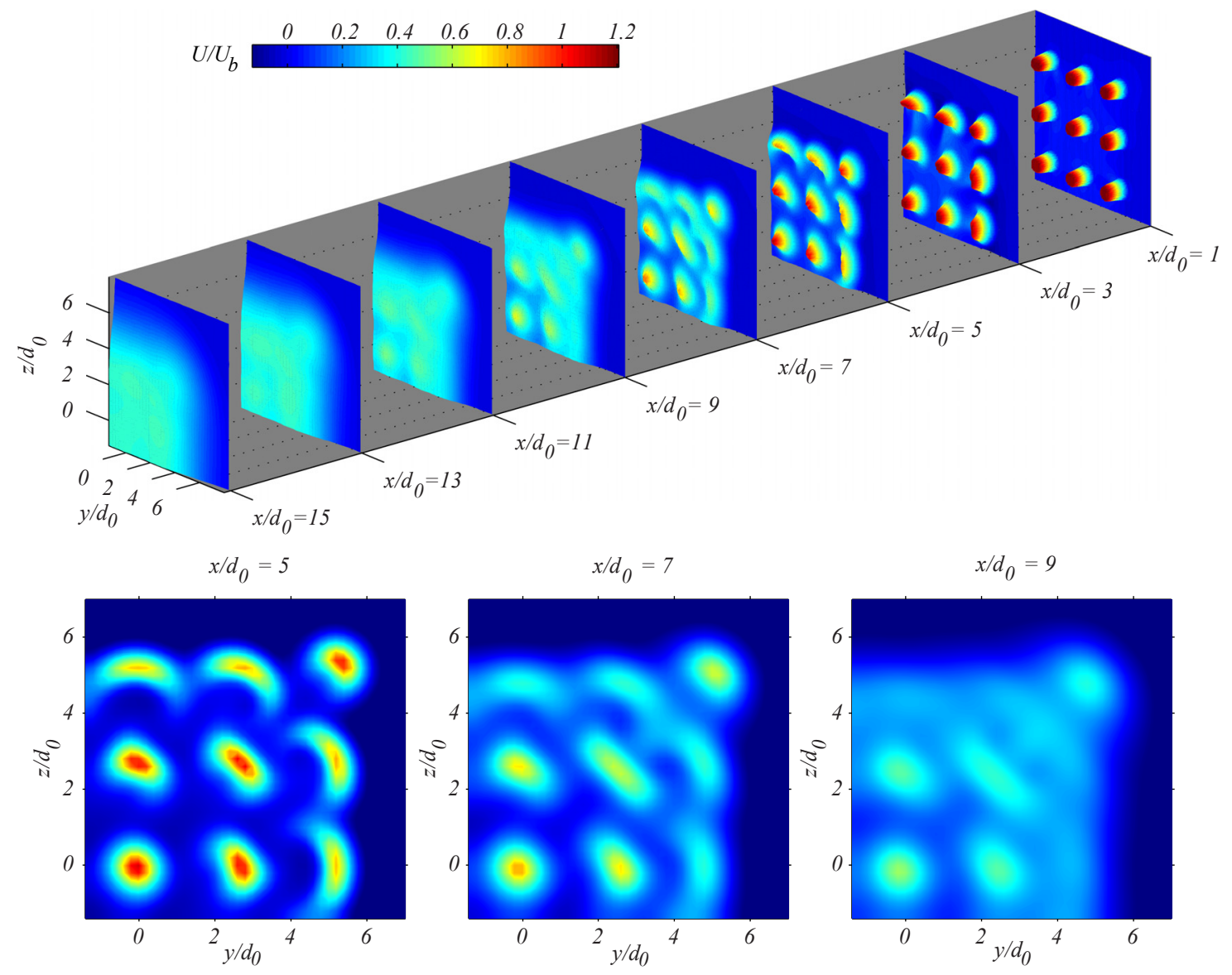

Figure $35 U / U_{b}$ at different downstream cross-sectional planes predicted by RSM. 
In Figure 35 the development of $U / U_{b}$ upstream of the combined region, predicted with RSM, is presented. The results show that the SJs in the $6 \times 6$ array are not only displaced towards the center of the array, they are also deformed, adapting a kidneyshaped form, shortly after the nozzle exit. The lateral jet displacement and jet deformation leads to a shorter potential core, higher turbulence level, and faster velocity decay of $S J$ s compared to $C J$ s. The existence of jet deformations is supported by the PIV results in Figure 32, as the PIV measurements at $x / d_{0}=3$ exhibit much broader velocity profiles of the $S J \mathrm{~s}$ at $z=2 S$ compared to the narrow jet profile of the SJ at $z=0$.

The CoJ is less affected by jet interaction than SJs. The CoJ will preserve its maximum velocity better than $S J$ s and have a longer potential core, which is more comparable to CJs. The CJs are also deformed but generally less than SJs. Especially the CJs next to the array center preserve a relatively round shape.

\subsubsection{Combined jet development and Confluent Core Zone (CCZ)}

The bending of the jets in the array towards the array center, as they interact with each other before being combined, influence the shape and spreading rate of the combined jet. Figure 36 shows the horizontal displacement of the central streamlines of the CoJ and the $S J$ located at $\left[y_{c}, z_{c}\right]=[2 S, 0]$. It may be noted that the reported streamlines are located in the planes $z=0$ or $z=2 S$ in order to enable comparison with the planar PIV data.
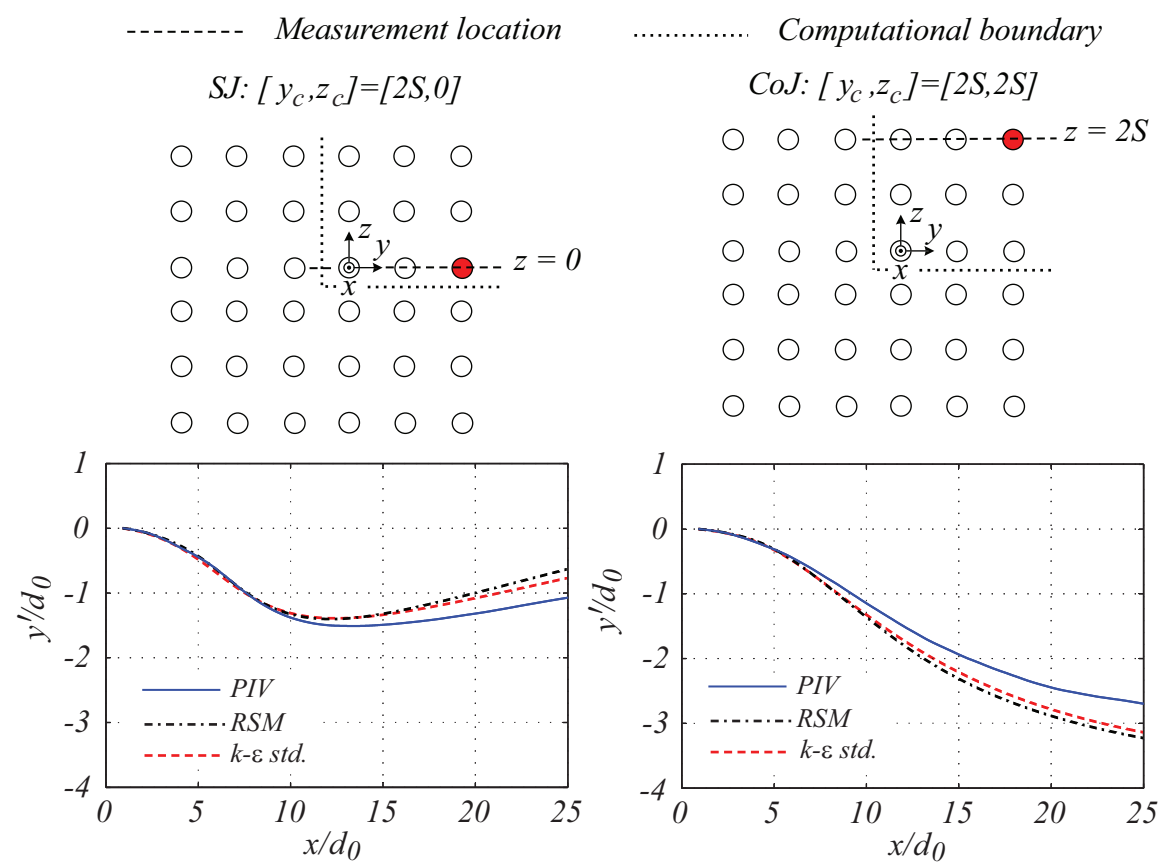

Figure 36 Lateral in-plane displacement in the $y$-direction of central streamlines for two different jets.

The results in Figure 36 show that initially both the SJ and CoJ bend towards the array center. Around $x / d_{0}=13$ the inward bending of the $S J$ ceases and the residue of the SJ, which is now combined with neighboring jets, starts to spread outwards. For the CoJ the inward bending progresses downstream of $x / d_{0}=25$, which is the maximum 
downstream distance of the PIV measurements. The same behavior is found in both PIV and CFD results.

Commonly the half-width, $r_{0.5} / d_{0}$, is used to describe the jet width (see definition in Section 2.1). In Figure 37 the half-width along the diagonal $(y=z)$ and the horizontal symmetry plane $(z=-0.5 S)$ is presented from $x / d_{0}=10$ up to $x / d_{0}=150$ for the $6 \times 6$ jet array. Upstream of $x / d_{0}=10$ there are regions in-between jets with low velocity, which makes $r_{0.5} / d_{0}$ only relevant for individual jets. Also added to the figure are contour plots of $U / U_{b}$ overlaid by vector plots of $V$ and $W$ at three downstream locations. The presented results are computed with the $k-\varepsilon s t d$. model.
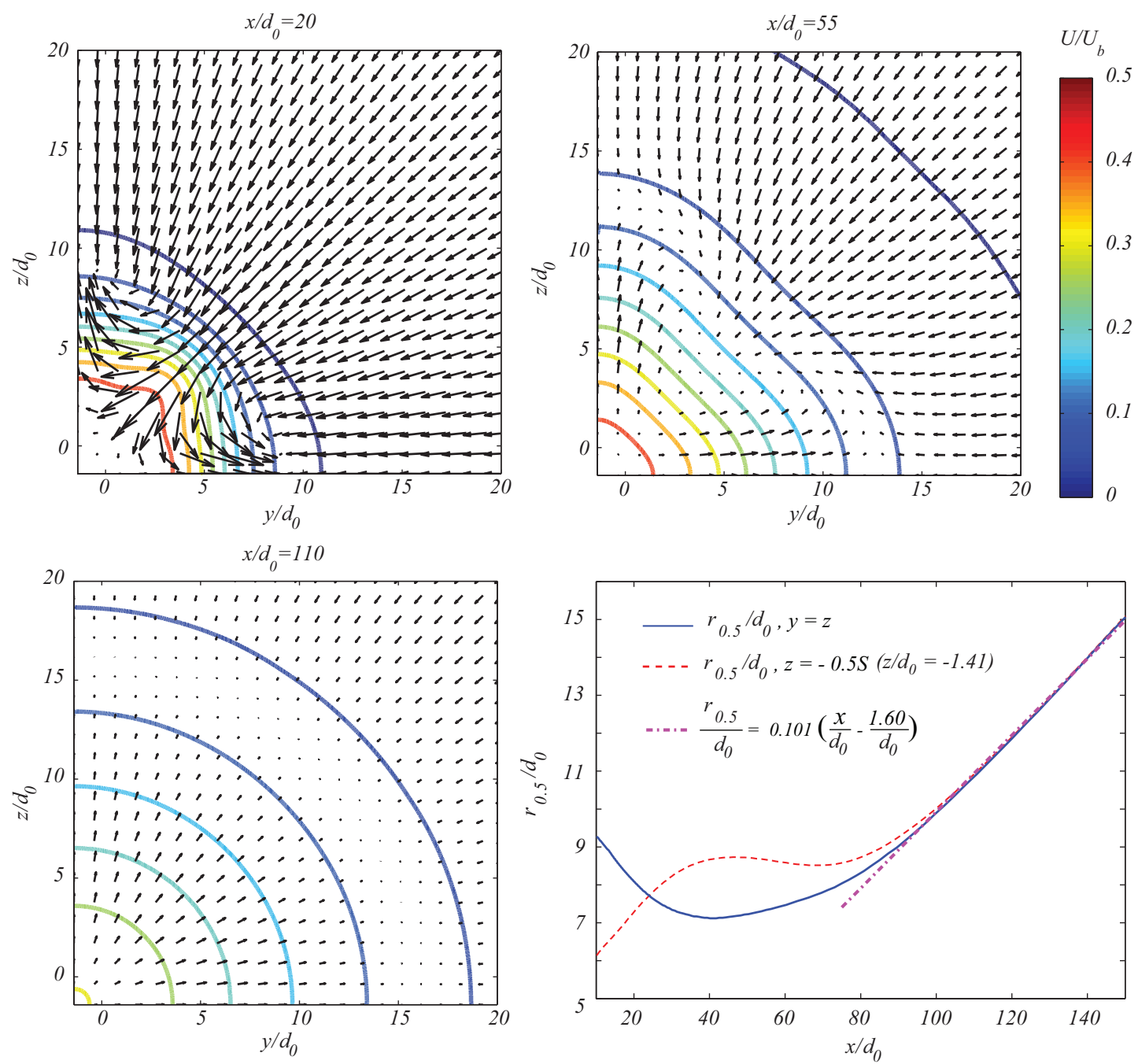

Figure 37 Development of the combined jet shape. Cross-sectional contour plots of $U / U_{b}$ overlaid by the velocity field in the lateral directions $(V$ and $W)$ and development of $r_{0.5} / d_{0}$ along $y=z$ and $z=-0.5 S$.

Three stages of the development can be identified for the combined jet upstream of $x / d_{0}=150$. At first the combined jet has a square shape and is therefore wider in the diagonal direction. The combined jet is spreading along the horizontal and vertical symmetry planes, while there is contraction along its diagonal, i.e., $r_{0.5} / d_{0}$ at $z=-0.5 S$ is increasing, while $r_{0.5} / d_{0}$ along $y=z$ is reducing. This process is also reflected by the vector plot in Figure 37 at $x / d_{0}=20$. This behavior causes a flattening of the 
combined jet along its diagonal and at approximately $x / d_{0} \approx 24$ axis-switching takes place as the combined jet half-width is smaller along the diagonal compared to the horizontal symmetry plane. This behavior brings to mind a single square jet, which may also exhibit axis-switching between the diagonal and horizontal direction (see e.g. Grinstein et al. (1995)).

Further downstream the lateral velocity will also change direction along the diagonal, and $r_{0.5} / d_{0}$ start to increase also in the diagonal direction. At the same time there is a reduction of $r_{0.5} / d_{0}$ along the horizontal symmetry plane. This is due to widening of the jet shear layers around a core region of constant stream-wise velocity and not due to jet contraction. Comparing the profile of $U / U_{b}$ at $x / d_{0}=20$ and $x / d_{0}=55$ it is seen that the width of the shear layers is growing, and that there is a region of constant velocity at the jet core which is reducing with downstream distance. This causes the reduction in $r_{0.5} / d_{0}$ at $z=-0.5 S$. Finally the shear layers penetrate all the way to the center of the combined jet, the jet turns axisymmetric, and the maximum stream-wise velocity starts to decay again. As the combined jet turns axisymmetric the spreading rate becomes linear (see Figure 37) and attains a value which is similar to a single round jet.

As already discussed a zone with uniform stream-wise velocity forms as the jets are combined. In Figure 38 the development of maximum stream-wise velocity of all jets in the array, $U_{\max } / U_{b}$, is presented. Also added to the figure is development of minimum turbulence intensity, $T_{U, \text { min }} . T_{U, \text { min }}$ is based on the local stream-wise velocity and is computed according to Eq. (75).

$T_{U, \min }=\min \left(\frac{\sqrt{2 / 3 k}}{|U|}\right)$

The figure shows that around $14 \lesssim x / d_{0} \lesssim 20$ the maximum stream-wise velocity will gradually approch a constant value of approximately $0.42 U_{b}$. $U_{\text {max }} / U_{b}$ remains constant up to $x / d_{0} \approx 65$, where the decay in maximum stream-wise velocity progresses. Within the zone of uniform stream-wise velocity the minimum turbulence intensity decreases to approximately $T_{U, \min }=0.08$.

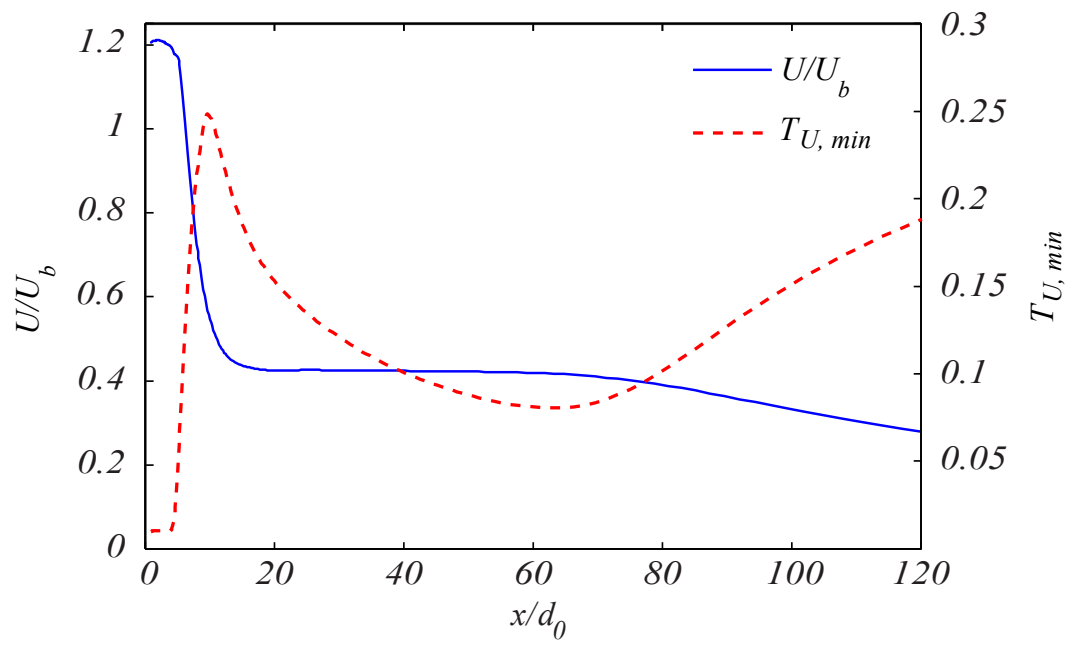

Figure 38 Development of maximum stream-wise velocity, $U_{\max } / U_{b}$ and minimum turbulence intensity $T_{U, \min }$. 
In Figure 39 iso-contours of $U / U_{b}=0.41$ and $U / U_{b}=0.43$ are presented, in order to illustrate the region with constant $\left(U / U_{b} \approx 0.42\right)$ stream-wise velocity. Upstream of $x / d_{0} \approx 14$ these contours are located close to each other in the shear layers surrounding the jets. Between $x / d_{0}=14$ and $x / d_{0}=18$ the velocity falls below $U / U_{b}=$ 0.43 for all $C J$ s. In the center of the array a zone forms, which extends relatively largely in the lateral directions, having a velocity between $0.41 \leq U / U_{b} \leq 0.43$. The zone with uniform velocity reduces in size with downstream distance resulting in a conical shaped region of constant velocity. Figure 39 also shows iso-contours where turbulence intensity, $T_{U}$, is $35 \%$ and $10 \%$. The region between jets, before they combine, is highly turbulent, but as the jets combine turbulence intensity reduces and in the downstream part of the zone with uniform $U / U_{b}, T_{U}$ is lower than $10 \%$. The constant stream-wise velocity, the conical shape due to the gradual penetration of the surrounding shear layers, and the low level of turbulence all to some extent are reminiscent of a potential core in a single round jet. Therefore this zone of development was called Confluent Core Zone (CCZ). In Section 8.6.4 it is also shown that the proper scaling length will change from inlet diameter $\left(d_{0}\right)$ to effective source diameter $\left(d_{s}\right)$, within the CCZ. For further discussion on the CCZ and development of the combined jet please see Paper V and VI.

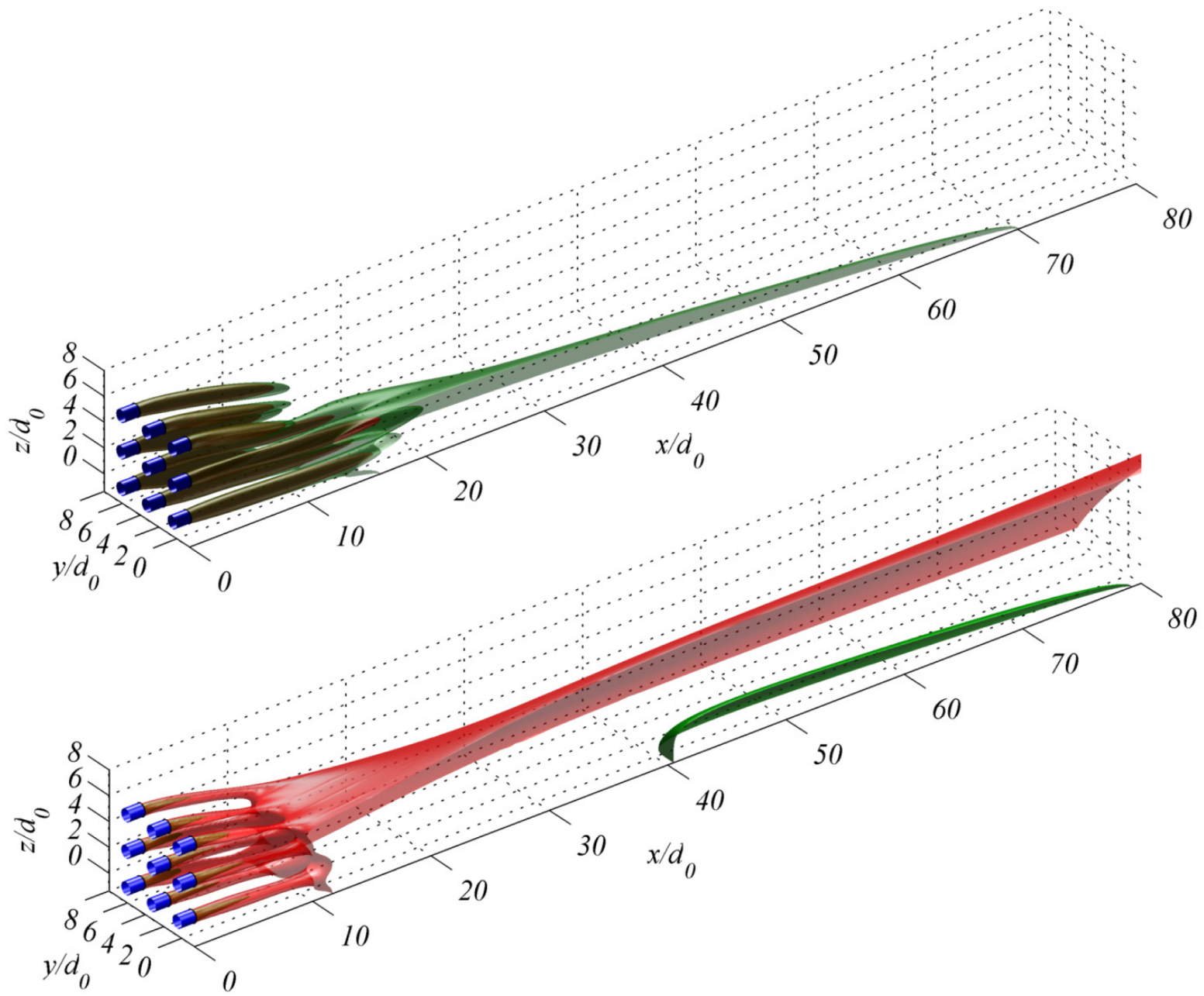

Figure 39 Illustration of CCZ prevalence. Top: iso-contours of $U / U_{b}=0.41$ (green) and $U / U_{b}=0.43$ (red) Bottom: iso-contours of $T_{U}=0.35$ (red) and

$$
T_{U}=0.1 \text { (green) }
$$




\subsubsection{Characteristic points and regions of development}

As discussed in Section 2.2 plane twin jets have three regions of development: converging, merging and combined region. All three processes that have given name to these regions are found in the $6 \times 6$ square confluent jet array, while converging is absent in the investigated row of $1 \times 6$ jets (see Section 8.3). The transition between converging and merging region, and between merging and combined region, is defined by the location of merging point $\left(x_{m p} / d_{0}\right)$ and combined point $\left(x_{c p} / d_{0}\right)$. This section summarizes the findings regarding the locations of these characteristic points in the investigated $6 \times 6$ confluent jet array. The location of characteristic points has been estimated either from two-dimensional PIV data as in Paper I and II, or from CFD results covering the whole flow field as in Paper IV and V.

The strong recirculation zone that occurs between twin plane jets, does not exist in the investigated $6 \times 6$ jet array, which only has limited amount of back flow between jets. Therefore the definition of $x_{m p} / d_{0}$ as the location of the free stagnation cannot be used. Instead the definition proposed by Vouros and Panidis (2008) is employed. Their definition was originally intended for twin round jets and states that $x_{m p} / d_{0}$ is the location where the minimum stream-wise velocity between the jets, $U_{\text {min, }}$, exceeds $10 \%$ of the maximum stream-wise velocity in the flow field, i.e., $U_{\min , J}=0.1 \max (U)$.

In general $x_{c p} / d_{0}$ is defined as the location where two jets share a common maximum in stream-wise velocity, i.e., where there is no longer a local minimum in $U / U_{b}$ between two jets. This definition was not a robust criterion for $C J \mathrm{~s}$ in the $6 \times 6$ array. Therefore the adjusted combined point $\left(x_{a c p} / d_{0}\right)$, defined as the point where the minimum velocity between two jets does not fall below $90 \%$ of the maximum velocity for the jet under consideration $\left(U_{\max , J}\right)$, was introduced in Paper IV. In Figure 40 the development of $U_{\text {min,J }} / U_{b}$ and $U_{\max , J} / U_{b}$ is shown for the $C J$ located at $\left[y_{c}, z_{c}\right]=[0,0]$ and the $S J$ at $\left[y_{c}, z_{c}\right]=[2 S, 0]$ in the $6 \times 6$ array. The figure contains both PIV and CFD results. Due to the planar PIV data, the results are limited to the plane $z=0$. The figure also reports the span in which PIV and CFD results, using either $k-\varepsilon$ std. model or RSM, predict merging and adjusted combined point locations for the two jets.

For the CJ the CFD simulations predict somewhat lower values of both $x_{m p} / d_{0}$ and $x_{a c p} / d_{0}$, compared to PIV. As there is good agreement between PIV and CFD results for $U_{\max , J} / U_{b}$ this relates to an overprediction of $U_{\text {min, } J} / U_{b}$ downstream of $x / d_{0}=5$ in the CFD results. Due the creation of the CCZ $U_{\text {min,J }} / U_{b}$ and $U_{\text {max }, J} / U_{b}$ approach each other slowly for the $C J$ in the downstream part of the merging region, which causes the previously mentioned difficulties in accurately determining $x_{c p} / d_{0}$ for $C J \mathrm{~s}$.

For the $S J U_{\text {min,J }} / U_{b}$ and $U_{\max , J} / U_{b}$ approach each other more rapidly, which facilitates the determination of $x_{c p} / d_{0}$. On the other hand, the behavior of $U_{\text {min, },} / U_{b}$ is more complex for the $S J$. Initially, $U_{m i n, J} / U_{b}$ rapidly increases to a level approximately equal to the merging point criteria, then slightly falls back, before merging is resumed in full. The PIV measurement and $k-\varepsilon$ std. model barely miss the merging point criteria around $x / d_{0}=3$, while the RSM has a local maximum in $U_{\text {min, }} / U_{b}$ at this location that 
meets the criteria of $x_{m p} / d_{0}$. Further downstream, during the main part of merging, the RSM however shows excellent agreement with PIV data of $U_{\min , J} / U_{b}$ for the SJ.

The development of $U_{\text {min, }} / U_{b}$ shows the difficulty in defining a specific point where merging between jets is initiated in the investigated setup. Nevertheless the definition of $x_{m p} / d_{0}$ by Vouros and Panidis (2008) seems to be a suitable alternative as $U_{m i n, J} / U_{b}$ starts to grow more rapidly directly downstream of $x_{m p} / d_{0}$ for both the $C J$ and the $S J$.
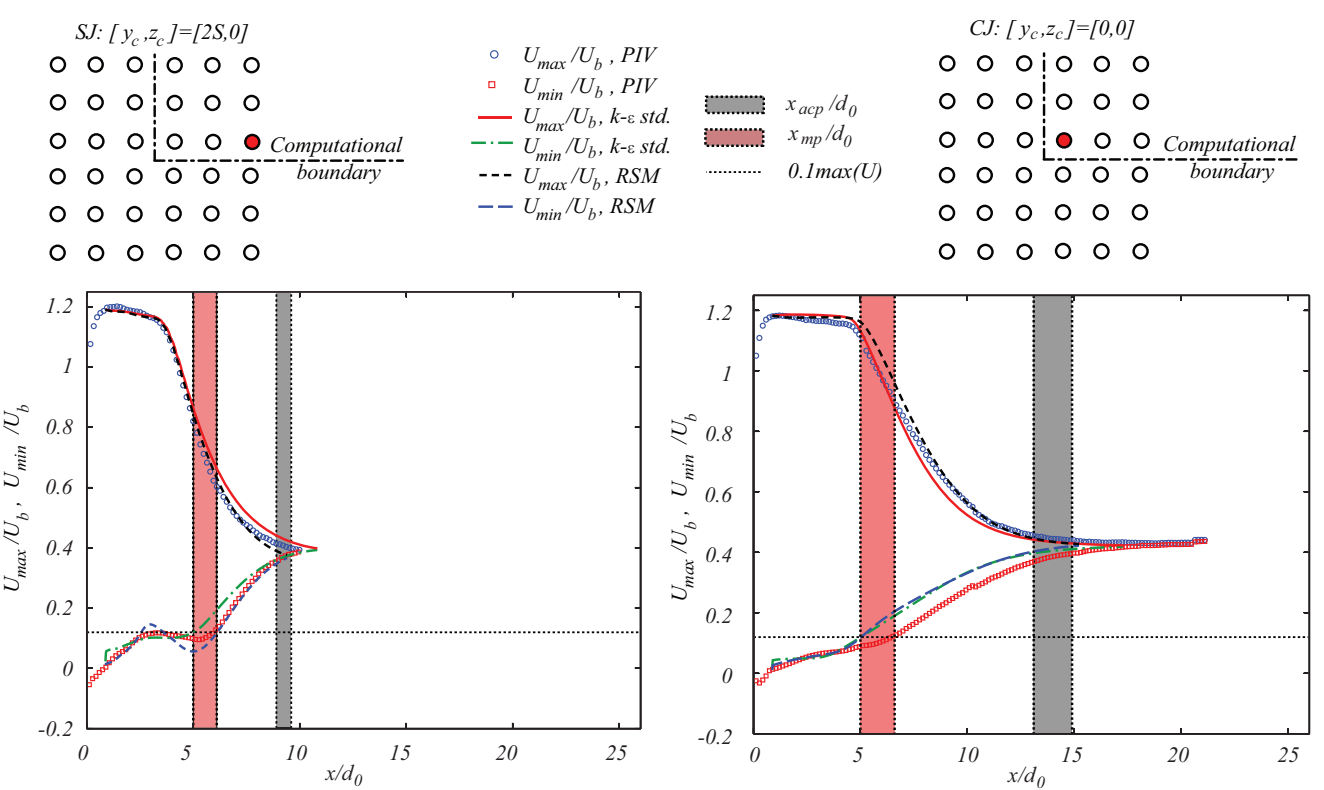

Figure 40 Development of $U_{\text {max, }} / U_{b}$ and $U_{\text {min, }} / U_{b}$, merging point criteria by Vouros and Panidis (2008) and predictions of $x_{m p} / d_{0}$ and $x_{a c p} / d_{0}$ for the $6 \times 6$ array in plane $z=0 . R e_{d}=3290$

Numerical values of $x_{m p} / d_{0}, x_{a c p} / d_{0}$ and $x_{c p} / d_{0}$ for the two jets plotted in Figure 40 are presented in Table 10. Also added to the table are results based on PIV measurements for the same jets in the $1 \times 6$ array and location of the characteristic points in the $6 \times 6$ array, computed from CFD results and considering merging and combining in the whole flow field, not only in the plane $z=0$. In both the $6 \times 6$ and $1 \times 6$ setup there is a clear difference in $x_{a c p} / d_{0}$ and $x_{c p} / d_{0}$ between the two investigated jets. The $C J$ combine later in both setups. Comparing the two setups with each other, both jets combine earlier in the $6 \times 6$ setup.

The start of merging region $\left(x_{m p} / d_{0}\right)$ for the $S J$ is directionally dependent due to jet deformation. When all possible directions of jet merging are considered, the CFD simulations predict an upstream location of $x_{m p} / d_{0}$ for the $S J$ in the $6 \times 6$ array compared to other jets.

Comparison between $x_{m p} / d_{0}$ and $x_{c p} / d_{0}$ in the investigated confluent jet arrays with reported values in other jet configurations has been made in Paper I and II. Reported locations of characteristic points in the literature for twin plane jets, twin round jets and a single row of jets, having similar dimensionless jet spacing $\left(S / d_{0}\right.$ or $\left.S / w\right)$, are also found in Tables 2, 3 and 4 in Chapter 2. 
In the $1 \times 6$ array $x_{c p} / d_{0}$, is reduced for jets at the edges of the row $(S J \mathrm{~s})$ compared to both twin round jets and a single row with a large number of round jets. For CJs in the $1 \times 6$ array the location of $x_{c p} / d_{0}$ seems to coincide fairly well with reported data for twin round jets and a single row with a larger number of round jets. For twin or multiple round jets there is limited data of $x_{m p} / d_{0}$. Comparison with data extracted from the investigation on twin round jets by Harima (2001) however shows that the values of $x_{m p} / d_{0}$ in the $1 \times 6$ array $\left(x_{m p} / d_{0} \approx 5\right)$, seems similar to twin round jets. In comparison with twin plane jets both $x_{m p} / d_{0}$ and $x_{c p} / d_{0}$ are located further downstream in the $1 \times 6$ array. The more open structure of the $1 \times 6$ array leads to a longer merging region compared to twin plane jets.

Table 10 Stream-wise location of merging point $\left(x_{m p} / d_{0}\right)$, adjusted combined point $\left(x_{a c p} / d_{0}\right)$ and combined point $\left(x_{c p} / d_{0}\right), R e_{d}=3290$.

\begin{tabular}{|c|c|c|c|c|c|}
\hline Jet & Configuration & Source & $x_{m p} / d_{0}$ & $x_{a c p} / d_{0}$ & $x_{c p} / d_{0}$ \\
\hline \multirow{6}{*}{$\begin{array}{c}S J \\
{\left[y_{c}, z_{c}\right]} \\
= \\
{[2 S, 0]}\end{array}$} & $\begin{array}{l}\text { I) Paper I } \\
1 \times 6\end{array}$ & PIV & $5.2^{\mathrm{I})}$ & 17.7 & $20.1^{\mathrm{I}}$ \\
\hline & \multirow{3}{*}{$\begin{array}{c}{ }^{\mathrm{II}} \text { Paper II } \\
6 \times 6, \text { planar data } z=0\end{array}$} & PIV & $5.9^{\mathrm{II})}$ & 9.2 & $9.9,(10)^{\mathrm{II})}$ \\
\hline & & $k-\varepsilon s t d$. & 5.0 & 9.6 & 10.8 \\
\hline & & RSM & $2.6^{* *}, 6.1^{* *}$ & 8.9 & 9.4 \\
\hline & \multirow{2}{*}{$\begin{array}{c}\text { Paper IV: } \\
6 \times 6 \text {, complete flow field }\end{array}$} & $k-\varepsilon s t d$. & 3.7 & 9.6 & 10.5 \\
\hline & & RSM & 2.6 & 8.6 & 9.3 \\
\hline \multirow{6}{*}{$\begin{array}{c}C J \\
{\left[y_{c}, z_{c}\right]} \\
= \\
{[0,0]}\end{array}$} & $\begin{array}{l}\text { I) Paper I } \\
1 \times 6\end{array}$ & PIV & $4.5^{\mathrm{I}}$ & 22.2 & $>28.8^{\mathrm{I}}$ \\
\hline & \multirow{3}{*}{$\begin{array}{c}{ }^{\text {II)}} \text { Paper II } \\
6 \times 6, \text { planar data } z=0\end{array}$} & PIV & $6.6^{\mathrm{II})}$ & 14.9 & $21.1^{*}, 15^{\mathrm{II}), *}$ \\
\hline & & $k-\varepsilon$ std. & 5.0 & 13.1 & 17.3 \\
\hline & & RSM & 5.0 & 13.1 & 15.2 \\
\hline & \multirow{2}{*}{$\begin{array}{l}\text { Paper IV: } \\
6 \times 6 \text {, complete flow field }\end{array}$} & $k-\varepsilon$ std. & 4.9 & 13.4 & 21.7 \\
\hline & & $\mathrm{RSM}$ & 4.9 & 13.2 & 15.4 \\
\hline
\end{tabular}

* In Paper II $x_{c p} / d_{0}$ was approximated to $x_{c p} / d_{0} \approx 15$ based on the flat and uniform appearance of maximum velocity in different planes (see Figure 34). Small differences of stream-wise velocity around the $C J$ can however be detected up to $x / d_{0} \approx 21$.

** The values represent the first and second time merging criteria is reached, due to the local maxima in $U_{\text {min, } J} / U_{b}$ (see Figure 40)

For the $6 \times 6$ array the more rapid merging of the jets lead to values of $x_{c p} / d_{0}$ that for the $S J$ are similar to reported values in plane twin jet configurations. The $C J s$ in the $6 \times 6$ array combine later compared to twin plane jets but faster compared to twin or multiple round jet in a single row of jets. The PIV results at $z=0$ (Paper II) indicate that $x_{m p} / d_{0} \approx 6$ in the $6 \times 6$ array, which is further downstream compared to reported twin plane jet data. However, the CFD results in Paper IV show that the SJ merges asymmetrically with neighboring jets. Considering merging in all directions, the CFD simulation predicts that $x_{m p} / d_{0} \approx 3-4$ for the SJ. This is rather similar to twin plane jets having the same jet spacing. 


\subsection{Parametrical investigation}

This section reports on the parametrical investigations conducted in the present work. In Paper $\mathrm{V}$ the influence of five different independent factors (parameters) were investigated, using a combination of CFD simulations and Response Surface Methodology. Further investigation on the influence of dimensionless jet spacing and confluent jet array size was conducted in Paper VI, studying 4 out of the 41 CFD simulation setups (runs) in Paper V, where Case I to IV in Paper VI are equivalent to Run 13 to 16 in Paper V. In Paper V RS models of 13 different response variables were generated. Table 11 provides a list of investigated response variables, together with a brief description. More complete definitions of the response variables are found in Paper V.

Table 11 Summary of investigated response variables in Paper V.

\begin{tabular}{|c|c|c|}
\hline Flow feature & $\begin{array}{l}\text { Response } \\
\text { variables }\end{array}$ & Description \\
\hline $\begin{array}{l}\text { Confluent Core } \\
\text { Zone (CCZ) }\end{array}$ & $\begin{array}{c}x_{\text {start }} / d_{0} \\
x_{\text {end }} / d_{s} \\
U_{\text {core }} / U_{b}\end{array}$ & $\begin{array}{l}x_{\text {start }} / d_{0} \text { and } x_{\text {end }} / d_{s} \text { represents the start and } \\
\text { termination of the CCZ (see Figure } 45) \text { using nozzle } \\
\text { diameter }\left(d_{0}\right) \text { and effective source diameter }\left(d_{s}\right) \text { as } \\
\text { scaling length respectively. } \\
U_{\text {core }} / U_{b} \text { is the average of maximum stream-wise } \\
\text { velocity throughout the CCZ. }\end{array}$ \\
\hline $\begin{array}{l}\text { Turbulent flow field } \\
\quad \text { (turbulence } \\
\text { intensity) }\end{array}$ & $\begin{array}{l}\text { Peak value, } \\
T_{U, \min } \\
\text { Local minimum, } \\
T_{U, \min }\end{array}$ & $\begin{array}{l}\text { Local extreme values of minimum turbulence } \\
\text { intensity; peak value upstream of the CCZ and local } \\
\text { minimum at the end of the CCZ (see Figure 38) }\end{array}$ \\
\hline $\begin{array}{l}\text { Merging point } \\
\text { locations }\end{array}$ & $x_{m p} / d_{0}$ & $\begin{array}{l}\text { Locations of merging points, } x_{m p} / d_{0} \text {, reported for } \\
\text { three different jets, one } C J \text { next to the array center, } \\
\text { one } S J \text { next to the horizontal symmetry plane and } \\
\text { one } C o J \text {. For example on the placement of } \\
\text { investigated jets, please see Figure } 41 .\end{array}$ \\
\hline $\begin{array}{l}\text { Adjusted combined } \\
\text { point locations }\end{array}$ & $x_{a c p} / d_{0}$ & $\begin{array}{l}\text { Locations of adjusted combined points for the same } \\
\text { jets as described above. }\end{array}$ \\
\hline $\begin{array}{c}\text { Flow field } \\
\text { development }\end{array}$ & $\begin{array}{c}d\left(Q / Q_{0}\right) / d\left(x / d_{s}\right) \\
Q_{i} / Q_{0}\end{array}$ & $\begin{array}{l}d\left(Q / Q_{0}\right) / d\left(x / d_{s}\right) \text { represents the (constant) entrainment } \\
\text { rate of the combined jet.* } \\
Q_{i} / Q_{0} \text { is the } y \text {-axis interceptor of the linear } \\
\text { regression used to compute } d\left(Q / Q_{0}\right) / d\left(x / d_{s}\right) . Q_{i} / Q_{0} \text { is } \\
\text { a measure of the level of entrainment near the } \\
\text { nozzles, upstream of the combined region. }\end{array}$ \\
\hline
\end{tabular}

* computed between $x_{a c p} / d_{0}$ and a maximum downstream distance of $x=500 \mathrm{~mm}$ (42 to $\left.125 d_{0}\right)$. 
The PIV measurements in Paper I and II were performed at three Reynolds numbers, by changing the inlet bulk velocity $\left(U_{b}\right)$. For the $6 \times 6$ jet array the following two aspects of Reynolds number dependency was observed. The inwards bending of the jets increased with higher $U_{b}$ and the Reynolds stresses were found to increase more rapidly close to the nozzles when $U_{b}$ increased.

Inlet velocity is also a factor in Paper V. The RS models predict a statistically significant influence from $U_{b}$ on most response variables. However, the RS models show that for many of the response variables the influence from $U_{b}$ is relatively small, compared to factors related to jet spacing or the number of jets in the array. This is in good agreement with the PIV measurements in Paper II, where only limited Reynolds number dependency was observed.

The increase in inwards jet bending when $U_{b}$ increases, reported by the PIV measurements in Paper II, is however supported by the CFD simulations used to generate the RS models in Paper V. The same increase in inwards jet bending is observed when comparing CFD simulations of design points (runs) where an increase in $U_{b}$ is the only change of the included factors.

\subsubsection{Accuracy of RS models}

Even though the main objective of the RS models in Paper V is identifying principal influence of included factors it is still important to ensure that the resulting RS models can predict the results of the validated CFD model (Paper IV) with reasonable accuracy. By evaluating the ability of the RS models to predict correct values of the response variables in the validation case, the usability of the method in Paper $\mathrm{V}$ is addressed.

A comparison between the values of all response variables reported in Paper $\mathrm{V}$, extracted directly from the validation case CFD data (Paper IV), and predictions by the RS models are found in Table 12, together with the range of the response variables in the 41 runs used to generate the RS models. The $R^{2}$-values of the derived RS models are also added to Table 12, as a measure of how well the RS models approximate the design point data from CFD simulations.

The RS models generally predict the values of response variables in the validation case within a few percentages. The largest differences are reported for the CCZ velocity $\left(U_{\text {core }} / U_{b}\right)$ and $x_{m p} / d_{0}$ for the $S J$ which shows a difference of $10.1 \%$ and $16.5 \%$. The latter was rather expected as this point both lies close to nozzles and since the RS model had somewhat lower $\mathrm{R}^{2}$-value for this property. For $U_{\text {core }} / U_{b}$ the RS model has a high $R^{2}$-value, which indicates that the observed difference instead reflects that $U_{\text {core }} / U_{b}$ is more sensitive for inlet boundary conditions compared to other response variables. 
Table 12 Comparison between CFD results in the validation case (Paper IV) and RS model predictions in Paper V.

\begin{tabular}{|c|c|c|c|c|c|}
\hline Response variable & $\begin{array}{l}\text { CFD } \\
\text { results in } \\
\text { validation } \\
\text { case }\end{array}$ & $\begin{array}{l}\text { Predicted } \\
\text { values by } \\
\text { RS model }\end{array}$ & $\begin{array}{c}\text { Difference } \\
\text { between } \\
\text { CFD and } \\
\text { RS model }\end{array}$ & $\begin{array}{c}R^{2} \text {-value } \\
\text { of } \\
\text { RS model }\end{array}$ & $\begin{array}{c}\text { Range of values } \\
\text { in all RS model } \\
\text { design points } \\
\text { (Run } 1 \text { to } 41 \text { ) }\end{array}$ \\
\hline \multicolumn{6}{|c|}{ CCZ properties } \\
\hline$x_{\text {start }} / d_{0}$ & 20.2 & $21.4^{T}$ & $5.8 \%$ & $83.2 \%$ & $13.6-25.8$ \\
\hline$x_{\text {end }} / d_{s}$ & 10.7 & 11.2 & $4.7 \%$ & $98.6 \%$ & $8.6-15.9$ \\
\hline$U_{\text {core }} / U_{b}$ & 0.421 & 0.379 & $-10.1 \%$ & $99.9 \%$ & $0.273-0.605$ \\
\hline \multicolumn{6}{|c|}{ Minimum turbulence intensity, $T_{U, \text { min }}$} \\
\hline Peak value, $T_{U, \min }$ & 0.248 & 0.242 & $-2.3 \%$ & $99.7 \%$ & $0.132-0.302$ \\
\hline Local minimum $T_{U \text { min }}$ & 0.0805 & 0.812 & $0.8 \%$ & $99.8 \%$ & $0.043-0.082$ \\
\hline \multicolumn{6}{|c|}{ Merging point locations, $x_{m p} / d_{0}$, for different jets } \\
\hline$C J$ & 4.9 & 4.7 & $-4.8 \%$ & $99.3 \%$ & $0.9-8.5$ \\
\hline SJ & 3.7 & 3.1 & $-16.5 \%$ & $89.2 \%$ & $0.2-6.2$ \\
\hline $\mathrm{CoJ}$ & 4.4 & 4.2 & $-4.0 \%$ & $99.1 \%$ & $1.0-6.9$ \\
\hline \multicolumn{6}{|c|}{ Adjusted combined point locations, $x_{a c p} / d_{0}$} \\
\hline$C J$ & 13.4 & 14.0 & $3.9 \%$ & $99.1 \%$ & $8.8-17.0$ \\
\hline SJ & 9.5 & 9.2 & $-3.1 \%$ & $98.4 \%$ & $5.2-10.7$ \\
\hline CoJ & 11.1 & 11.6 & $5.0 \%$ & $99.1 \%$ & $7.4-14.5$ \\
\hline \multicolumn{6}{|c|}{ Volumetric flow rate of the combined jet } \\
\hline$Q_{i} / Q_{0}$ & 2.098 & 2.046 & $-2.5 \%$ & $99.5 \%$ & $1.217-2.759$ \\
\hline$d\left(Q / Q_{0}\right) / d\left(x / d_{s}\right)$ & 0.250 & 0.232 & $-7.1 \%$ & $99.3 \%$ & $0.181-0.255$ \\
\hline
\end{tabular}

\subsubsection{Merging and combining characteristics}

Nozzle height $(b)$ has either small or no significant influence on the response variables and the influence of $h$ is therefore only referred to in Paper V, and not discussed in this section. Dimensionless nozzle spacing, $S / d_{0}$, has a large influence on most parameters. Figure 41 shows $x_{m p} / d_{0}$ and $x_{a c p} / d_{0}$ plotted against $S / d_{0}$ for the three investigated jets. Increasing $S / d_{0}$ extends the merging and adjusted combined point distance for all investigated jets. The location of $x_{m p} / d_{0}$ and $x_{a c p} / d_{0}$ also depend on jet position in the array. SJs interact more rapidly with neighboring jets than do CoJs and $C J \mathrm{~s}$, leading to lower values of $x_{m p} / d_{0}$ and $x_{a c p} / d_{0}$ for the $S J$. The number of jets in the array is also an important parameter influencing $x_{a c p} / d_{0}$, where larger jet arrays sizes reduce $x_{a c p} / d_{0}$ for all investigated jets.

For twin plane jets sub-atmospheric pressure between jets plays an important role in jet merging, acting to draw the jets together and combine with each other. In Paper VI the connection between static pressure, jet interactions, lateral jet displacement and adjusted combined point distance was investigated. Figure 42 shows iso-contours of the static pressure having values of $-2 \mathrm{~Pa}$ and $-8 \mathrm{~Pa}$, relative to the CFD model operating pressure of $101.3 \mathrm{kPa}$, in the four setups investigated in Paper VI. In all setups sub-atmospheric pressure develops close to the jet inlets. The strongest subatmospheric pressure is associated with the $S J$ s located at $z_{c}=0$ and $y_{c}=0 . C J \mathrm{~s}$ and 
CoJs, which have larger values of $x_{a c p} / d_{0}$ (see Figure 41) experience weaker subpressure.

In Table 13 numerical values of the minimum static pressure between jets in the array is presented together with $x_{a c p} / d_{0}$ for the $S J$ located at $z_{c}=0$ in the four setups found in Figure 42. The table shows the connection between stronger sub-pressure and lower $x_{a c p} / d_{0}$ for the $S J$, where either smaller jet spacing or larger array size increase the strength of the sub-pressure and reduce $x_{a c p} / d_{0}$. The results in Paper IV also showed that stronger sub-pressure resulted in stronger jet deformation of the SJ. In summary it is concluded that sub-pressure between jets is strongly connected to the jet interactions, also in confluent round jet setups. As a result, stronger sub-pressure results in shorter adjusted combined point distances and stronger jet deformation.
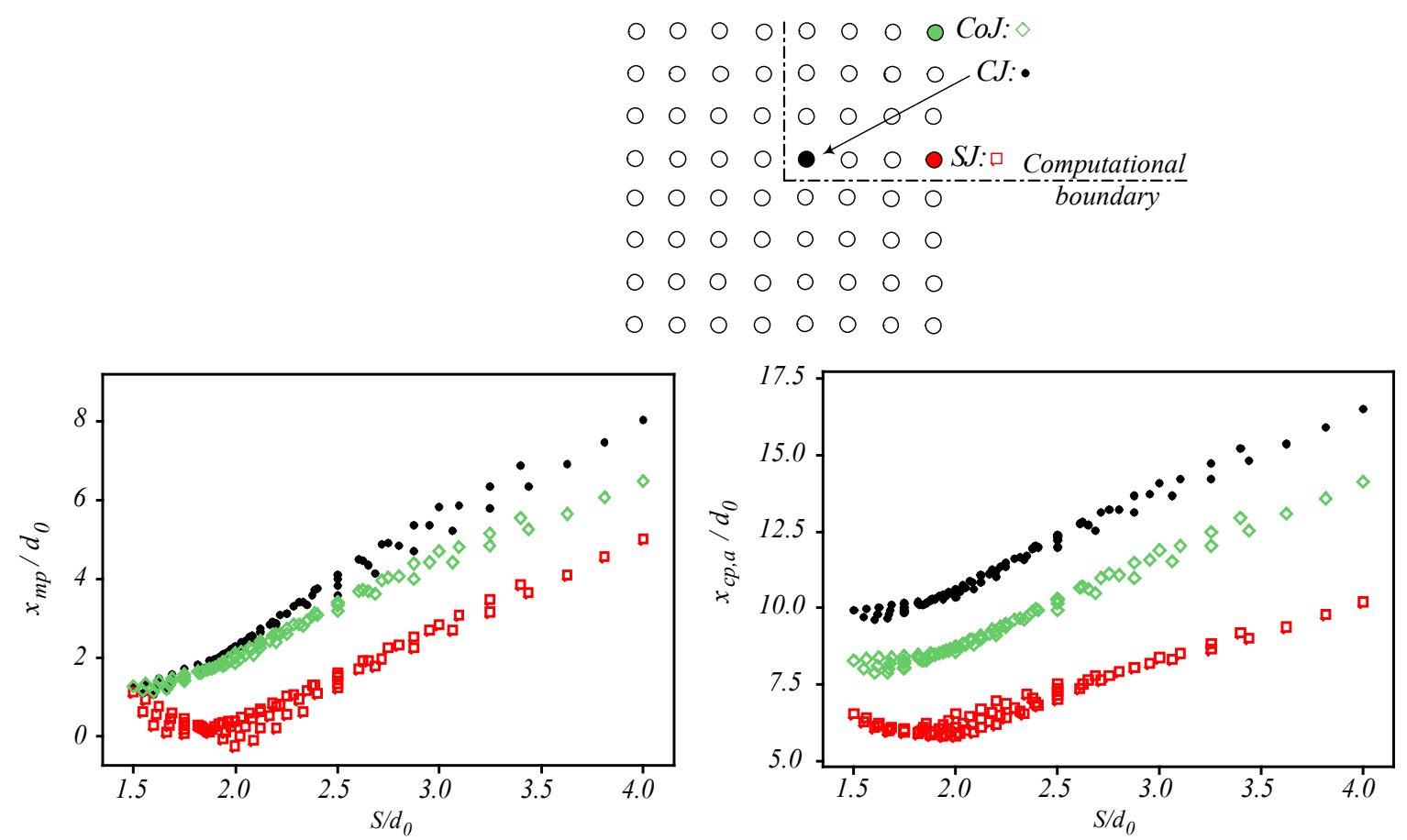

Figure 41 RS model response for $x_{m p} / d_{0}$ (left) and $x_{a c p} / d_{0}$ (right) for three different jets plotted against $S / d_{0} . n, U_{b}$ and $h$ are held fixed at the medium level.

Table 13 Relationship between array size, jet spacing, static pressure and adjusted combined point of the $S J$ at $z_{c}=0$.

\begin{tabular}{ccccc}
\hline $\begin{array}{c}\text { Design point } \\
\text { in Paper V } \\
\text { (Run) }\end{array}$ & $\begin{array}{c}\text { Dimensionless } \\
\text { jet spacing } \\
\text { S/d }\end{array}$ & $\begin{array}{c}\text { Number of jets } \\
\text { per row } \\
(n)\end{array}$ & $\begin{array}{c}\text { Minimum pressure } \\
\text { between jets } \\
(\mathrm{Pa})\end{array}$ & $x_{\text {acp }} / d_{0}$ \\
\hline 13 & 3.25 & 6 & -2.7 & 10.7 \\
14 & 1.75 & 6 & -9.8 & 6.8 \\
15 & 3.25 & 10 & -6.7 & 7.4 \\
16 & 1.75 & 10 & -17.6 & 5.4 \\
\hline
\end{tabular}



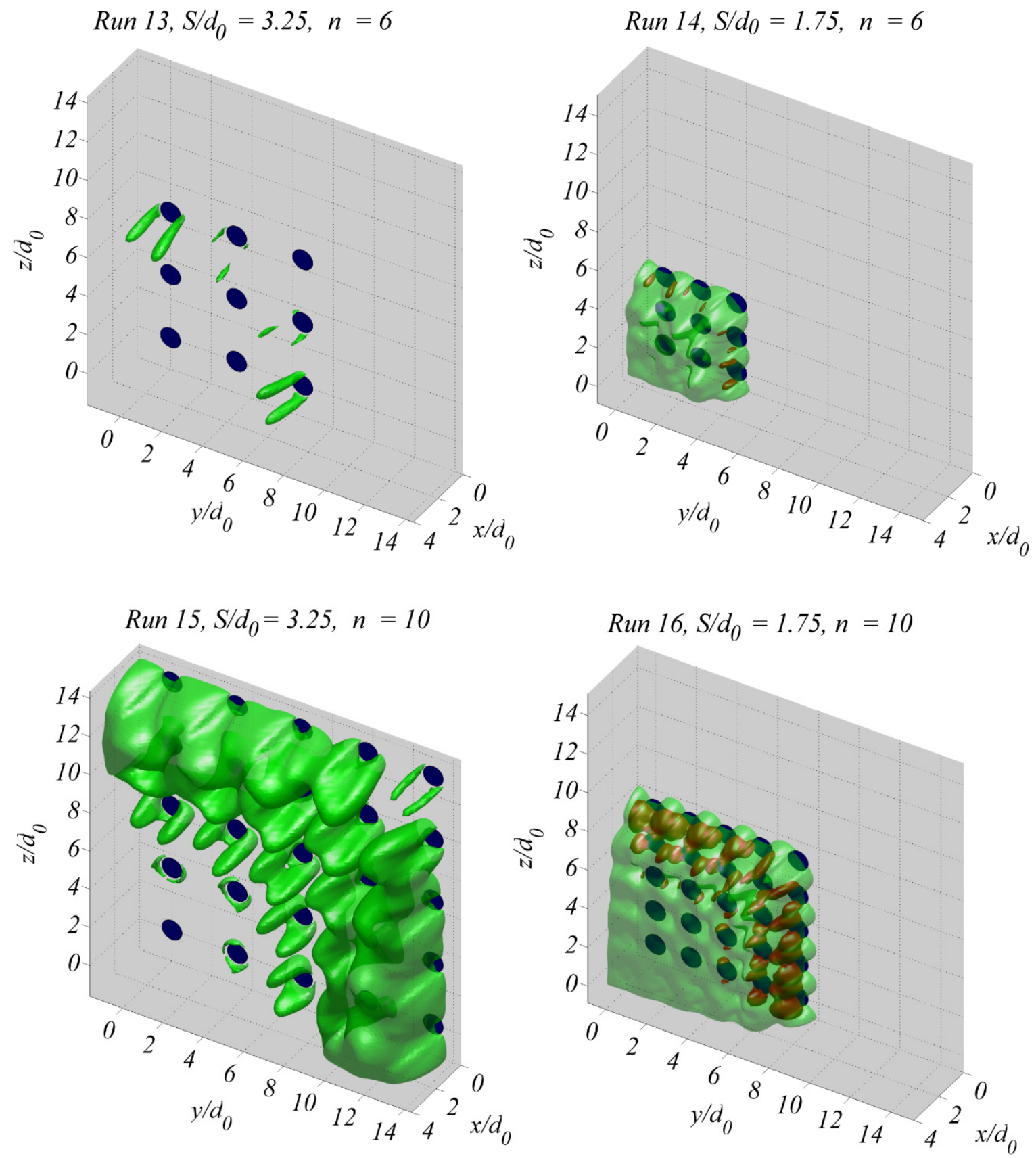

Figure 42 Iso-contours of static sub-pressure close to the jet inlets. Semitransparent green represents $-2 \mathrm{~Pa}$ and red $-8 \mathrm{~Pa}$, relative to CFD model operating pressure $(101.3 \mathrm{kPa})$.

In Section 8.6.1 it was concluded that accurate RS model predictions of $x_{m p} / d_{0}$ were difficult for the SJ. In Paper VI it was concluded that there are two different flow structures on the inside of the SJ. Depending on the jet spacing, the recirculation behind the SJ consists of either a single vortex or a Counter-Rotating Cortices Pair (CVP). Examples of the two different flow structures are shown in Figure 43. The switch-over effect explains the problems in predicting $x_{m p} / d_{0}$ by a quadratic RS model. The existence of either a single vortex or a CVP also has implications for the jet deformation, a process which is described in Paper VI. 

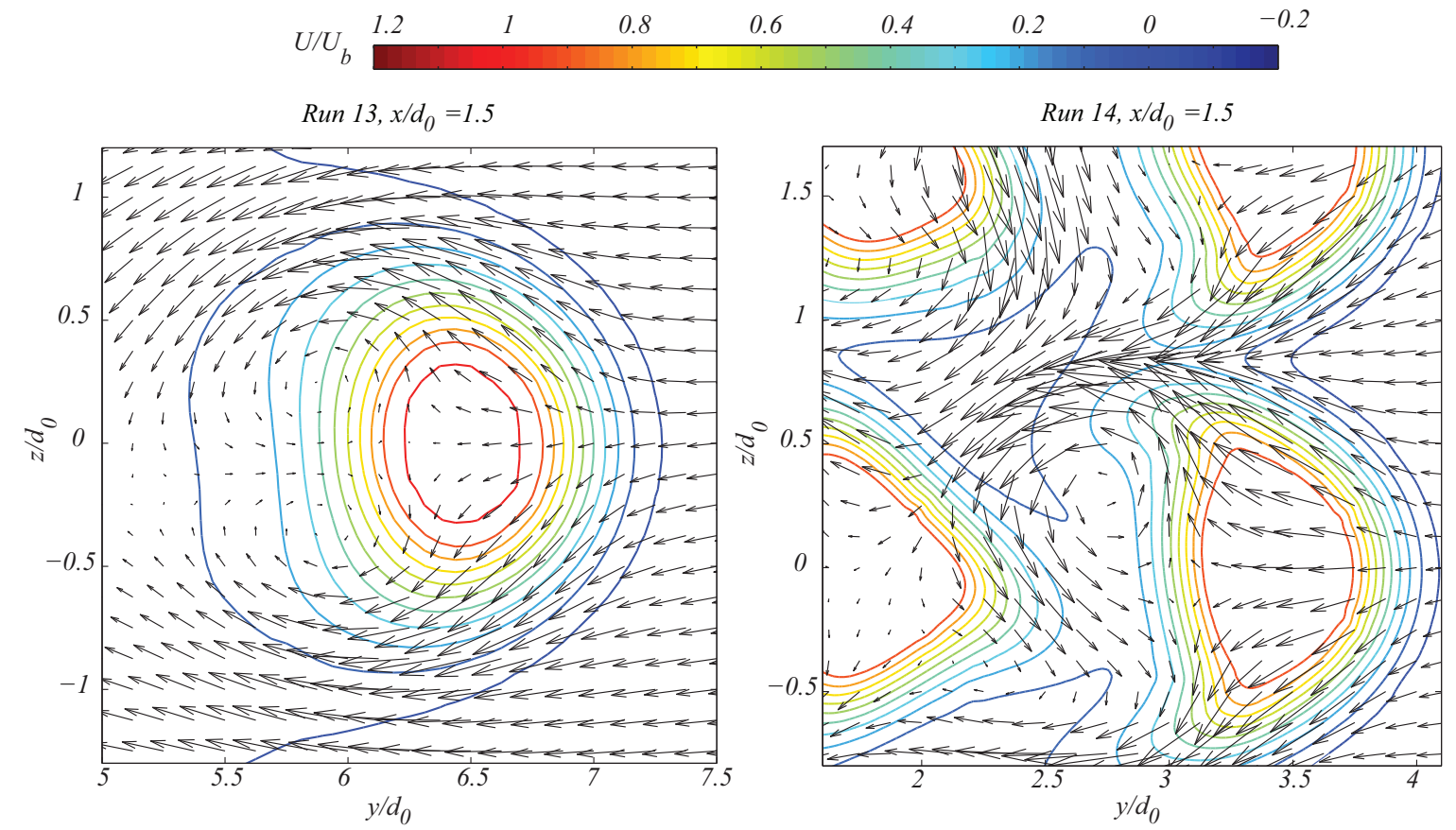

Figure 43 Contours of $U / U_{b}$ for the $S J$ located at $z_{c}=0$ overlaid with vector field of lateral velocity components in Run 13 and 14.

\subsubsection{Turbulence intensity}

The development of minimum turbulence intensity of the confluent jets, $T_{U \text {,min }}$, has two local extreme points in all runs. In the merging region a maximum in $T_{U, \min }$ occurs. $T_{U, \min }$ will then decrease and reach a local minimum at the end of the CCZ. In Figure 44 the values of the two local extreme points in $T_{U, \min }$, predicted by the RS models, are plotted against $S / d_{0}$.
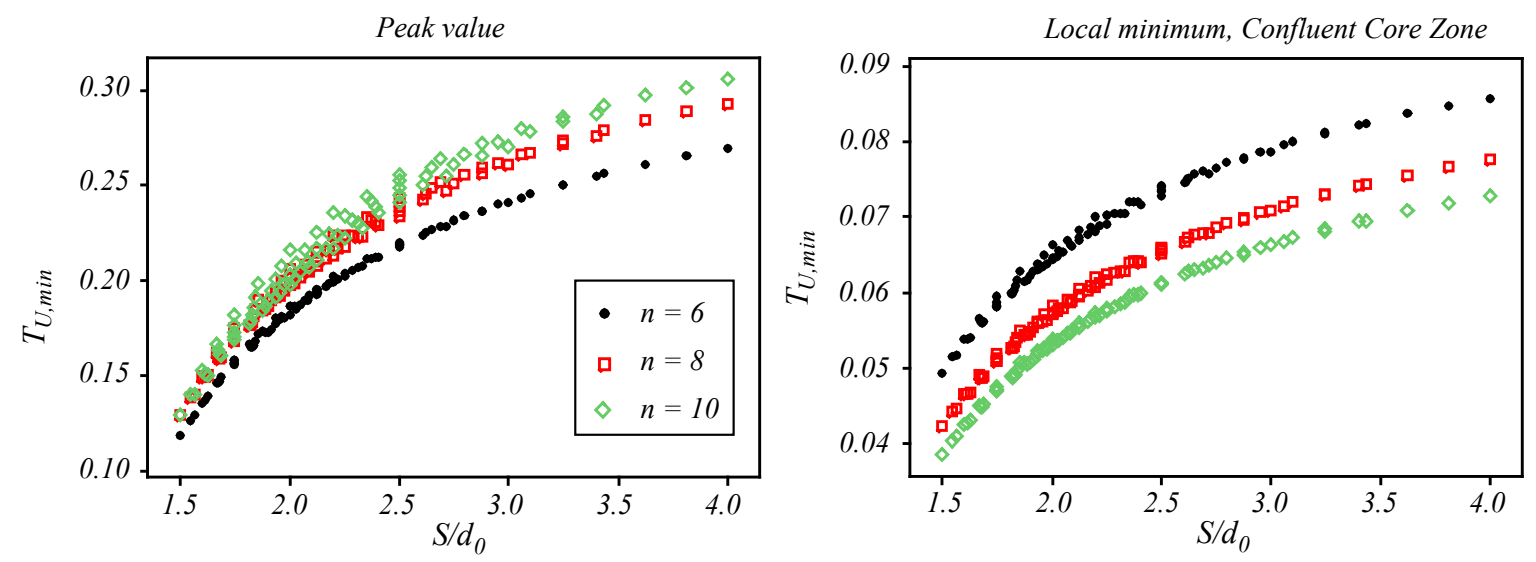

Figure $44 \mathrm{RS}$ response for the peak value and local minimum of $T_{U, \text { min }}$ using different combinations of $n, E$, and $d_{0} . U_{b}$ and $h$ are fixed at the medium level.

For small $S / d_{0}$, the maximum value of $T_{U, \min }$ only reach approximately $12 \%$. This is lower compared to the experimental study on a single round jet by Todde et al. (2009) where stream-wise turbulence intensity at the jet centerline reached peak values between 21 and $28 \%$. 
In the CCZ $T_{U, \min }$ reduces to less than $10 \%$ in all runs. A larger number of jets per row $(n)$ increases the peak value but reduces the minimum in the CCZ (see Figure 44). The latter is mainly due to a longer CCZ, which leads to a longer region with decay of $T_{U, \text { min }}$.

\subsubsection{Confluent Core Zone and maximum velocity decay}

The maximum velocity decays linearly, in accordance with Eq. (1), both upstream of the CCZ, in the merging region, and downstream of the CCZ. Results in Paper V showed that within the CCZ the appropriate scaling length changes from the inlet diameter of a single nozzle $\left(d_{0}\right)$ to the effective source diameter $\left(d_{s}\right)$ for the array. The effective source diameter is calculated according to Eq. (76) in the investigated square array of round jets.

$d_{s}=\sqrt{4 A_{\text {inlet }} / \pi}=\sqrt{4 \cdot n^{2}\left(\frac{\pi}{4} d_{0}^{2}\right) / \pi}=n \cdot d_{0}$

where $n$ is the number of jets per row.

If $d_{0}$ and $d_{s}$ are used as scaling length upstream and downstream of the CCZ, the velocity decay coefficient in Eq. (1) attains values in the same order of size as a single round jet. An example of this is illustrated in Figure 45 where the decay of maximum velocity in Run 3 and 13, having different values of both $d_{0}$ and $d_{s}$, is presented.
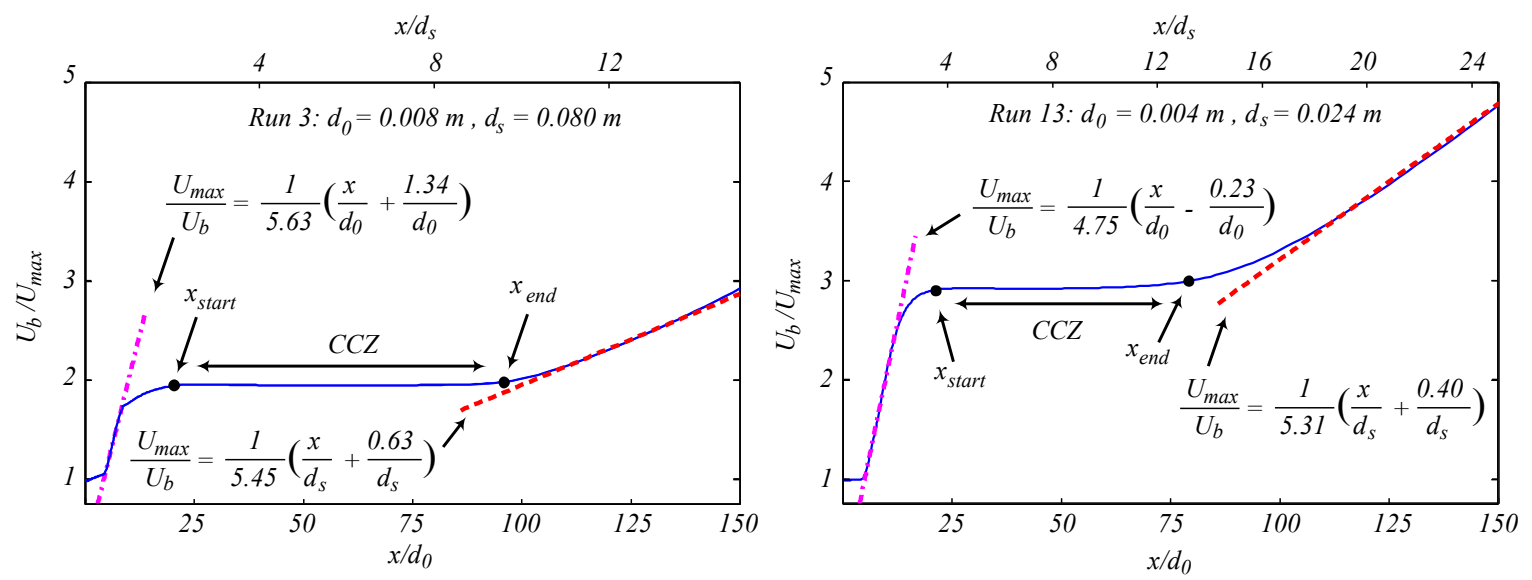

Figure 45 Development of $U_{b} / U_{\max }$ and locations of $x_{\text {start }}$ and $x_{\text {end }}$ for Run 3 (left) and Run 13 (right).

Larger $S / d_{0}$ reduces the velocity in the CCZ $\left(U_{\text {core }} / U_{b}\right)$, due to higher jet expansion ratio. Increasing $S / d_{0}$ also extends the $C C Z$ further downstream due to a larger crosssectional area of the CCZ, which delays the penetration of turbulent shear layers to the combined jet center. Larger arrays extend the CCZ in the downstream direction, even when the downstream extent of the CCZ is expressed in effective source diameters. Larger arrays also reduce $U_{c o r e} / U_{b}$. In Paper VI this was attributed to the fact that larger arrays have less entrainment of surrounding fluid between jets to the array center close to the inlets. As the volumetric flow rate in the center part of the array is lower when the $C J s$ combine and form a zone of uniform velocity, $U_{\text {core }} / U_{b}$ attains a lower value in larger arrays. A higher inlet velocity, $U_{b}$, will move the CCZ downstream, as both $x_{\text {start }} / d_{0}$ and $x_{\text {end }} / d_{s}$ increases with higher $U_{b}$. 


\subsubsection{Volumetric flow rate}

The development of volumetric flow rate of the confluent jets, $Q / Q_{0}$, calculated according to Eq. (77), can be divided in two regions, one upstream of the combined point with high entrainment and a decreasing entrainment rate and one region with a constant entrainment rate as the jets are combined.

$$
\frac{Q}{Q_{0}}=\frac{1}{n^{2} \cdot U_{b} \cdot \frac{\pi}{4} d_{0}^{2}} \cdot \iint_{U \geq 0} U d y d z
$$

An example of the development of volumetric flow rate, and the meaning of the two quantities $d\left(Q / Q_{0}\right) / d\left(x / d_{s}\right)$ and $Q_{i} / Q_{0}$, for which RS models were generated is presented in Figure 46. It can be noted that the investigation of volumetric flow rate was limited to $x=500 \mathrm{~mm}$ (42 to $125 d_{0}$ ) in Paper $\mathrm{V}$ in order to avoid influence from far field boundaries.

Within the investigated downstream region the entrainment rate of the combined jet, $d\left(Q / Q_{0}\right) / d\left(x / d_{s}\right)$, is lower than that of a fully developed single round jet. However, the entrainment next to the nozzles, represented by $Q_{i} / Q_{0}$, has a large influence on the flow rate to the combined jet. Interestingly $d\left(Q / Q_{0}\right) / d\left(x / d_{s}\right)$, does not correlate with $S / d_{0}$. Instead all investigated parameters that increase the cross-sectional area of the nozzle plate, such as edge-to-edge nozzle spacing, nozzle diameter and number of jets per row, reduce the entrainment rate of the combined jet. Reducing $S / d_{0}$ reduces the entrainment near the nozzles. Larger matrices also decrease the entrainment of ambient air near the nozzles, relative to the inlet flow rate $Q_{0}$.

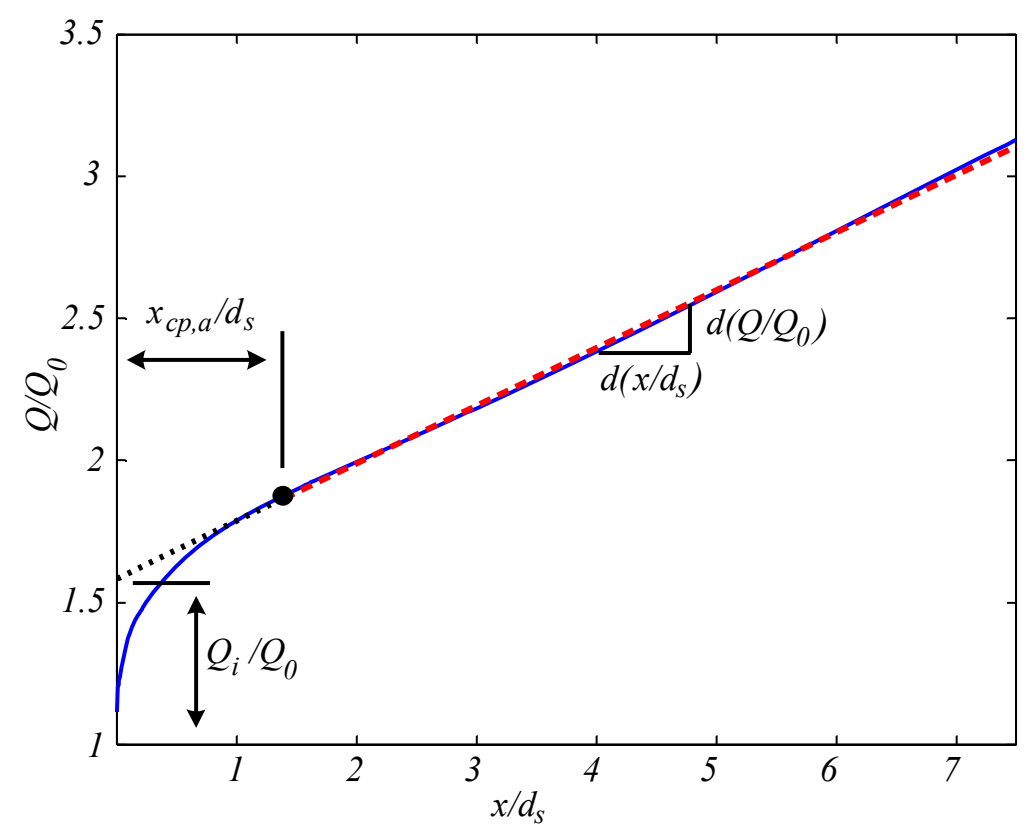

Figure 46 Development of volumetric flow rate in Run 41. Solid line: $Q / Q_{0}$. Dashed line: linear regression downstream of adjusted combined point for the $C J$ at the array center. Dotted line: extrapolation of linear regression 


\section{Conclusions}

This section summarizes the findings of the present work in relation to the posed research questions and aim of the study.

The aim of the present work was to use advanced measurement techniques and CFD simulations to provide deeper understanding of the turbulent flow field development of unconfined confluent round jets. By using LDA and PIV measurements, CFD simulations and statistical methods, the present work has identified and quantified several important aspects related to the flow field development within the investigated unconfined confluent jet arrays.

The employed RANS turbulence models, i.e., the $k-\varepsilon$ std., RNG $k-\varepsilon$ and RSM models, were all capable of accurately predicting mean velocities and turbulent properties in the investigated confluent jet array. In general the RSM and $k-\varepsilon s t d$. models provided smaller deviations between numerical and experimental results than the RNG $k-\varepsilon$ model. In terms of mean velocity the second-order closure model (RSM) was not found to be superior to the less complex $k-\varepsilon$ std. model. The RSM provides satisfactory agreement of stream-wise normal Reynolds stress $\left(\overline{u^{\prime 2}}\right)$ and shear stress $\left(\overline{u^{\prime} v^{\prime}}\right)$, but generally underpredicts the normal Reynolds stress in the span-wise direction $\left(\overline{v^{\prime 2}}\right)$. All models could predict the development of turbulent kinetic energy $(k)$, in locations where array symmetry could be used to approximate $k$ in the experimental data. Results with the $k-\varepsilon s t d$. also showed that stream-wise turbulence intensity could be predicted with satisfactory agreement compared with PIV measurements, as long as the velocity in the stream-wise direction $(U)$ was not too low.

The positions of the jets within the array strongly influence both mean velocity and turbulence of the jet. The jets may be divided in categories based on their position in the array. Square in-line jet arrays include three jet categories: Central Jets $(C J \mathrm{~s})$, Side Jets $(S J \mathrm{~s})$ and Corner Jets $(C o J)$. A single row of jets may be divided in Side Jets and Central Jets.

In both the investigated single row of $(1 \times 6)$ jets and square $(6 \times 6,8 \times 8$ or $10 \times 10)$ jet arrays all jets experience merging and combining. Square arrays also include considerable jet convergence, which was not present in the $1 \times 6$ array. Due to the jet convergence in square arrays the turbulent flow field, especially for jets far away from 
the array center, is affected by mean flow curvature. The mean flow curvature causes elevated level of turbulent kinetic energy in shear layers with concave curvature, i.e., facing away from the array center.

The jet convergence accelerates both merging and combining of the jets. The SJs in square arrays both merge and combine with neighboring jets faster than other jet categories. The strong jet-to-jet interactions for $S J \mathrm{~s}$ in square arrays result in considerable jet deformation, which in turn lead to shorter potential core, higher turbulent kinetic energy and faster velocity decay compared to other jets. The CoJ does not interact as strongly with neighboring jets as do the SJs. Therefore the CoJs are more similar to $C J s$ than $S J s$ in the investigated square jet arrays.

As the jets combine a zone with uniform stream-wise velocity and low turbulence intensity forms in the center of square jet arrays. This zone has been called Confluent Core Zone (CCZ) due to its similarities with the potential core zone of a single jet. Within the CCZ the appropriate scaling length changes from nozzle diameter to the effective source diameter. During the prevalence of the CCZ the combined jet experiences axis-switching between horizontal and diagonal directions, before adapting an axisymmetric shape.

In the $1 \times 6$ array the decay of maximum velocity of the $S J \mathrm{~s}$ is similar to a single round jet, where $1 / U_{\text {max }, J} \propto x$. The $C J$ s develop as $1 / U_{\text {max }, J} \propto \sqrt{x}$, similar to a plane jet, even when they are not effectively two-dimensional. Due to the CCZ the flow field development of square confluent jet arrays differs from both single round and plane jets, as well as round or plane twin jets, and a single row of round jets. None of the above unconfined jet configurations has any larger region with uniform velocity downstream of the potential core.

In twin or multiple jet configurations locations of merging and combined points are important characteristics, as also in the investigated confluent jet arrays. The combined point definition used in twin plane jets was considered too sensitive to small velocity differences, due to the creation of the CCZ, to provide a robust criterion for $C J \mathrm{~s}$ being combined. The introduction of an adjusted combined point provides a more reliable estimate of combined distance in unconfined confluent round jet arrays.

In square arrays the combined point locations for $S J$ s are comparable to twin plane jets at similar dimensionless jet spacing $\left(S / d_{0}\right)$ or $(S / w)$. CJs and $C o J$ s combine later than twin plane jets but faster than twin round jets or a single row of round jets. Contrary to twin plane jets there is no strong recirculation zone between jets in the present investigations. While backflow is occurring in some instances, especially between $S J s$, initiation to jet merging in square arrays rather has a complex threedimensional character and different flow structures develop on the inside of the SJs depending on the dimensionless jet spacing, $S / d_{0}$. Sub-atmospheric pressure develops between jets close to the nozzles and acts to deform the jets and draw them closer to the array center. SJs experience the strongest sub-atmospheric pressure. 
The parametrical investigation, using CFD simulations and Response Surface Methodology, showed that nozzle diameter, $d_{0}$, and edge-to-edge nozzle spacing, $E$, were the most important of the investigated parameters. This reflects a strong dependence on dimensionless jet spacing, $S / d_{0}$. Higher $S / d_{0}$ leads to a downstream shift of merging and adjusted combined points of all jets and a CCZ with lower velocity and longer downstream extension. Increasing $S / d_{0}$ also leads to lower turbulence intensity in the downstream extent of the CCZ. The entrainment rate of the combined jet, on the other hand, does not correlate with $S / d_{0}$.

Increasing the array size leads to a reduced adjusted combined point distance, as a stronger sub-atmospheric pressure between the jets causes the confluent jets to converge more strongly together. Larger array size also extends the CCZ, reduces the minimum turbulence intensity in the $C C Z$ and reduces the entrainment near the nozzles, relative to the inlet flow rate. A higher inlet velocity was found to increase the jet convergence in the investigated square confluent jet arrays. A higher inlet velocity also tends to move the CCZ in the downstream direction. Nozzle height generally has a small impact on the investigated response variables. 



\section{Future work 1}

This section discusses possible future studies on unconfined multiple interacting confluent jet arrays based on the outcome, and limitations, of the present work.

While the present work has provided a detailed investigation of multiple interacting confluent jet arrays, there are several aspects that remain to be investigated. The present investigations have only been conducted on in-line jet arrays, but there are several other ways in which confluent jet arrays may be arranged. Future investigations should therefore include hexagonal, staggered and circular arranged jet arrays. The parametrical investigation in the present work is also limited to only five parameters and needs to be expanded by considering for example different velocity ratio between jets and jet arrays having jets of varying diameters or temperature, just to mention a few examples.

Another topic for future studies is the influence of inlet boundary conditions. Common inlet boundary conditions include orifice jets, bell-shaped nozzles or jets exiting from long circular pipes. The possible impact of inlet boundary conditions, similar to the investigations on a single round jet by for example Quinn (2006) and Mi et al. (2001), should also be performed for confluent jet arrays. Future studies may also include ventilated confluent jet arrays as the present investigations are limited to short nozzles attached to a solid wall.

The current investigation is limited to a maximum downstream distance of $150 \mathrm{~d} 0$. While this includes the region with constant maximum velocity in all setups, far field behavior of the combined jet remains to be investigated. An interesting object of study is to compare the far field behavior of a combined confluent jet with single jet configurations.

The present work has only considered time averaged properties. Each PIV recording session however includes approximately 4,000 realizations of the instantaneous velocity field, which can be used to identify flow structures in the confluent jet arrays. Shown by for example Geers et al. (2005), Wang et al. (2007) and Wang et al. (2010) analysis of the second invariant of the velocity gradient tensor, kinematic vorticity number, probability density functions, two-point correlations, topology-based visualization, Galilean decomposition and Proper Orthogonal Decomposition (POD) are all means that can be used investigate flow structure behavior, based on PIV data. 
Information on flow structures would improve the understanding of the jet-to-jet interactions taking place in unconfined confluent jet arrays.

Further understanding of the development of flow structures and jet-to-jet interactions may also be acquired using time-resolved numerical simulations, such as Large Eddy Simulations (LES) or Scale Adaptive Simulations (SAS), or by conducting time-resolved PIV measurement. The experimental data in the present study may also be used for validation of future numerical simulations. 


\section{References}

Abdel-Rahman, A. 2010. A review of effects of initial and boundary conditions on turbulent jets. WSEAS Transactions on Fluid Mechanics, 5, 257-275.

Absil, L. H. J. 1995. Analysis of the Laser Doppler Measurement Technique for Application in Turbulent Flows, Delft University of Technology, Faculty of Aerospace Engineering.

Adrian, R. J. \& Westerweel, J. 2010. Particle Image Velocimetry, Cambridge University Press.

Albrecht, H.-E. 2003. Laser doppler and phase doppler measurement techniques, Berlin ; New York, Springer.

Allen, J. B. \& Smith, D. L. 2011. Numerical modeling of turbulent, parallel, round jets. 37.

Alvarez, L. F. 2000. Design optimization based on genetic programming : approximation model building for design optimization using the response surface methodology and genetic programming. PhD Thesis, University of Bradford.

Anderson, E. A. \& Spall, R. E. 2001. Experimental and numerical investigation of twodimensional parallel jets. Journal of Fluids Engineering, Transactions of the ASME, 123, 401-406.

Anderson, J. 1995. Computational Fluid Dynamics, McGraw-Hill Education.

ANSYS Inc. 2012. ANSYS FLUENT User's Guide, Release 14.5. Canonsburg.

Awbi, H. B. 2003. Ventilation of Buildings, London, Spon Press.

Ball, C. G., Fellouah, H. \& Pollard, A. 2012. The flow field in turbulent round free jets. Progress in Aerospace Sciences, 50, 1-26.

Baratian-Ghorghi, Z., Kaye, N. B., Khan, A. A. \& Smith, J. R. 2012. The merging of two unequal axisymmetric parallel turbulent jets. Journal of Hydrodynamics, 24, 257262.

Boersma, B. J., Brethouwer, G. \& Nieuwstadt, F. T. M. 1998. A numerical investigation on the effect of the inflow conditions on the self-similar region of a round jet. Physics of Fluids, 10, 899-909.

Boutazakhti, M., Sullivan, P. E., Thomson, M. J. \& Yimer, I. 2007. Flow downstream of a cluster of nine jets. Journal of Fluids Engineering, Transactions of the ASME, $129,541-550$.

Boutier, A. 2013. Laser Velocimetry in Fluid Mechanics, Wiley.

Box, G. E. \& Draper, N. R. 1987. Empirical model-building and response surfaces, John Wiley \& Sons.

Box, G. E. P. \& Wilson, K. B. 1951. On the Experimental Attainment of Optimum Conditions. Journal of the Royal Statistical Society. Series B (Methodological), $13,1-45$.

Box, G. E. P. \& Behnken, D. W. 1960. Some New Three Level Designs for the Study of Quantitative Variables. Technometrics, 2, 455-475.

Bradshaw, P. 1973. Effects of streamline curvature on turbulent flow. AGARDograph $A G-169$. 


\section{Papers}

The articles associated with this thesis have been removed for copyright reasons. For more details about these see:

http://urn.kb.se/resolve?urn=urn:nbn:se:liu:diva-117066 
Böhm, B., Stein, O., Kempf, A. \& Dreizler, A. 2010. In-nozzle measurements of a turbulent opposed jet using PIV. Flow, Turbulence and Combustion, 85, 73-93.

Chen, H., Moshfegh, B. \& Cehlin, M. 2013. Computational investigation on the factors influencing thermal comfort for impinging jet ventilation. Building and Environment, 66, 29-41.

Chen, Q. 1995. Comparison of different k- $\varepsilon$ models for indoor air flow computations. Numerical Heat Transfer, Part B: Fundamentals, 28, 353-369.

Chen, Q. 2009. Ventilation performance prediction for buildings: A method overview and recent applications. Building and Environment, 44, 848-858.

Corrsin, S. 1944. Investigation of the behavior of parallel two-dimensional air jets. NASA, No. $4 \mathrm{H} 24$.

Crow, S. C. \& Champagne, F. H. 1971. Orderly structure in jet turbulence. Journal of Fluid Mechanics, 48, 547-591.

Durve, A., Patwardhan, A. W., Banarjee, I., Padmakumar, G. \& Vaidyanathan, G. 2012. Numerical investigation of mixing in parallel jets. Nuclear Engineering and Design, 242, 78-90.

Fellouah, H., Ball, C. G. \& Pollard, A. 2009. Reynolds number effects within the development region of a turbulent round free jet. International Journal of Heat and Mass Transfer, 52, 3943-3954.

Ferreira, S. L. C., Bruns, R. E., Ferreira, H. S., Matos, G. D., David, J. M., Brandão, G. C., da Silva, E. G. P., Portugal, L. A., dos Reis, P. S., Souza, A. S. \& dos Santos, W. N. L. 2007. Box-Behnken design: An alternative for the optimization of analytical methods. Analytica Chimica Acta, 597, 179-186.

Foss, J. F., Tropea, C. \& Yarin, A. L. 2007. Springer Handbook of Experimental Fluid Mechanics, Springer Verlag.

Freitas, C. J. 1993. Editorial. Journal of Fluids Engineering, 115, 339-340.

Fujisawa, N., Nakamura, K. \& Srinivas, K. 2004. Interaction of two parallel plane jets of different velocities. Journal of Visualization, 7, 135-142.

Geers, L. F. G. 2004. Multiple impinging jet arrays: an experimental study on flow and heat transfer. PhD Thesis, Delft University of Technology.

Geers, L. F. G., Tummers, M. J. \& Hanjalić, K. 2005. Particle imaging velocimetry-based identification of coherent structures in normally impinging multiple jets. Physics of Fluids, 17, 1-13.

Geers, L. F. G., Hanjalic, K. \& Tummers, M. J. 2006. Wall imprint of turbulent structures and heat transfer in multiple impinging jet arrays. Journal of Fluid Mechanics, 546, 255-284.

Ghahremanian, S. \& Moshfegh, B. 2014. Evaluation of RANS models in predicting low Reynolds, free, turbulent round jet. Journal of Fluids Engineering, Transactions of the ASME, 136.

Gibson, M. M. \& Launder, B. E. 1978. Ground effects on pressure fluctuations in the atmospheric boundary layer. Journal of Fluid Mechanics, 86, 491-511.

Goldstein, R. 1996. Fluid Mechanics Measurements, Second Edition, Taylor \& Francis.

Grinstein, F. F., Gutmark, E. \& Parr, T. 1995. Near field dynamics of subsonic free square jets. A computational and experimental study. Physics of Fluids, 7, 14831497.

Grotjans, H. \& Menter, F. 1998. Wall functions for general application \{CFD $\}$ codes. In: Papailou, ed. ECCOMAS 98. 1112-1117.

Han, D. \& Mungal, M. G. 2001. Direct measurement of entrainment in reacting/nonreacting turbulent jets. Combustion and Flame, 124, 370-386. 
Hanjalić, K. 1999. Second-Moment Turbulence Closures for CFD: Needs and Prospects. International Journal of Computational Fluid Dynamics, 12, 67-97.

Harima, T., Fujita, S. \& Osaka, H. 2005. Turbulent properties of twin circular free jets with various nozzle spacing. Engineering Turbulence Modelling and Experiments $6,501-510$.

Harima, T. F., S.; Osaka, H. 2001. Mixing and Diffusion Processes of Twin Circular Free Jets with Various Nozzle Spacing. Experimental Heat Transfer Fluids Mechanics, and Thermodynamics 2001.

Hill, B. J. 1972. Measurement of local entrainment rate in the initial region of axisymmetric turbulent air jets. Journal of Fluid Mechanics, 51, 773-779.

Hussein, H. J., Capp, S. P. \& George, W. K. 1994. Velocity measurements in a highReynolds-number, momentum-conserving, axisymmetric, turbulent jet. Journal of Fluid Mechanics, 258, 31-75.

Janbakhsh, S., Moshfegh, B. \& Ghahremanian, S. 2010. A newly designed supply diffuser for industrial premises. International Journal of Ventilation, 9, 59-67.

Janbakhsh, S. \& Moshfegh, B. 2014. Experimental investigation of a ventilation system based on wall confluent jets. Building and Environment, 80, 18-31.

Khuri, A. I. \& Cornell, J. A. 1996. Response Surfaces: Designs and Analyses: Second Edition, Taylor \& Francis.

Knystautas, R. 1962. The turbulent jet from a series of holes in line, MERL Report 62-1. McGill University.

Ko, N. W. M. \& Lau, K. K. 1989. Flow structures in initial region of two interacting parallel plane jets. Experimental Thermal and Fluid Science, 2, 431-449.

Kolmogorov, A. N. The local structure of turbulence in incompressible viscous fluid for very large Reynolds numbers. Dokl. Akad. Nauk SSSR. 299-303.

Kwon, S. J. \& Seo, I. W. 2005. Reynolds number effects on the behavior of a nonbuoyant round jet. Experiments in Fluids, 38, 801-812.

Lai, J. C. S. \& Nasr, A. 1998. Two parallel plane jets: comparison of the performance of three turbulence models. Proceedings of the Institution of Mechanical Engineers Part G-Journal of Aerospace Engineering, 212, 379-391.

Larraona, G. S., Rivas, A., Anton, R., Ramos, J. C., Pastor, I. \& Moshfegh, B. 2013. Computational parametric study of an impinging jet in a cross-flow configuration for electronics cooling applications. Applied Thermal Engineering, 52, 428-438.

Launder, B. E. \& Spalding, D. B. 1972. Mathematical Models of Turbulence, Academic Press.

Launder, B. E. \& Spalding, D. B. 1974. The numerical computation of turbulent flows. Computer Methods in Applied Mechanics and Engineering, 3, 269-289.

Launder, B. E. 1989a. Second-moment closure and its use in modelling turbulent industrial flows. International Journal for Numerical Methods in Fluids, 9, 963985.

Launder, B. E. 1989b. Second-moment closure: present... and future? International Journal of Heat and Fluid Flow, 10, 282-300.

Launder, B. E. \& Sandham, N. D. 2002. Closure Strategies for Turbulent and Transitional Flows, Cambridge University Press.

Lien, F. S. \& Leschziner, M. A. 1994. Assessment of turbulence-transport models including non-linear rng eddy-viscosity formulation and second-moment closure for flow over a backward-facing step. Computers and Fluids, 23, 983-1004.

Lipari, G. \& Stansby, P. K. 2011. Review of Experimental Data on Incompressible Turbulent Round Jets. Flow Turbulence and Combustion, 87, 79-114. 
Magi, V., Iyer, V. \& Abraham, J. 2001. The k- $\varepsilon$ model and computed spreading rates in round and plane jets. Numerical Heat Transfer; Part A: Applications, 40, 317334.

Malmström, T. G., Kirkpatrick, A. T., Christensen, B. \& Knappmiller, K. D. 1997. Centreline velocity decay measurements in low-velocity axisymmetric jets. Journal of Fluid Mechanics, 346, 363-377.

Marsters, G. F. 1979. Measurements in the Flowfield of a Linear Array of Rectangular Nozzles. Journal of Aircraft, 17, 774-780.

Meslem, A., Nastase, I. \& Allard, F. 2010. Passive mixing control for innovative air diffusion terminal devices for buildings. Building and Environment, 45, 26792688 .

Mi, J., Nathan, G. J. \& Nobes, D. S. 2001. Mixing characteristics of axisymmetric free jets from a contoured nozzle, an orifice plate and a pipe. Journal of Fluids Engineering-Transactions of the ASME, 123, 878-883.

Miller, D. R. \& Comings, E. W. 1960. Force-momentum fields in a dual-jet flow. Journal of Fluid Mechanics, 7, 237-256.

Montgomery, D. C. 2012. Design and Analysis of Experiments, 8th Edition, John Wiley \& Sons.

Morel, T. 1975. Comprehensive Design of Axisymmetric Wind Tunnel Contractions. Journal of Fluids Engineering, Transactions of the ASME, 97 Ser 1, 225-233.

Murai, K., Taga, M. \& Akagawa, K. 1976. Experimental study on confluence of two twodimensional jets. Bull JSME, 19, 958-964.

Nasr, A. \& Lai, J. C. S. 1997. Two parallel plane jets: mean flow and effects of acoustic excitation. Experiments in Fluids, 22, 251-260.

Nasr, A. \& Lai, J. C. S. 2000. The Effects of Nozzle Spacing on the Development of Two Parallel Plane Jets. International Journal of Transport Phenomena, 2, 57-70.

Norton, T., Grant, J., Fallon, R. \& Sun, D. W. 2010. Optimising the ventilation configuration of naturally ventilated livestock buildings for improved indoor environmental homogeneity. Building and Environment, 45, 983-995.

Okamoto, T., Yagita, M., Watanabe, A. \& Kawamura, K. 1985. Interaction of Twin Turbulent Circular Jet. Bulletin of the JSME-Japan Society of Mechanical Engineers, 28, 617-622.

Olsson, M. \& Fuchs, L. 1996. Large eddy simulation of the proximal region of a spatially developing circular jet. Physics of Fluids, 8, 2125-2137.

Pani, B. \& Dash, R. 1983. Three Dimensional Single and Multiple Free Jets. Journal of Hydraulic Engineering, 109, 254-269.

Patankar, S. V. \& Spalding, D. B. 1972. A calculation procedure for heat, mass and momentum transfer in three-dimensional parabolic flows. International Journal of Heat and Mass Transfer, 15, 1787-1806.

Pope, S. B. 1978. An explanation of the turbulent round-jet/plane-jet anomaly. AIAA Journal, 16, 279-281.

Quinn, W. R. 2006. Upstream nozzle shaping effects on near field flow in round turbulent free jets. European Journal of Mechanics, B/Fluids, 25, 279-301.

Raffel, M., Willert, C. E., Wereley, S. T. \& Kompenhans, J. 2007. Particle Image Velocimetry: A Practical Guide, Springer.

Rajaratnam, N. 1976. Turbulent Jets.

Reynolds, O. 1883. An Experimental Investigation of the Circumstances Which Determine Whether the Motion of Water Shall Be Direct or Sinuous, and of the Law of Resistance in Parallel Channels. Philosophical Transactions of the Royal Society of London, 174, 935-982. 
Richardson, L. F. 1922. Weather Prediction by Numerical Process, by Lewis Richardson, University Press.

Ricou, F. P. \& Spalding, D. B. 1961. Measurements of entrainment by axisymmetrical turbulent jets. Journal of Fluid Mechanics, 11, 21-32.

Rieth, M., Proch, F., Stein, O. T., Pettit, M. W. A. \& Kempf, A. M. 2014. Comparison of the Sigma and Smagorinsky LES Models for Grid Generated Turbulence and a Channel Flow. Computers \& Fluids.

Roache, P. J. 1997. Quantification of uncertainty in computational fluid dynamics. Annual Review of Fluid Mechanics, 29, 123.

Rokni, M. \& Sundén, B. 1999a. Turbulence modeling experience in ducts with forced convection flow. Numerical Heat Transfer; Part A: Applications, 35, 629-654.

Rokni, M. \& Sundén, B. 1999b. Performance of RNG Turbulence Modelling for Forced Convective Heat Transfer in Ducts. International Journal of Computational Fluid Dynamics, 11, 351-362.

Smith, L. M. \& Woodruff, S. L. 1998. Renormalization-group analysis of turbulence. Annual Review of Fluid Mechanics.

Soloff, S. M., Adrian, R. J. \& Liu, Z. C. 1997. Distortion compensation for generalized stereoscopic particle image velocimetry. Measurement Science and Technology, 8, 1441-1454.

Speziale, C. G. \& Thangam, S. 1992. Analysis of an RNG based turbulence model for separated flows. International Journal of Engineering Science, 30, 1379-IN4.

Stein, O. T., Böhm, B., Dreizler, A. \& Kempf, A. M. 2011. Highly-resolved LES and PIV analysis of isothermal turbulent opposed jets for combustion applications. Flow, Turbulence and Combustion, 87, 425-447.

Stravent, O. STRAVENT S11 - Universaldon för stratifierande ventilation.

Tanaka, E. 1970. The interference of two-dimensional parallel jets, 1. Bull JSME, 13, 272-280.

Tatsumi, K., Tanaka, M., Woodfield, P. L. \& Nakabe, K. 2010. Swirl and buoyancy effects on mixing performance of baffle-plate-type miniature confined multijet. International Journal of Heat and Fluid Flow, 31, 45-56.

Thielen, L., Jonker, H. J. J. \& Hanjalic, K. 2003. Symmetry breaking of flow and heat transfer in multiple impinging jets. International Journal of Heat and Fluid Flow, 24, 444-453.

Thielen, L., Hanjalić, K., Jonker, H. \& Manceau, R. 2005. Predictions of flow and heat transfer in multiple impinging jets with an elliptic-blending second-moment closure. International Journal of Heat and Mass Transfer, 48, 1583-1598.

Todde, V., Spazzini, P. G. \& Sandberg, M. 2009. Experimental analysis of low-Reynolds number free jets: Evolution along the jet centerline and Reynolds number effects. Experiments in Fluids, 47, 279-294.

Tu, J., Yeoh, G. H. \& Liu, C. 2012. Computational Fluid Dynamics: A Practical Approach, Elsevier Science.

Tummers, M. J. 1999. Investigation of a turbulent wake in an adverse pressure gradient using laser Doppler anemometry.

Wang, L., Hejcik, J. \& Sunden, B. 2007. PIV measurement of separated flow in a square channel with streamwise periodic ribs on one wall. Journal of Fluids Engineering, Transactions of the ASME, 129, 834-841.

Wang, L., Salewski, M. \& Sundén, B. 2010. Turbulent flow in a ribbed channel: Flow structures in the vicinity of a rib. Experimental Thermal and Fluid Science, 34, $165-176$. 
Versteeg, H. K. \& Malalasekera, W. 2007. An Introduction to Computational Fluid Dynamics: The Finite Volume Method, Pearson Education.

Westerweel, J. 1994. Efficient detection of spurious vectors in particle image velocimetry data. Experiments in Fluids, 16, 236-247.

Westerweel, J. \& Scarano, F. 2005. Universal outlier detection for PIV data. Experiments in Fluids, 39, 1096-1100.

Wilcox, D. C. 1994. Turbulence Modeling for CFD, DCW Industries.

Villermaux, E. \& Hopfinger, E. J. 1994. Periodically arranged co-flowing jets. Journal of Fluid Mechanics, 263, 63-92.

Wolfshtein, M. 1969. The velocity and temperature distribution in one-dimensional flow with turbulence augmentation and pressure gradient. International Journal of Heat and Mass Transfer, 12, 301-318.

Vouros, A. \& Panidis, T. 2008. Influence of a secondary, parallel, low Reynolds number, round jet on a turbulent axisymmetric jet. Experimental Thermal and Fluid Science, 32, 1455-1467.

Wygnanski, I. \& Fiedler, H. 1969. Some measurements in the self-preserving jet. Journal of Fluid Mechanics, 38, 577-612.

Yakhot, V. \& Orszag, S. A. 1986. Renormalization-Group Analysis of Turbulence. Physical Review Letters, 57, 1722-1724.

Yakhot, V. \& Smith, L. M. 1992. The renormalization group, the e \{open\}-expansion and derivation of turbulence models. Journal of Scientific Computing, 7, 35-61.

Yakhot, V., Orszag, S. A., Thangam, S., Gatski, T. B. \& Speziale, C. G. 1992. Development of Turbulence Models for Shear Flows by a Double Expansion Technique. Physics of Fluids a-Fluid Dynamics, 4, 1510-1520.

Yimer, I., Becker, H. A. \& Grandmaison, E. W. 1996. Development of Flow from Multiple-Jet Burners. Canadian Journal of Chemical Engineering, 74, 840-851.

Yin, Z. Q., Zhang, H. J. \& Lin, J. Z. 2007. Experimental Study on the Flow Field Characteristics in the Mixing Region of Twin Jets. Journal of Hydrodynamics, 19, 309-313. 